\title{
UNIT GROUPS OF MAXIMAL ORDERS IN TOTALLY DEFINITE QUATERNION ALGEBRAS OVER REAL QUADRATIC FIELDS
}

\author{
QUN LI, JIANGWEI XUE, AND CHIA-FU YU
}

\begin{abstract}
We study a form of refined class number formula (resp. type number formula) for maximal orders in totally definite quaternion algebras over real quadratic fields, by taking into consideration the automorphism groups of right ideal classes (resp. unit groups of maximal orders). For each finite noncyclic group $G$, we give an explicit formula for the number of conjugacy classes of maximal orders whose unit groups modulo center are isomorphic to $G$, and write down a representative for each conjugacy class. This leads to a complete recipe (even explicit formulas in special cases) for the refined class number formula for all finite groups. As an application, we prove the existence of superspecial abelian surfaces whose endomorphism algebras coincide with $\mathbb{Q}(\sqrt{p})$ in all positive characteristic $p \not \equiv 1(\bmod 24)$.
\end{abstract}

\section{Contents}

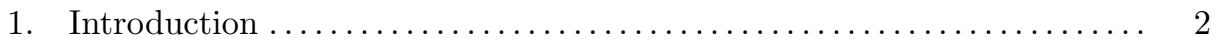

2. Preliminaries on orders in quaternion algebras $\ldots \ldots \ldots \ldots \ldots \ldots \ldots \ldots, 6$

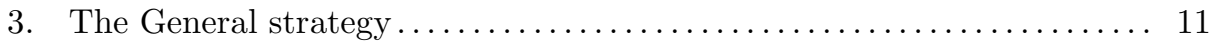

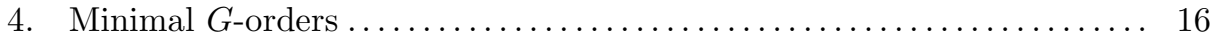

4.1. Vignéras unit index of $\mathcal{O}$ and the fundamental unit of $F=\mathbb{Q}(\sqrt{d})$. 16

4.2. Structure of the reduced unit group of a CM-extension $K / F \ldots \ldots 17$

4.3. Finite noncyclic subgroups of $H^{\times} / O_{F}^{\times} \ldots \ldots \ldots \ldots \ldots \ldots \ldots \ldots \ldots, 18$

4.4. Structure of the minimal $G$-orders for noncyclic finite groups $G \ldots 22$

4.5. Normalizers of minimal orders ....................... 24

5. Refined type numbers for noncyclic reduced unit groups: part I...... 25

6. Refined type numbers for noncyclic reduced unit groups: part II ..... 31

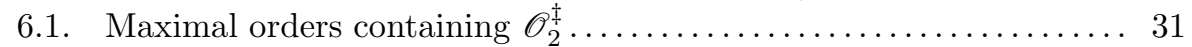

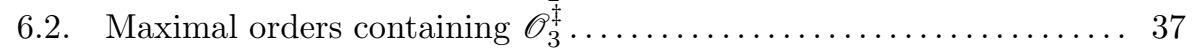

7. Quadratic $O_{F}$-orders in CM-fields ........................... 41

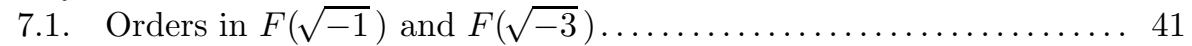

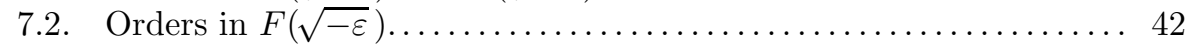

8. Calculations for $F=\mathbb{Q}(\sqrt{p})$ and $H=H_{\infty_{1}, \infty_{2}} \ldots \ldots \ldots \ldots \ldots \ldots \ldots$

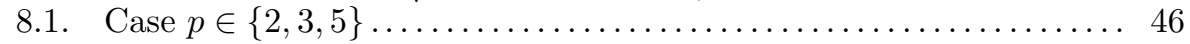

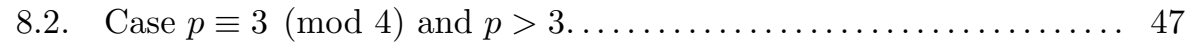

9. Superspecial abelian surfaces $\ldots \ldots \ldots \ldots \ldots \ldots \ldots \ldots \ldots \ldots \ldots \ldots, 49$

Date: June 4, 2019.

2010 Mathematics Subject Classification. 11R52, 11R29, 11G10.

Key words and phrases. quaternion orders, unit groups, superspecial abelian surfaces, class number formula. 
9.1. The first application. ............. 50

9.2. Pop's result on embedding problems $\ldots \ldots \ldots \ldots \ldots \ldots \ldots \ldots \ldots \ldots$

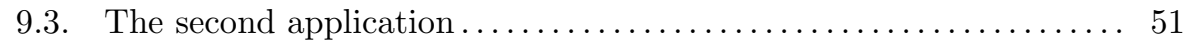

Acknowledgements . . . . . . . . . . . .

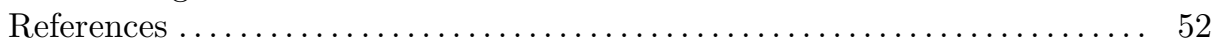

\section{INTRODUCTION}

Let $F$ be a totally real number field with ring of integers $O_{F}$, and $H$ be a totally definite quaternion $F$-algebra, that is, $H \otimes_{F, \sigma} \mathbb{R}$ is isomorphic to the Hamilton quaternion $\mathbb{R}$-algebra $\mathbb{H}$ for every embedding $\sigma: F \hookrightarrow \mathbb{R}$. Fix a maximal $O_{F}$-order $\mathbb{O}$ in $H$. The class number $h(\mathbb{O})$, by definition, is the cardinality of the finite set $\mathrm{Cl}(\mathbb{O})$ of right ideal classes of $\mathbb{O}$. It depends only on $H$ and is also denoted by $h(H)$ and called the class number of $H$. The type number $t(H)$ is the cardinality of the finite set $\operatorname{Tp}(H)$ of all $H^{\times}$-conjugacy classes of maximal $O_{F}$-orders of $H$.

For any $O_{F}$-order $\mathcal{O}$ in $H$, the quotient group $\mathcal{O}^{\star}:=\mathcal{O}^{\times} / O_{F}^{\times}$of the unit group $\mathcal{O}^{\times}$by $O_{F}^{\times}$is finite by [40, Theorem V.1.2] and called the reduced unit group of $\mathcal{O}$. For a (fractional) right ideal $I \subset H$ of $\mathcal{O}$, the reduced automorphism group of $I$ is defined to be $\mathcal{O}_{l}(I)^{\star}$, where $\mathcal{O}_{l}(I):=\{x \in H \mid x I \subseteq I\}$ is the left order of $I$. The reduced unit group $\mathcal{O}^{\star}$ can easily be regarded as a finite subgroup of $\mathrm{SO}_{3}(\mathbb{R}$ ) (see Section 3.1). By the well-known classification of finite subgroups of $\mathrm{SO}_{3}(\mathbb{R}), \mathcal{O}^{\star}$ is isomorphic to either a cyclic group $C_{n}$ of order $n$, a dihedral group $D_{n}$ of order $2 n$, or one of the groups $A_{4}, S_{4}$ and $A_{5}$ (see [40, Theorem I.3.6]). For each group $G$ in this list, we define

$$
\begin{aligned}
\operatorname{Cl}(\mathbb{O}, G) & :=\left\{[I] \in \operatorname{Cl}(\mathbb{O}) \mid \mathcal{O}_{l}(I)^{\star} \simeq G\right\}, & h(H, G): & =|\operatorname{Cl}(\mathbb{O}, G)|, \\
\operatorname{Tp}(H, G) & :=\left\{\llbracket \mathbb{O}^{\prime} \rrbracket \in \operatorname{Tp}(H) \mid \mathbb{O}^{\prime \star} \simeq G\right\}, & t(H, G): & =|\operatorname{Tp}(H, G)|,
\end{aligned}
$$

where $[I]$ denotes the right ideal class of $I$, and $\llbracket \mathbb{O}^{\prime} \rrbracket$ denotes the $H^{\times}$-conjugacy class of the maximal order $\mathbb{O}^{\prime}$. The quantity $h(H, G)$ (resp. $t(H, G)$ ) can be regarded as a refined class number (resp. refined type number) of $H$. The classical proof of the independence of $h(H)$ on the choice of the maximal order $\mathbb{O}$ can be adapted to show that the same holds for $h(H, G)$ as well (see [40, Lemma I.4.9(2)]). Indeed, any two maximal orders $\mathbb{O}, \mathbb{O}^{\prime}$ are linked, that is to say, there exists a full $O_{F}$-lattice $J \subset H$ such that $\mathcal{O}_{l}(J)=\mathbb{O}$ and $\mathcal{O}_{r}(J)=\mathbb{O}^{\prime}$, where $\mathcal{O}_{r}(J):=\{x \in H \mid J x \subseteq J\}$. The map $[I] \mapsto[I J]$ defines a bijection $\mathrm{Cl}(\mathbb{O}) \rightarrow \mathrm{Cl}\left(\mathbb{O}^{\prime}\right)$. Since $\mathcal{O}_{l}(I)=\mathcal{O}_{l}(I \bar{J})$ for each $I$, it follows from the definition that $h(H, G)$ does not depend on the choice of $\mathbb{O}$. If $H$ is clear from the context, then we drop it from the notation and write $h(G)$ and $t(G)$ instead.

The main tool for studying class numbers and type numbers is Eichler's trace formula ( 15,29 , cf. [40]). This has been used to study various arithmetic problems concerning totally definite quaternion algebras, including the analogous Gauss problem and the cancellation property by several people [8, 17, 21, 22, 35, 39]. Brzezinski [8] obtains a complete list of all orders (including non-Gorenstein orders) in definite quaternion $\mathbb{Q}$-algebras with class number one. Kirschmer and Voight 22 determine all Eichler $O_{F}$-orders with class number $\leq 2$. Kirschmer and Lorch 21$]$ determine all Eichler $O_{F}$-orders with type number $\leq 2$. 
Vignéras [38, Theorem 3.1] gives an explicit formula for $h(\mathbb{O})$ (including Eichler orders $(\mathbb{O})$ when $F$ is a real quadratic field. Explicit formulas for type numbers, however, are comparably unknown. In [30] Pizer proves a general formula for type numbers, and uses this to deduce an explicit type number formula for Eichler orders in an arbitrary definite quaternion $\mathbb{Q}$-algebra 30 . So far there is no known explicit formula for $t(H)$ in the literature when $F$ is an arbitrary quadratic field. As far as the authors are aware, the only known cases are due to Kitaoka 23, 1.12 and 3.11] and Ponomarev [31, Theorem part (c), p. 102] when $F=\mathbb{Q}(\sqrt{p})$ with a prime number $p$, and $H=H_{\infty_{1}, \infty_{2}}$ is the totally definite quaternion $F$-algebra unramified at every finite place of $F$. Under the same hypothesis on $H$, we prove the following result for more general totally real fields in [48, Section 3]. The idea of the proof is sketched in Remark 3.6 for the convenience of the readers.

Proposition 1.1. Let $H$ be a totally definite quaternion algebra over a totally real number field $F$ of even degree over $\mathbb{Q}$. Assume that $H$ is unramified at all finite places of $F$ and that $h(F)$ is odd. Then for any finite group $G$ one has $h(H, G)=h(F) t(H, G)$. In particular, the equality $h(H)=h(F) t(H)$ holds.

Thanks to Vignéras's explicit formula [38, Theorem 3.1], we obtain an explicit formula for $t\left(H_{\infty_{1}, \infty_{2}}\right)$ when $F$ has odd class number. For a complete list of quadratic fields with odd class numbers, see [11, Corollary 18.4]. In particular, $h(\mathbb{Q}(\sqrt{p}))$ is odd for every prime $p$.

The task of this paper is to determine explicitly the refined class number $h(H, G)$ for an arbitrary totally definite $H$ over any real quadratic field $F$. A key step turns out to be determining explicitly $t(H, G)$ for all finite noncyclic $G$. Let $d \in \mathbb{N}$ be the square-free positive integer such that $F=\mathbb{Q}(\sqrt{d})$, and $\varepsilon \in O_{F}^{\times}$be the fundamental unit of $F$. The cases $d \in\{2,3,5\}$ will be treated separately. Suppose that $d \geq 6$. Up to isomorphism, the reduced unit group $\mathcal{O}^{\star}$ of any $O_{F}$-order $\mathcal{O} \subset H$ falls into the following list as shown in Section 4 .

$$
\mathcal{G}=\left\{C_{1}, C_{2}, C_{3}, C_{4}, C_{6}, D_{2}^{\dagger}, D_{2}^{\ddagger}, D_{3}^{\dagger}, D_{3}^{\ddagger}, D_{4}, D_{6}, A_{4}, S_{4}\right\} .
$$

Recall that $C_{n}$ denotes the cyclic group of order $n$, and $D_{n}$ denotes the dihedral group of order $2 n$, so $D_{2}$ is just the Klein 4-group. The reduced unit groups isomorphic to $D_{n}$ for $n=2,3$ are further distinguished into two different kinds (labeled by $D_{n}^{\dagger}$ and $D_{n}^{\ddagger}$ respectively) according to the reduced norms of certain units (see Definition 4.3.3). The cases $D_{n}^{\ddagger}$ with $n \in\{2,3\}$ occur only when $\mathrm{N}_{F / \mathbb{Q}}(\varepsilon)=1$ since the reduced norm of every nonzero element in a totally definite quaternion algebra is totally positive. For a noncyclic group $G$ in $\mathcal{G}$, we introduce in Definitions 4.3 .1 and 4.4 .1 the notion of minimal $G$-orders. Essentially, a minimal $G$-order is an

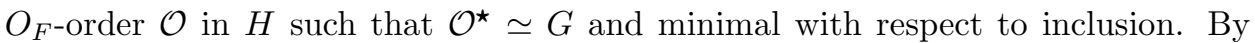
Proposition 4.3.5, a minimal $G$-order, if exists, is unique up to $O_{F}$-isomorphism. Since any maximal $O_{F}$-order $\mathbb{O}$ with $\mathbb{O}^{\star} \supseteq G$ contains a minimal $G$-order, the calculation of $t(H, G)$ reduces to counting and classifying the maximal orders containing a fixed minimal $G$-order $\mathcal{O}$. Let $\beth(\mathcal{O})$ be the number of conjugacy classes of maximal orders containing $\mathcal{O}$.

We summarize our main results as the following two theorems. See Section 5 and Section 6 . 
Theorem 1.2. Suppose that $d \geq 6$. We have

$$
\begin{aligned}
t\left(D_{6}\right) & = \begin{cases}1 & \text { if } H \simeq\left(\frac{-1,-3}{F}\right) \text { and } 3 \varepsilon \in F^{\times 2} ; \\
0 & \text { otherwise; }\end{cases} \\
t\left(S_{4}\right)=t\left(D_{4}\right) & = \begin{cases}1 & \text { if } H \simeq\left(\frac{-1,-1}{F}\right) \text { and } 2 \varepsilon \in F^{\times 2} ; \\
0 & \text { otherwise; }\end{cases} \\
t\left(A_{4}\right) & = \begin{cases}1 & \text { if } H \simeq\left(\frac{-1,-1}{F}\right) \text { and } 2 \varepsilon \notin F^{\times 2} ; \\
0 & \text { otherwise; }\end{cases} \\
t\left(D_{2}^{\dagger}\right) & = \begin{cases}1 & \text { if } H \simeq\left(\frac{-1,-1}{F}\right),\left(\frac{F}{2}\right)=0 \text { and } 2 \varepsilon \notin F^{\times 2} ; \\
0 & \text { otherwise; }\end{cases} \\
t\left(D_{3}^{\dagger}\right) & = \begin{cases}1 & \text { if } H \simeq\left(\frac{-1,-3}{F}\right) \text { and } 3 \varepsilon \notin F^{\times 2} ; \\
0 & \text { otherwise. }\end{cases}
\end{aligned}
$$

Here the Artin symbol $\left(\frac{F}{2}\right)=0$ if and only if 2 is ramified in $F$.

Theorem 1.3. Suppose that $d \geq 6$. For $n \in\{2,3\}$, let $\mathscr{O}_{n}^{\ddagger}$ be a minimal $D_{n}^{\ddagger}$-order, which is unique up to $O_{F}$-isomorphism if exists.

$$
\text { If } \mathrm{N}_{F / \mathbb{Q}}(\varepsilon)=1 \text { and } H \simeq\left(\frac{-1,-\varepsilon}{F}\right) \text {, then }
$$

$$
t\left(D_{2}^{\ddagger}\right)+t\left(D_{4}\right)+t\left(S_{4}\right)+t\left(D_{6}\right)=\beth\left(\mathscr{O}_{2}^{\ddagger}\right) .
$$

If $\mathrm{N}_{F / \mathbb{Q}}(\varepsilon)=1$ and $H \simeq\left(\frac{-\varepsilon,-3}{F}\right)$, then

$$
t\left(D_{3}^{\ddagger}\right)+t\left(S_{4}\right)+t\left(D_{6}\right)=\beth\left(\mathscr{O}_{3}^{\ddagger}\right) .
$$

The numbers $\beth\left(\mathscr{O}_{n}^{\ddagger}\right)$ for $n=2,3$ are determined explicitly in Propositions 6.1.2 and 6.2.2. In all the remaining cases, minimal $D_{n}^{\ddagger}$-orders do not exist and $t\left(D_{n}^{\ddagger}\right)=0$.

Moreover, for each finite noncyclic group $G \in \mathcal{G}$, if a maximal $G$-order (maximal $O_{F}$-order with reduced unit group $G$ ) exists, then we write down explicitly a representative $\mathbb{O}^{\prime}$ in each $H^{\times}$-conjugacy class and calculate its normalizer $\mathcal{N}\left(\mathbb{O}^{\prime}\right)$. This leads to explicit formulas of $h(G)$ for noncyclic groups $G \in \mathcal{G}$ in Section 5 and Section 6.

The strategy for computing $h\left(C_{n}\right)$ with $n>1$ is described in Section 3.7. The main idea is to apply the trace formula (3.4) (see [40, Theorem III.5.2]). According to (3.19), the computations depend on the classification of maximal orders with noncyclic reduced unit groups. Lastly, $h\left(C_{1}\right)$ is computed using the mass formula (3.23) and the knowledge of $h(G)$ for every nontrivial group $G$. In conclusion, we obtain the following result:

Let $F=\mathbb{Q}(\sqrt{d})$ be a real quadratic field and $H$ be an arbitrary totally definite quaternion $F$-algebra. We have a complete recipe for writing down $h(G)$ for each finite group $G$.

In fact, the only obstacle between us and a complete formula for $h(G)$ is the overwhelming number of cases that the problem naturally divides into, rendering any unified formula too cumbersome and unwieldy. However, for any class of quadratic real fields that one has a good grasp on the fundamental units, the deduction of explicit formulas for $h(G)$ based on our recipe becomes entirely routine. One such 
example is when $H=H_{\infty_{1}, \infty_{2}}$ and $d=p$ is a prime; see Theorems 1.5 and 1.6. This extends a result of Hashimoto 18 by a different method.

Remark 1.4. We emphasize that same as Vigéras's explicit formula 38, Theorem 3.1], our refined formula depends not only on the square-free integer $d$ defining $F$ but also on a good understanding of the fundamental unit $\varepsilon \in O_{F}^{\times}$. Our recipe for $h(G)$ refines the explicit formula for $h(H)$ given by Vignéras, which in turn is an application of the more general Eichler's class number formula (3.25) 40, Corollaire V.2.5]. However, the equality $h(H)=\sum_{G \in \mathcal{G}} h(G)$ does not provide an alternative approach to her formula. Indeed, to compute $h\left(C_{n}\right)$ with $n \in\{1,2,3,4,6\}$, we have to work out all the information required by Eichler's formula, so summing all $h(G)$ together merely reproduces Vignéras's result along the same route. See Section 3.8 for more details.

For the following two theorems, let $p \in \mathbb{N}$ be a prime, $F=\mathbb{Q}(\sqrt{p})$, and $H=$ $H_{\infty_{1}, \infty_{2}}$ be the totally definite quaternion $F$-algebra unramified at all the finite places of $F$. We write $\zeta_{F}(s)$ for the Dedekind zeta function of $F$, whose special value at $s=-1$ can be calculated using Siegel's formula [49, Table 2, p. 70]:

$$
\zeta_{F}(-1)=\frac{1}{60} \sum_{\substack{b^{2}+4 a c=\mathfrak{d}_{F} \\ a, c>0}} a,
$$

where $\mathfrak{d}_{F}$ denotes the discriminant of $F$, and $a, b, c \in \mathbb{Z}$. For simplicity, denote the class number $h(\mathbb{Q}(\sqrt{m}))$ by $h(m)$ for any square-free $m \in \mathbb{Z}$. We first recall a result of Hashimoto [18].

Theorem 1.5 (Hashimoto). If $p \equiv 1(\bmod 4)$ and $p>5$, then

$$
\begin{aligned}
& t\left(C_{1}\right)=\frac{\zeta_{F}(-1)}{2}-\frac{h(-p)}{8}-\frac{h(-3 p)}{12}-\frac{1}{4}\left(\frac{p}{3}\right)-\frac{1}{4}\left(\frac{2}{p}\right)+\frac{1}{2} \\
& t\left(C_{2}\right)=\frac{h(-p)}{4}+\frac{1}{2}\left(\frac{p}{3}\right)+\frac{1}{4}\left(\frac{2}{p}\right)-\frac{3}{4} \\
& t\left(C_{3}\right)=\frac{h(-3 p)}{4}+\frac{1}{4}\left(\frac{p}{3}\right)+\frac{1}{2}\left(\frac{2}{p}\right)-\frac{3}{4} \\
& t\left(D_{3}\right)=\frac{1}{2}\left(1-\left(\frac{p}{3}\right)\right) \\
& t\left(A_{4}\right)=\frac{1}{2}\left(1-\left(\frac{2}{p}\right)\right) .
\end{aligned}
$$

We get the results for the remaining primes $p$ as a direct application of our recipe.

Theorem 1.6. If $p \equiv 3(\bmod 4)$ and $p>5$, then

$$
\begin{aligned}
& t\left(C_{1}\right)=\frac{\zeta_{F}(-1)}{2}+\left(-7+3\left(\frac{2}{p}\right)\right) \frac{h(-p)}{8}-\frac{h(-2 p)}{4}-\frac{h(-3 p)}{12}+\frac{3}{2}, \\
& t\left(C_{2}\right)=\left(2-\left(\frac{2}{p}\right)\right) \frac{h(-p)}{2}+\frac{h(-2 p)}{2}-\frac{5}{2}, \\
& t\left(C_{3}\right)=\frac{h(-3 p)}{4}-1, \\
& t\left(C_{4}\right)=\left(3-\left(\frac{2}{p}\right)\right) \frac{h(-p)}{2}-1,
\end{aligned}
$$




$$
\begin{aligned}
& t\left(D_{3}\right)=1, \\
& t\left(D_{4}\right)=1, \\
& t\left(S_{4}\right)=1 .
\end{aligned}
$$

For $p=2,3$ and 5 , we have

\begin{tabular}{|c|c|c|c|}
\hline$p$ & 2 & 3 & 5 \\
\hline$t(H)$ & 1 & 2 & 1 \\
\hline$t(G)$ & $t\left(S_{4}\right)=1$ & $t\left(S_{4}\right)=t\left(D_{12}\right)=1$ & $t\left(A_{5}\right)=1$ \\
\hline
\end{tabular}

Lastly, we apply the above results to the study of superspecial abelian surfaces [24, Definition 1.7, Ch.1]. Indeed, one of our motivations is to count the number of certain superspecial abelian surfaces with a fixed reduced automorphism group $G$. This extends results of our earlier works [44,45,46,47] where we compute explicitly the number of these abelian surfaces over finite fields. We also construct superspecial abelian surfaces $X$ over some field $K$ of characteristic $p$ with endomorphism algebra $\operatorname{End}^{0}(X)=\mathbb{Q}(\sqrt{p})$, provided that $p \not \equiv 1(\bmod 24)$. The construction makes use of results of Florian Pop [32] on embedding problems for large fields.

This paper is organized as follows. In Section 2, we recall some preliminary results on orders in quaternion algebras. The general strategy for computing $h(G)$ for totally definite quaternion algebras over arbitrary totally real fields is explained in Section 3. We restrict ourselves to the case of quadratic real fields $F=\mathbb{Q}(\sqrt{d})$ starting from Section 4 where we introduce the concept of minimal $G$-orders for finite noncyclic groups $G$. Section 5 and Section 6 contain the case-by-case study of the maximal orders containing the minimal $G$-orders for each $G$, and the results are summarized in Theorems 1.2 and 1.3 respectively. In Section 7 , we classify the quadratic $O_{F}$-orders with nontrivial reduced unit groups in CM-extensions of $F$. The formulas in Theorem 1.6 are calculated in Section 8 . We conclude with two applications to superspecial abelian surfaces in Section 9 .

\section{Preliminaries on orders in QUaternion algebras}

Throughout this section, $F$ is either a global field or a non-archimedean local field, and $H$ is a quaternion $F$-algebra. The algebra $H$ admits a canonical involution $x \mapsto \bar{x}$ such that $\operatorname{Tr}(x)=x+\bar{x}$ and $\operatorname{Nr}(x)=x \bar{x}$ are respectively the reduced trace and reduced norm of $x \in H$. We always assume that $\operatorname{char}(F) \neq 2$. If $H=\left(\frac{a, b}{F}\right)$ for $a, b \in F^{\times}$, then $\{1, i, j, k\}$ denotes the standard $F$-basis of $H$ subjected to the following multiplication rules

$$
k=i j, \quad i^{2}=a, \quad j^{2}=b, \quad \text { and } \quad i j=-j i .
$$

When $F$ is local, $\left(\frac{a, b}{F}\right)$ splits over $F$ if and only if the Hilbert symbol $(a, b)=1$. Often, $H$ is also presented as $K+K x$, where $K$ is a separable $F$-algebra of dimension 2 , and $x \in H$ is an element such that

$$
x^{2}=c \in F^{\times}, \quad \text { and } \quad \forall y \in K, x y=\bar{y} x .
$$

Here $y \mapsto \bar{y}$ is the unique nontrivial $F$-automorphism of $K$. Following [40, Section I.1], we write $H=\{K, c\}$ for the above presentation.

If $F$ is local, then we fix a uniformizer $\pi \in F^{\times}$, and write $\nu: F^{\times} \rightarrow \mathbb{Z}$ for the discrete valuation of $F$. Denote by $O_{F}, \mathfrak{p}, \mathfrak{k}$ respectively the valuation ring, the maximal ideal and the residue field of $\nu$. If $F$ is global, we fix a finite set $S$ of places 
of $F$ containing all the archimedean ones, and write $O_{F}$ for the ring of $S$-integers of $F$. In fact, when $F$ is a number field, $S$ will always be the set of archimedean valuations (unless specified otherwise), so $O_{F}$ is just the usual ring of integers of $F$. For a nonzero prime ideal $\mathfrak{p} \subset O_{F}$, its corresponding discrete valuation is denoted by $\nu_{\mathfrak{p}}$, and the $\mathfrak{p}$-adic completion of $F$ is denoted by $F_{\mathfrak{p}}$.

Let $\Lambda$ be an $O_{F}$-lattice in $H$, i.e. a finitely generated $O_{F}$-submodule that spans $H$ over $F$. Its dual lattice is defined to be $\Lambda^{\vee}:=\left\{x \in H \mid \operatorname{Tr}(x \Lambda) \subseteq O_{F}\right\}$. An order in $H$ always refers to an $O_{F}$-lattice that is at the same time a subring of $H$ containing $O_{F}$. For an order $\mathcal{O} \subset H$, any maximal order $\mathbb{O}$ containing $\mathcal{O}$ is a lattice intermediate to $\mathcal{O} \subseteq \mathcal{O}^{\vee}$. There are only finitely many such lattices.

The discriminant of an order $\mathcal{O} \subset H$ is denoted by $\mathfrak{d}(\mathcal{O})$. If $\mathcal{O}$ is a free $O_{F^{-}}$ module with basis $\left\{x_{1}, \ldots, x_{4}\right\}$ (e.g. when $F$ is local), then $\mathfrak{d}(\mathcal{O})$ is the square root of the $O_{F}$-ideal $\operatorname{det}\left(\operatorname{Tr}\left(x_{s} \bar{x}_{t}\right)_{1 \leq s, t \leq 4}\right) O_{F}$. If $\mathcal{O}^{\prime} \subseteq \mathcal{O}$ is a suborder of $\mathcal{O}$, then $\mathfrak{d}\left(\mathcal{O}^{\prime}\right)=\chi\left(\mathcal{O}, \mathcal{O}^{\prime}\right) \mathfrak{d}(\mathcal{O})$, where $\chi\left(\mathcal{O}, \mathcal{O}^{\prime}\right)$ is the ideal index of $\mathcal{O}^{\prime} \subseteq \mathcal{O}$ as in 34, Section III.1]. For any finite extension $K / F$, we have $\mathfrak{d}\left(\mathcal{O} \otimes_{O_{F}} O_{K}\right)=\mathfrak{d}(\mathcal{O}) O_{K}$. An order $\mathcal{O}$ is maximal if and only if $\mathfrak{d}(\mathcal{O})$ coincides with the discriminant $\mathfrak{d}(H)$ of $H$, defined as the product of all finite primes of $F$ that are ramified in $H$ [25, Definition 2.7.4]. When $F$ is global, $\mathcal{O}_{\mathfrak{p}}$ denotes the $\mathfrak{p}$-adic completion of $\mathcal{O}$ at a finite prime $\mathfrak{p}$.

2.1. Let $\mathcal{N}(\mathcal{O})=\left\{x \in H^{\times} \mid x \mathcal{O} x^{-1}=\mathcal{O}\right\}$ be the normalizer of $\mathcal{O}$. First suppose that $F$ is local. Following [7, Section 2], we say that an element $x \in H^{\times}$is even (resp. odd) if $\nu(\operatorname{Nr}(x))$ is even (resp. odd). The notion of parity applies to elements of $H^{\times} / F^{\times}$as well. Clearly, any unit $u \in \mathcal{O}^{\times}$is even. Let $\mathbb{O}$ be a maximal order in a split quaternion algebra $H \simeq M_{2}(F)$. Then $\mathbb{O}$ is $H^{\times}$-conjugate to $M_{2}\left(O_{F}\right)$, and $\mathcal{N}(\mathbb{O})=F^{\times} \mathbb{O}^{\times}$by [40, Section II.2, p. 40] (see also [7, Proposition 2.1]). In particular, if $x$ is odd, then $x \notin \mathcal{N}(\mathbb{O})$.

If $F$ is global and $\mathfrak{p}$ is a finite prime of $F$, an element $x \in H^{\times}$is said to be even (resp. odd) at $\mathfrak{p}$ if $\nu_{\mathfrak{p}}(\mathrm{Nr}(x))$ is even (resp. odd).

Lemma 2.2. Let $\mathbb{O}$ be a maximal order in $H$, and $u \in \mathcal{N}(\mathbb{O})$ be an element of the normalizer of $\mathbb{O}$. If $\operatorname{Nr}(u) \in O_{F}$, then $u \in \mathbb{O}$; if further $\operatorname{Nr}(u) \in O_{F}^{\times}$, then $u \in \mathbb{O}^{\times}$.

Proof. Suppose that $\operatorname{Nr}(u) \in O_{F}$. We show that $u \in \mathbb{O}_{\mathfrak{p}}$ for every finite prime $\mathfrak{p}$ of $F$. If $H$ is ramified at $\mathfrak{p}$, then $\mathbb{O}_{\mathfrak{p}}$ coincides with the unique maximal order $\{z \in$ $\left.H_{\mathfrak{p}} \mid \operatorname{Nr}(z) \in O_{F_{\mathfrak{p}}}\right\}$ in $H_{\mathfrak{p}}$; if $H$ splits at $\mathfrak{p}$, then $u \in \mathbb{O}_{\mathfrak{p}}$ because $\mathcal{N}\left(\mathbb{O}_{\mathfrak{p}}\right)=F_{\mathfrak{p}}^{\times} \mathbb{O}_{\mathfrak{p}}^{\times}$ as explained above. The second part of the lemma follows immediately.

2.3. Given an order $\mathcal{O}$ in $H$, we write $\mathfrak{S}(\mathcal{O})$ for the set of maximal orders containing $\mathcal{O}$, and $\aleph(\mathcal{O})$ for the cardinality of $\mathfrak{S}(\mathcal{O})$. The quotient group $\mathcal{N}(\mathcal{O}) / \mathcal{O}^{\times}$acts naturally on $\mathfrak{S}(\mathcal{O})$ by conjugation. If $F$ is global and $\mathfrak{p}$ is a nonzero prime ideal of $O_{F}$, we set $\aleph_{\mathfrak{p}}(\mathcal{O}):=\aleph\left(\mathcal{O}_{\mathfrak{p}}\right)$. Note that $\mathcal{O}_{\mathfrak{p}}$ is maximal (forcing $\aleph_{\mathfrak{p}}(\mathcal{O})=1$ ) for almost all $\mathfrak{p}$, and

$$
\aleph(\mathcal{O})=\prod_{\mathfrak{p}} \aleph_{\mathfrak{p}}(\mathcal{O}),
$$

where the product runs over all finite primes of $F$. If further $F$ is a number field and $p \in \mathbb{N}$ is an integral prime, then we set $\aleph_{p}(\mathcal{O})=\prod_{\mathfrak{p} \mid\left(p O_{F}\right)} \aleph_{\mathfrak{p}}(\mathcal{O})$.

Suppose that $F$ is local. If $H$ is division, then it has a unique maximal order, so $\aleph(\mathcal{O})=1$ for all $\mathcal{O}$. Now suppose that $H=M_{2}(F)$. The Bruhat-Tits tree $\mathfrak{T}$ (of $\left.\mathrm{PGL}_{2}(F)\right)$ is a homogeneous tree of degree $|\mathfrak{k}|+1$ whose vertices are the maximal 
orders of $M_{2}(F)$ and such that two vertices are connected by an edge if and only if the two maximal orders have distance one [40, Section II.2]. Let $\mathfrak{T}(\mathcal{O})$ be the subtree whose set of vertices is $\mathfrak{S}(\mathcal{O})$. For example, if $\mathcal{O}=\mathbb{O} \cap \mathbb{O}^{\prime}$ is an Eichler order of level $\pi^{n} O_{F}$, i.e. the intersection of two maximal orders $\mathbb{O}$ and $\mathbb{O}^{\prime}$ of distance $n$, then by [6, Corollary 2.5], $\mathfrak{T}(\mathcal{O})$ is the unique path connecting $\mathbb{O}$ and $\mathbb{O}^{\prime}$ on $\mathfrak{T}$, and $\aleph(\mathcal{O})=n+1$. In general, Arenas-Carmona [3] has shown that $\mathfrak{T}(\mathcal{O})$ is a thick line, i.e. the maximal subtree whose vertices lie no further than a fixed distance (called the depth) from a line segment, which is called the stem of the thick line. The stem may have length 0 , in which case it degenerates into a single vertex. A thick line with stem length 0 and depth 1 is called a star, and its stem is called the center of the star. Arenas-Carmona and Saavedra 4 provide concrete formulas for the depth and stem length (and hence $\aleph(\mathcal{O})$ ) of $\mathfrak{T}(\mathcal{O})$ when $\mathcal{O}$ is generated by a pair of orthogonal pure quaternions. However, the present paper does not depend on their formulas. It is also worthwhile to mention Fang-Ting Tu's result [37 that the intersection of any finite number of maximal orders coincides with the intersection of three maximal orders.

2.4. Let $\mathfrak{J}(\mathcal{O})$ be the Jacobson radical of $\mathcal{O}$, and $\mathfrak{k}^{\prime} / \mathfrak{k}$ be the unique quadratic extension of the finite field $\mathfrak{k}$. First, suppose that $F$ is local. Using lifting of idempotents, one shows that $\mathcal{O} \simeq M_{2}\left(O_{F}\right)$ if and only if $\mathcal{O} / \mathfrak{J}(\mathcal{O}) \simeq M_{2}(\mathfrak{k})$ (cf. 6, Proposition 2.1]). When $\mathcal{O} \nsucceq M_{2}\left(O_{F}\right)$, we have

$$
\mathcal{O} / \mathfrak{J}(\mathcal{O}) \simeq \mathfrak{k}, \quad \mathfrak{k} \times \mathfrak{k}, \quad \text { or } \quad \mathfrak{k}^{\prime},
$$

and the Eichler invariant $e(\mathcal{O})$ is defined to be 0,1 or -1 accordingly [6, Definition 1.8]. The Eichler invariant of $M_{2}\left(O_{F}\right)$ is defined to be 2. By [14, Chapter 6, Exercise 14], $e(\mathcal{O})$ behaves under a finite field extension $K / F$ in the following way: if $e(\mathcal{O})=-1$ and $\mathfrak{k}^{\prime}$ is contained in the residue field of $K$, then $e\left(\mathcal{O} \otimes_{O_{F}} O_{K}\right)=1$, otherwise $e\left(\mathcal{O} \otimes_{O_{F}} O_{K}\right)=e(\mathcal{O})$. We refer to [6, Section 1] for the concepts of Gorenstein, Bass, and hereditary orders. If $e(\mathcal{O})=1$, then $\mathcal{O}$ is an Eichler order and hence a Bass order. If $e(\mathcal{O})=-1$, then $\mathcal{O}$ is Bass as well. The Bass orders are explicitly described in [7, Section 1]. The orders with Eichler invariant 0 are more complicated, and the Gorenstein ones are discussed in [6, Section 4].

If $F$ is global and $\mathfrak{p}$ is a nonzero prime ideal of $O_{F}$, we denote by $e_{\mathfrak{p}}(\mathcal{O})$ the Eichler invariant of $\mathcal{O}_{\mathfrak{p}}$. An order $\mathcal{O}$ is Gorenstein (resp. Bass, resp. hereditary) if and only if $\mathcal{O}_{\mathfrak{p}}$ is Gorenstein (resp. Bass, resp. hereditary) for every $\mathfrak{p}$.

The Brandt invariant $\mathfrak{b}(\mathcal{O})$ is defined to be $\mathfrak{d}(\mathcal{O}) \operatorname{Nr}\left(\mathcal{O}^{\vee}\right)$. By [6, Proposition 1.3], $\mathfrak{b}(\mathcal{O})$ is an integral ideal of $O_{F}$, and $\mathcal{O}$ is Gorenstein if and only if $\mathfrak{b}(\mathcal{O})=O_{F}$.

Lemma 2.5. Let $H \simeq M_{2}(F)$ be a split quaternion algebra over a local field $F$, and $\mathcal{O} \subseteq H$ be a Bass order with Eichler invariant $e(\mathcal{O})=0$. Then $\aleph(\mathcal{O})=2$.

Proof. By 6, Corollary 4.3] and 7, Section 1], every maximal order containing $\mathcal{O}$ necessarily contains its hereditary closure $\mathcal{H}(\mathcal{O})$, which has discriminant $\pi O_{F}$. Since $H$ splits over $F, \mathcal{H}(\mathcal{O})$ is an Eichler order of level $\pi O_{F}$. There are precisely two maximal orders containing it.

Lemma 2.6. Let $F$ be a local field, and $H=M_{2}(F)$. Up to $H^{\times}$-conjugation, $\mathcal{O}=O_{F}+\pi M_{2}\left(O_{F}\right)$ is the unique non-Gorenstein order with $\mathfrak{d}(\mathcal{O})=\pi^{3} O_{F}$. The subtree $\mathfrak{T}(\mathcal{O})$ is a star centered at the Gorenstein saturation ${ }^{1} M_{2}\left(O_{F}\right)$ of $\mathcal{O}$. In particular, $\aleph(\mathcal{O})=|\mathfrak{k}|+2$.

\footnotetext{
${ }^{1}$ It is also called the Gorenstein closure in some literature.
} 
Proof. First, suppose that $\mathcal{O}=O_{F}+\pi M_{2}\left(O_{F}\right)$. Its discriminant is

$$
\mathfrak{d}(\mathcal{O})=\chi\left(M_{2}\left(O_{F}\right), \mathcal{O}\right) \mathfrak{d}\left(M_{2}\left(O_{F}\right)\right)=\pi^{3} O_{F} .
$$

The dual lattice of $\mathcal{O}$ is

$$
\mathcal{O}^{\vee}=\frac{1}{\pi}\left\{\left[\begin{array}{ll}
a & b \\
c & d
\end{array}\right] \in M_{2}\left(O_{F}\right) \mid a \equiv d \quad\left(\bmod \pi O_{F}\right)\right\},
$$

which has reduced norm $\operatorname{Nr}\left(\mathcal{O}^{\vee}\right)=\frac{1}{\pi^{2}} O_{F}$. Hence the Brandt invariant $\mathfrak{b}(\mathcal{O})$ is $\mathfrak{d}(\mathcal{O}) \operatorname{Nr}\left(\mathcal{O}^{\vee}\right)=\pi O_{F}$. By the criterion in [ $\underline{6}$, Proposition 1.3], $\mathcal{O}$ is non-Gorenstein. In the notation of [4, Section 1.1], $\mathcal{O}=M_{2}\left(O_{F}\right)^{[1]}$, so $\mathfrak{T}(\mathcal{O})$ is a star centered at $M_{2}\left(O_{F}\right)$. More concretely, a maximal order contains $\mathcal{O}=O_{F}+\pi M_{2}\left(O_{F}\right)$ if and only if it has at most distance 1 from $M_{2}\left(O_{F}\right)$. The star has a unique center and $1+|\mathfrak{k}|$ external vertices, so $\aleph(\mathcal{O})=|\mathfrak{k}|+2$.

Conversely, let $\mathcal{O}$ be an arbitrary non-Gorenstein order in $H=M_{2}(F)$ with $\mathfrak{d}(\mathcal{O})=\pi^{3} O_{F}$, and $\operatorname{Gor}(\mathcal{O})$ be its Gorenstein saturation. By [6, Propositon 1.4],

$$
\mathcal{O}=O_{F}+\mathfrak{b}(\mathcal{O}) \operatorname{Gor}(\mathcal{O}), \quad \text { where } \quad \mathfrak{b}(\mathcal{O})=\pi^{r} O_{F} \quad \text { with } \quad r>1 .
$$

Hence $\mathfrak{d}(\operatorname{Gor}(\mathcal{O}))=\chi(\operatorname{Gor}(\mathcal{O}), \mathcal{O})^{-1} \mathfrak{d}(\mathcal{O})=\mathfrak{b}(\mathcal{O})^{-3} \mathfrak{d}(\mathcal{O})=\pi^{3-3 r} O_{F}$. Since $\mathfrak{d}(\operatorname{Gor}(\mathcal{O}))$ is integral, $r=1$ and $\operatorname{Gor}(\mathcal{O})$ is a maximal order in $M_{2}(F)$. Thus $\mathcal{O}$ is conjugate to $O_{F}+\pi M_{2}\left(O_{F}\right)$.

Lemma 2.7. Let $H=\left(\frac{a, b}{F}\right)$ with $a, b \in O_{F} \backslash\{0\}$. The order $\mathcal{O}=O_{F}[i, j]$ is a Gorenstein order with discriminant $\mathfrak{d}(\mathcal{O})=4 a b O_{F}$. Suppose further that $F$ is a number field, and both $a, b \in O_{F}^{\times}$. Then $\mathcal{O}$ is maximal at every nondyadic prime of $F$, and $e_{\mathfrak{p}}(\mathcal{O})=0$ for every dyadic prime $\mathfrak{p}$. Moreover, if $a=-1$, then $(1+i) \in \mathcal{N}(\mathcal{O})$

Proof. One calculates that $\mathfrak{d}(\mathcal{O})=4 a b O_{F}$, and the dual basis of $\{1, i, j, k\}$ is

$$
\left\{\frac{1}{2}, \quad \frac{i}{2 a}, \quad \frac{j}{2 b}, \frac{-k}{2 a b}\right\} .
$$

In particular, $O_{F}=\mathfrak{d}(\mathcal{O}) \operatorname{Nr}(-k / 2 a b) \subseteq \mathfrak{d}(\mathcal{O}) \operatorname{Nr}\left(\mathcal{O}^{\vee}\right)=\mathfrak{b}(\mathcal{O})$. It follows from 6 , Proposition 1.3] that $\mathcal{O}$ is Gorenstein. Suppose that $F$ is a number field and $a, b \in O_{F}^{\times}$. We have $\mathfrak{d}(\mathcal{O})=4 O_{F}$, and the maximality of $\mathcal{O}$ at the nondyadic primes of $F$ follows directly. Let $\mathfrak{p}$ be a dyadic prime of $F$ with finite residue field $\mathfrak{k}$. Then $\mathcal{O}_{\mathfrak{p}}$ is not maximal since $\mathfrak{d}\left(\mathcal{O}_{\mathfrak{p}}\right)$ is not square-free. By (2.4), an equation of the form $x^{2}=c \in \mathfrak{k}$ with $x \in \mathcal{O}_{\mathfrak{p}} / \mathfrak{J}\left(\mathcal{O}_{\mathfrak{p}}\right)$ has a unique solution that lies in $\mathfrak{k}$. It follows that the reductions of both $i$ and $j$ modulo $\mathfrak{J}\left(\mathcal{O}_{\mathfrak{p}}\right)$ are in $\mathfrak{k}$, and hence $\mathcal{O}_{\mathfrak{p}} / \mathfrak{J}\left(\mathcal{O}_{\mathfrak{p}}\right)=\mathfrak{k}$, i.e. $e_{\mathfrak{p}}(\mathcal{O})=0$. When $a=-1$, we have

$$
(1+i) i(1+i)^{-1}=i \quad \text { and } \quad(1+i) j(1+i)^{-1}=k .
$$

Thus $(1+i) \in \mathcal{N}(\mathcal{O})$.

Lemma 2.8. Let $H=\left(\frac{a, b}{F}\right)$ with $a, b \in O_{F} \backslash\{0\}$, and $\mathcal{O}=O_{F}[i, j]$. Suppose that $F$ is a local field, $\mathfrak{d}(\mathcal{O})=4 a b O_{F}$ is divisible by $\pi^{3} O_{F}$, and $e(\mathcal{O})=0$. Then $\mathcal{O}$ is a Bass order if and only if at least one of the orders $O_{F}[i]$ or $O_{F}[j]$ is maximal in its total quotient ring.

Proof. The sufficiency follows directly from [7, Proposition 1.11]. We prove the converse. So assume that neither $O_{F}[i]$ nor $O_{F}[j]$ are maximal orders. Then there 
exists a unique overorder $B \subset F(i)$ of $O_{F}[i]$ such that $\chi\left(B, O_{F}[i]\right)=\pi O_{F}$. Similarly, we find an overorder $B^{\prime} \subset F(j)$ of $O_{F}[j]$ with $\chi\left(B^{\prime}, O_{F}[j]\right)=\pi O_{F}$. Now let $O=B+B j$, and $O^{\prime}=B^{\prime}+i B^{\prime}$. These are distinct overorders of $\mathcal{O}$ since $O \cap F(i)=B$ while $O^{\prime} \cap F(i)=O_{F}[i] \neq B$. The choices of $B$ and $B^{\prime}$ imply that

$$
\chi(O, \mathcal{O})=\chi\left(O^{\prime}, \mathcal{O}\right)=\pi^{2} O_{F} .
$$

Hence $\mathfrak{d}(O)=\mathfrak{d}\left(O^{\prime}\right)=\pi^{-2} \mathfrak{d}(\mathcal{O}) \neq O_{F}$ by the assumption on $\mathfrak{d}(\mathcal{O})$. It follows from [6. Corollary 4.3] that $\mathcal{O}$ cannot be Bass, otherwise the overorder of $\mathcal{O}$ satisfying (2.6) is unique.

Lemma 2.9. Let $H=\left(\frac{a, b}{F}\right)$ with $a, b \in O_{F} \backslash\{0\}$ and $b \equiv 1\left(\bmod 4 O_{F}\right)$. Then $\mathcal{O}:=O_{F}[i,(1+j) / 2]$ is a Gorenstein order with $\mathfrak{d}(\mathcal{O})=a b O_{F}$ and $j \in \mathcal{N}(\mathcal{O})$. Suppose further that $F$ is a number field, $a \in O_{F}^{\times}$and $b=-3$. Then $\mathcal{O}$ is maximal at any nonzero prime of $O_{F}$ coprime to 3 . Let $\mathfrak{p}$ be a finite prime of $F$ above 3 with residue field $\mathfrak{k}$. If $H_{\mathfrak{p}}$ is division and $\mathfrak{p}$ is unramified above 3 , then $\mathcal{O}_{\mathfrak{p}}$ is maximal; otherwise we have

$$
e_{\mathfrak{p}}(\mathcal{O})= \begin{cases}1 & \text { if }(a \bmod \mathfrak{p}) \in\left(\mathfrak{k}^{\times}\right)^{2}, \\ -1 & \text { if }(a \bmod \mathfrak{p}) \notin\left(\mathfrak{k}^{\times}\right)^{2} .\end{cases}
$$

Proof. Note that $(1+j) / 2$ is integral over $O_{F}$ since $b \equiv 1\left(\bmod 4 O_{F}\right)$, and one easily verifies that $j \in \mathcal{N}(\mathcal{O})$. The order $\mathcal{O}$ is a free $O_{F}$-module with basis $\{1, i,(1+$ $j) / 2,(i+k) / 2\}$. Direct calculation shows that the dual basis is

$$
\left\{\frac{b-j}{2 b}, \frac{b i+k}{2 a b}, \frac{j}{b}, \frac{k}{a b}\right\}
$$

and $\mathfrak{d}(\mathcal{O})=a b O_{F}$. By [6, Proposition 1.3(b)], $\mathcal{O}$ is Gorenstein since $\mathfrak{b}(\mathcal{O})=O_{F}$.

Now suppose that $F$ is a number field, $a \in O_{F}^{\times}$and $b=-3$. Then $\mathfrak{d}(\mathcal{O})=3 O_{F}$, and hence $\mathcal{O}$ is maximal at any nonzero prime of $O_{F}$ coprime to 3 . If $\mathfrak{p}$ is a prime unramified above 3 and $H_{\mathfrak{p}}$ is division, then $\mathfrak{d}\left(\mathcal{O}_{\mathfrak{p}}\right)=\mathfrak{p} O_{F_{\mathfrak{p}}}=\mathfrak{d}\left(H_{\mathfrak{p}}\right)$, so $\mathcal{O}_{\mathfrak{p}}$ is the unique maximal order in $H_{\mathfrak{p}}$. In the remaining cases, $\mathcal{O}_{\mathfrak{p}}$ is non-maximal. Since $(1+j) / 2$ is a primitive third root of unity and $\operatorname{char}(\mathfrak{k})=3$, we have $(1+j) / 2 \equiv 1$ $\left(\bmod \mathfrak{J}\left(\mathcal{O}_{\mathfrak{p}}\right)\right)$ by (2.4). Let $\breve{\imath}$ denote the image of $i$ in $\mathcal{O}_{\mathfrak{p}} / \mathfrak{J}\left(\mathcal{O}_{\mathfrak{p}}\right)$. We then have

$$
\mathfrak{k}[\breve{\imath}]= \begin{cases}\mathfrak{k} \oplus \mathfrak{k} & \text { if } \quad(a \bmod \mathfrak{p}) \in\left(\mathfrak{k}^{\times}\right)^{2}, \\ \mathfrak{k}^{\prime} & \text { if } \quad(a \bmod \mathfrak{p}) \notin\left(\mathfrak{k}^{\times}\right)^{2} .\end{cases}
$$

The formula for $e_{\mathfrak{p}}(\mathcal{O})$ follows.

For the convenience of the reader, we state the following well-known lemma.

Lemma 2.10. Let $Q$ be a non-archimedean local field with ring of integers $R$ and uniformizer $\pi_{0}$, and $H_{0}$ be a quaternion division algebra over $Q$ with the unique maximal $R$-order $\mathbb{O}_{0}$. Suppose that $F / Q$ is an unramified quadratic extension. Then $\mathcal{O}:=\mathbb{O}_{0} \otimes_{R} O_{F}$ is an Eichler order of level $\pi_{0} O_{F}$ in $H:=H_{0} \otimes_{Q} F \simeq M_{2}(F)$.

Proof. As remarked in Section 2.4 $\mathcal{O}$ is an order with Eichler invariant 1 and discriminant $\pi_{0} O_{F}$. The lemma follows from [6, Proposition 2.1].

We treat the case where $F / Q$ is ramified next. 
Lemma 2.11. Let $Q, R, H_{0}, \mathbb{O}_{0}$ and $\pi_{0}$ be as in Lemma 2.10, Suppose that $F / Q$ is a ramified quadratic extension. Then there is a unique $O_{F}$-order $\mathbb{O}$ in $H:=$ $H_{0} \otimes_{Q} F \simeq M_{2}(F)$ properly containing $\mathcal{O}:=\mathbb{O}_{0} \otimes_{R} O_{F}$. The order $\mathbb{O}$ is necessarily maximal, and $\mathcal{O} \simeq O_{L}+\pi \mathbb{O}$, where $L / F$ is the unique unramified quadratic extension of $F$, identified with a subfield of $H$ such that $O_{L} \subset \mathbb{O}$.

By [40, Theoreme 3.2], any two embeddings of $O_{L}$ into $\mathbb{O}$ are conjugate by an element of $\mathbb{O}^{\times}$. Thus the structure of $O_{L}+\pi \mathbb{O}$ does not depend on the choice of the embedding.

Proof. By Section [2.4 $e(\mathcal{O})=e\left(\mathbb{O}_{0}\right)=-1$. So according to [6, Proposition 3.1], there is a unique minimal overorder $\mathbb{O}$ containing $\mathcal{O}$ with $\chi(\mathbb{O}, \mathcal{O})=\pi^{2} O_{F}$ and $\mathfrak{J}(\mathcal{O})=\pi \mathbb{O}$. We have

$$
\mathfrak{d}(\mathbb{O})=\mathfrak{d}\left(\mathbb{O}_{0}\right) \chi(\mathbb{O}, \mathcal{O})^{-1}=\pi_{0} \pi^{-2} O_{F}=O_{F} .
$$

Therefore, $\mathbb{O}$ is a maximal order in $H$, and hence the unique order properly containing $\mathcal{O}$.

Let $L_{0} / Q$ be the unique unramified quadratic extension of $Q$, identified with a subfield of $H_{0}$ such that $H_{0}=L_{0}+L_{0} x$ with $x^{2}=\pi_{0}$ and $x y=\bar{y} x$ for all $y \in L_{0}$. Then by [40, Corollary II.1.7], $\mathbb{O}_{0}=O_{L_{0}}+O_{L_{0}} x$. Since $F / Q$ is ramified, we have $L=L_{0} \otimes_{\mathbb{Q}} F$ and $O_{L}=O_{L_{0}} \otimes_{R} O_{F}$. Hence

$$
\mathcal{O}=\mathbb{O}_{0} \otimes_{R} O_{F}=O_{L}+O_{L} x .
$$

In particular, $\mathcal{O} \supseteq O_{L}+\pi \mathbb{O}$. On the other hand, $\chi\left(\mathbb{O}, O_{L}+\pi \mathbb{O}\right)=\pi^{2} O_{F}=\chi(\mathbb{O}, \mathcal{O})$. Thus $\mathcal{O}=O_{L}+\pi \mathbb{O}$ (see also [7, Proposition 1.12]).

\section{The General strategy}

In this section, we assume that $F$ is a totally real number field, and $H$ is a totally definite quaternion $F$-algebra. We keep the notation in the introduction and fill in the details for the strategy of computing $h(G)=h(H, G)$ in (1.1). Let $\mathcal{O}$ be an arbitrary $O_{F}$-order in $H$. We first study the general structure of the reduced unit group $\mathcal{O}^{\star}=\mathcal{O}^{\times} / O_{F}^{\times}$.

3.1. Fix an embedding $\sigma: F \hookrightarrow \mathbb{R}$. We have the following successive inclusions of groups

$$
\mathcal{O}^{\star}=\mathcal{O}^{\times} / O_{F}^{\times} \hookrightarrow H^{\times} / F^{\times} \stackrel{\sigma}{\hookrightarrow} \mathbb{H}^{\times} / \mathbb{R}^{\times} .
$$

The quotient $\mathbb{H}^{\times} / \mathbb{R}^{\times}$is isomorphic to the special orthogonal group $\mathrm{SO}_{3}(\mathbb{R})$, whose finite subgroups have been classified [40, Section I.3]. Thus $\mathcal{O}^{\star}$ is isomorphic to one of the following groups:

- a cyclic group $C_{n}$ of order $n \geq 1$;

- a dihedral group $D_{n}$ of order $2 n$ with $n \geq 2$;

- an exceptional group $A_{4}, S_{4}$, or $A_{5}$.

Since $H$ is totally definite, the field $F(x)$ generated by a non-central element $x \in H$ is a CM-extension (i.e. a totally imaginary quadratic extension) of $F$. An $O_{F^{-}}$ order in a CM-extension of $F$ is called a $C M O_{F}$-order. If $x \in \mathcal{O}$, then $B:=F(x) \cap \mathcal{O}$ is a CM $O_{F}$-order; if further $x \in \mathcal{O}^{\times}$, then

$$
w(B):=\left[B^{\times}: O_{F}^{\times}\right]>1
$$

since $x \in \mathcal{O}^{\times}$is non-central. Let $\mathscr{B}$ be the set of all CM $O_{F}$-orders $B$ (up to isomorphism) with $w(B)>1$. We recall the following two facts: 
(i) $\mathscr{B}$ is a finite set;

(ii) the reduced unit group $B^{\star}:=B^{\times} / O_{F}^{\times}$is cyclic for every $B \in \mathscr{B}$.

Indeed, Pizer denotes the set $\mathscr{B}$ by " $C_{1}$ " in [30, Remarks, p. 92] shows that it is a subset of a larger finite set " $C$ " (ibid.). Classically, the finiteness of $\mathscr{B}$ guarantees that the summation in the Eichler's class number formula (3.25) has only finitely many terms. When $F$ is a quadratic real field $\mathbb{Q}(\sqrt{d})$ with square-free $d \geq 6$, the set $\mathscr{B}$ is classified in Section 7 . We will prove (ii) in Lemma 4.2.1] (see also [45, Section 2.1]).

3.2. Let $\mathcal{O}$ be an arbitrary $O_{F}$-order in $H$. Given an $O_{F}$-order $B$ inside a CMextension $K / F$, we write $\operatorname{Emb}(B, \mathcal{O})$ for the finite set of optimal $O_{F}$-embeddings of $B$ into $\mathcal{O}$. In other words,

$$
\operatorname{Emb}(B, \mathcal{O}):=\left\{\varphi \in \operatorname{Hom}_{F}(K, H) \mid \varphi(K) \cap \mathcal{O}=\varphi(B)\right\} .
$$

The unit group $\mathcal{O}^{\times}$acts on $\operatorname{Emb}(B, \mathcal{O})$ from the right by $\varphi \mapsto u^{-1} \varphi u$ for all $\varphi \in \operatorname{Emb}(B, \mathcal{O})$ and $u \in \mathcal{O}^{\times}$, and the action descends to $\mathcal{O}^{\star}$. We denote

$$
m\left(B, \mathcal{O}, \mathcal{O}^{\times}\right):=\left|\operatorname{Emb}(B, \mathcal{O}) / \mathcal{O}^{\times}\right| .
$$

For each finite prime $\mathfrak{p}$ of $F$, we set

$$
m_{\mathfrak{p}}(B):=m\left(B_{\mathfrak{p}}, \mathcal{O}_{\mathfrak{p}}, \mathcal{O}_{\mathfrak{p}}^{\times}\right)=\left|\operatorname{Emb}\left(B_{\mathfrak{p}}, \mathcal{O}_{\mathfrak{p}}\right) / \mathcal{O}_{\mathfrak{p}}^{\times}\right|
$$

Given a fractional locally principal right ideal $I$ of $\mathcal{O}$, we write $[I]$ for its ideal class, and $\mathcal{O}_{l}(I)$ for its associated left order $\{x \in H \mid x I \subseteq I\}$. Let $\mathrm{Cl}(\mathcal{O})$ be the finite set of locally principal right ideal classes of $\mathcal{O}$. By [40, Theorem 5.11, p. 92],

$$
\sum_{[I] \in \mathrm{Cl}(\mathcal{O})} m\left(B, \mathcal{O}_{l}(I), \mathcal{O}_{l}(I)^{\times}\right)=h(B) \prod_{\mathfrak{p}} m_{\mathfrak{p}}(B),
$$

where the product on the right runs over all finite primes of $F$, and $m_{\mathfrak{p}}(B)=1$ for all but finitely many $\mathfrak{p}$. A priori, [40, Theorem 5.11] is stated for Eichler orders, but it applies in much more general settings. See [42, Lemma 3.2] and [44, Lemma 3.2.1]. When $\mathcal{O}$ is maximal, we have

$$
m_{\mathfrak{p}}(B):= \begin{cases}1-\left(\frac{B}{\mathfrak{p}}\right) & \text { if } \mathfrak{p} \mid \mathfrak{d}(H) \\ 1 & \text { otherwise }\end{cases}
$$

where $\left(\frac{B}{\mathfrak{p}}\right)$ is the Eichler symbol [40, p. 94 and Section II.3].

3.3. Suppose that $\mathcal{O}^{\times} \neq O_{F}^{\times}$. We explain the basic idea of counting $m\left(B, \mathcal{O}, \mathcal{O}^{\times}\right)$ for $B \in \mathscr{B}$. A nontrivial cyclic subgroup of $\mathcal{O}^{\star}$ is said to be maximal if it is not properly contained in any other cyclic subgroup of $\mathcal{O}^{\star}$. For each $\mathrm{CM} O_{F}$-order $B \in \mathscr{B}$, an optimal $O_{F^{-}}$-embedding $\varphi: B \rightarrow \mathcal{O}$ identifies $B^{\star}$ with a maximal cyclic subgroup of $\mathcal{O}^{\star}$. Conversely, let $C$ be a maximal cyclic subgroup of $\mathcal{O}^{\star}$, and $u \in \mathcal{O}^{\times}$ be a representative of an arbitrary nontrivial element of $C$. The CM-field $F(u) \subset H$ depends only on $C$ and not on the choice of $u$, and the same holds true for the $O_{F^{-}}$ order $B_{C}:=F(u) \cap \mathcal{O}$. There is an optimal $O_{F}$-embedding $\varphi: B \rightarrow \mathcal{O}$ such that $\varphi\left(B^{\star}\right)=C$ if and only if $B \simeq B_{C}$.

For each $B \in \mathscr{B}$, let $\mathscr{C}(B, \mathcal{O})$ be the subset of maximal cyclic subgroups $C \subseteq \mathcal{O}^{\star}$ such that $B_{C} \simeq B$. It is clear that $\mathscr{C}(B, \mathcal{O})$ is invariant under the conjugation by $\mathcal{O}^{\star}$ from the right. We have an $\mathcal{O}^{\star}$-equivariant surjective $2: 1$ map

$$
\operatorname{Emb}(B, \mathcal{O}) \rightarrow \mathscr{C}(B, \mathcal{O}), \quad \varphi, \bar{\varphi} \mapsto \varphi\left(B^{\star}\right),
$$


where $\bar{\varphi}$ denotes the complex conjugate of $\varphi$.

For each $C \in \mathscr{C}(B, \mathcal{O})$, let $N_{\mathcal{O}^{\star}}(C)$ be the normalizer of $C$ in $\mathcal{O}^{\star}$. We claim that the induced map

$$
\operatorname{Emb}(B, \mathcal{O}) / \mathcal{O}^{\star} \rightarrow \mathscr{C}(B, \mathcal{O}) / \mathcal{O}^{\star}
$$

is a 2:1 cover ramified over the conjugacy classes $[C]$ with $N_{\mathcal{O}^{\star}}(C) \neq C$. In other words, $\varphi$ and $\bar{\varphi}$ belong to the same $\mathcal{O}^{\times}$-conjugacy class if and only if $N_{\mathcal{O}^{\star}}\left(\varphi\left(B^{\star}\right)\right) \neq$ $\varphi\left(B^{\star}\right)$. The necessity is obvious. For the sufficiency, suppose that $\tilde{v} \in N_{\mathcal{O}^{\star}}\left(\varphi\left(B^{\star}\right)\right)$ and $\tilde{v} \notin \varphi\left(B^{\star}\right)$. Let $v \in \mathcal{O}^{\times}$be a representative of $\tilde{v}$. Then $v^{-1} \varphi(B) v=\varphi(B)$ since $\varphi(B)$ is uniquely determined by the maximal cyclic subgroup $\varphi\left(B^{\star}\right) \subseteq \mathcal{O}^{\star}$. It follows that $v^{-1} \varphi v \in\{\varphi, \bar{\varphi}\}$. But $v^{-1} \varphi v \neq \varphi$, otherwise $v$ lies in the centralizer of $\varphi(B)$ in $\mathcal{O}$, which is $\varphi(B)$ itself. Therefore, $v^{-1} \varphi v=\bar{\varphi}$, and our claim is verified.

The dihedral group $D_{2}$ is just the Klein 4-group. It is abelian and has 3 maximal cyclic subgroups of order 2 . If $\mathcal{O}^{\star} \simeq D_{2}$, then (3.6) is a bijection and $m\left(B, \mathcal{O}, \mathcal{O}^{\times}\right)=$ $\left|\mathscr{C}(B, \mathcal{O}) / \mathcal{O}^{\star}\right|=|\mathscr{C}(B, \mathcal{O})|$.

Proposition 3.4. Assume that $\mathcal{O}^{\times} \neq O_{F}^{\times}$and $\mathcal{O}^{\star} \nsucceq D_{2}$. Then $m\left(B, \mathcal{O}, \mathcal{O}^{\times}\right) \leq 2$ for every $C M O_{F}$-order $B \in \mathscr{B}$. More explicitly, we have the following cases.

(1) If $B \nsucc B_{C}$ for any maximal cyclic subgroup $C$ of $\mathcal{O}^{\star}$, then $\operatorname{Emb}(B, \mathcal{O})=\emptyset$, and hence $m\left(B, \mathcal{O}, \mathcal{O}^{\times}\right)=0$.

(2) If $\mathcal{O}^{\star}$ is cyclic, then there is a unique $B^{\prime} \in \mathscr{B}$ such that $\operatorname{Emb}\left(B^{\prime}, \mathcal{O}\right) \neq \emptyset$. Moreover, we have

$$
m\left(B^{\prime}, \mathcal{O}, \mathcal{O}^{\times}\right)=2 .
$$

Assume further that $B \simeq B_{C}$ for some maximal cyclic subgroup $C$ of $\mathcal{O}^{\star}$.

(3) Suppose that $\mathcal{O}^{\star} \simeq D_{n}$ with $n \geq 3$. If $n$ is even, then $\mathcal{O}^{\star}$ has two conjugacy classes of maximal cyclic subgroups of order 2 , denoted by $\left[C^{\prime}\right]$ and $\left[C^{\prime \prime}\right]$.

$$
m\left(B, \mathcal{O}, \mathcal{O}^{\times}\right)= \begin{cases}1 & \text { if }|C|=n ; \\ 2 & \text { if }|C|=2 \text { and } n \text { is odd; } \\ 1 & \text { if }|C|=2, n \text { is even and } B_{C^{\prime}} \nsucc B_{C^{\prime \prime}} ; \\ 2 & \text { if }|C|=2, n \text { is even and } B_{C^{\prime}} \simeq B_{C^{\prime \prime}} .\end{cases}
$$

(4) If $\mathcal{O}^{\star} \simeq S_{4}$ or $A_{5}$, then $m\left(B, \mathcal{O}, \mathcal{O}^{\times}\right)=1$; if $\mathcal{O}^{\star} \simeq A_{4}$, then

$$
m\left(B, \mathcal{O}, \mathcal{O}^{\times}\right)= \begin{cases}1 & \text { if }|C|=2 ; \\ 2 & \text { if }|C|=3 .\end{cases}
$$

Proof. Part (1) follows directly from Section 3.3 . The remaining parts all reduce to counting the conjugacy classes of maximal cyclic subgroups in $\mathcal{O}^{\star}$ and working out the normalizers. For example, if $\mathcal{O}^{\star} \simeq D_{n}$ with $n \geq 3$, then we present it as

$$
\mathcal{O}^{\star}=\left\langle\tilde{\eta}, \tilde{u} \in \mathcal{O}^{\star} \mid \operatorname{ord}(\tilde{\eta})=n, \operatorname{ord}(\tilde{u})=2, \tilde{\eta} \tilde{u}=\tilde{u} \tilde{\eta}^{-1}\right\rangle .
$$

It has a unique maximal cyclic subgroup of order $n$, namely $\langle\tilde{\eta}\rangle$, which is normal. Let $C$ be a maximal cyclic subgroup of $\mathcal{O}^{\star}$ distinct from $\langle\tilde{\eta}\rangle$. Then $|C|=2$ and $C=\left\langle\tilde{u} \tilde{\eta}^{r}\right\rangle$ for some $0 \leq r<n$. If $n$ is odd, such subgroups form a single conjugacy class, and $N_{\mathcal{O}^{*}}(C)=C$; if $n$ is even, then they form two conjugacy classes according to the parity of $r$ (e.g. we may set $C^{\prime}=\langle\tilde{u}\rangle$ and $C^{\prime \prime}=\langle\tilde{u} \tilde{\eta}\rangle$ ), and $N_{\mathcal{O}^{\star}}(C) \neq C$ since $\tilde{\eta}^{n / 2}$ lies in the center of $\mathcal{O}^{\star}$. This proves part (3). The proof of the remaining parts are left to the interested reader. 
For the rest of this section, we fix a maximal order $\mathbb{O} \subset H$.

3.5. Denote the $H^{\times}$-conjugacy class of a maximal order $\mathbb{O}^{\prime} \subset H$ by $\llbracket \mathbb{O}^{\prime} \rrbracket$. There is a surjective map of finite sets

$$
\Upsilon: \mathrm{Cl}(\mathbb{O}) \rightarrow \operatorname{Tp}(H), \quad[I] \mapsto \llbracket \mathcal{O}_{l}(I) \rrbracket .
$$

The fibers of $\Upsilon$ is studied in [38, Section 1.7], whose result we briefly recall below.

By [33, Theorem 22.10], the set of nonzero two-sided fractional ideals of $\mathbb{O}$ forms a commutative multiplicative group $\mathscr{I}(\mathbb{O})$, which is a free abelian group generated by the nonzero prime two-sided ideals of $\mathbb{O}$. Let $\mathscr{P}(\mathbb{O}) \subseteq \mathscr{I}(\mathbb{O})$ be the subgroup of nonzero principal two-sided fractional ideals of $\mathbb{O}$, and $\mathscr{P}\left(O_{F}\right)$ the group of nonzero principal fractional $O_{F}$-ideals, identified with a subgroup of $\mathscr{P}(\mathbb{O})$ via $x O_{F} \mapsto x \mathbb{O}, \forall x \in F^{\times}$. For any maximal order $\mathbb{O}^{\prime}$, there is a bijection (see 38, Section 1.7], 40, Lemma III.5.6])

$$
\Upsilon^{-1}\left(\llbracket \mathbb{O}^{\prime} \rrbracket\right) \longleftrightarrow \mathscr{I}\left(\mathbb{O}^{\prime}\right) / \mathscr{P}\left(\mathbb{O}^{\prime}\right)
$$

The quotient group $\mathscr{I}\left(\mathbb{O}^{\prime}\right) / \mathscr{P}\left(\mathbb{O}^{\prime}\right)$ sits in a short exact sequence [13, Theorem 55.22]

$$
1 \rightarrow \mathcal{N}\left(\mathbb{O}^{\prime}\right) /\left(F^{\times} \mathbb{O}^{\prime \times}\right) \rightarrow \operatorname{Pic}\left(\mathbb{O}^{\prime}\right) \rightarrow \mathscr{I}\left(\mathbb{O}^{\prime}\right) / \mathscr{P}\left(\mathbb{O}^{\prime}\right) \rightarrow 1 .
$$

Here Pic $\left(\mathbb{O}^{\prime}\right)$ denotes the Picard group $\mathscr{I}\left(\mathbb{O}^{\prime}\right) / \mathscr{P}\left(O_{F}\right)$, whose cardinality can be calculated using the short exact sequence [13, Theorem 55.27]

$$
1 \rightarrow \mathrm{Cl}\left(O_{F}\right) \rightarrow \operatorname{Pic}\left(\mathbb{O}^{\prime}\right) \rightarrow \prod_{\mathfrak{p} \mid \mathfrak{d}(H)}(\mathbb{Z} / 2 \mathbb{Z}) \rightarrow 0,
$$

where $\mathrm{Cl}\left(O_{F}\right)$ denotes the ideal class group of $O_{F}$. It follows that

$$
\left|\Upsilon^{-1}\left(\llbracket \mathbb{O}^{\prime} \rrbracket\right)\right|=\frac{2^{\omega(H)} h(F)}{\left|\mathcal{N}\left(\mathbb{O}^{\prime}\right) /\left(F^{\times} \mathbb{O}^{\prime \times}\right)\right|},
$$

where $\omega(H)$ is the number of finite primes of $F$ that are ramified in $H$.

Remark 3.6. There is a natural action of $\mathrm{Cl}\left(O_{F}\right)$ on $\mathrm{Cl}(\mathbb{O})$ as follows:

$$
\mathrm{Cl}\left(O_{F}\right) \times \mathrm{Cl}(\mathbb{O}) \rightarrow \mathrm{Cl}(\mathbb{O}), \quad([\mathfrak{a}],[I]) \mapsto[\mathfrak{a} I] .
$$

Let $\overline{\mathrm{Cl}}(\mathbb{O}):=\mathrm{Cl}\left(O_{F}\right) \backslash \mathrm{Cl}(\mathbb{O})$ be the set of orbits of this action. Clearly, $\Upsilon([I])=$ $\Upsilon([\mathfrak{a} I])$, so $\Upsilon$ factors through $\overline{\mathrm{Cl}}(\mathbb{O})$. It is shown in [48, Section 3] that

- if $H$ splits at all finite places of $F$, then the induced map $\overline{\mathrm{Cl}}(\mathbb{O}) \rightarrow \operatorname{Tp}(H)$ is bijective;

- if $h(F)$ is odd, then the action in (3.14) is free.

This is the gist of the proof of Proposition 1.1, and we refer to [48, Section 3] for details.

3.7. We explain the strategy for calculating $h(G)=h(H, G)$ when $G=C_{n}$ is a cyclic group of order $n>1$. Let $\mathscr{B}_{n} \subseteq \mathscr{B}$ be the finite subset of CM $O_{F}$-orders $B$ such that $B^{\star} \simeq C_{n}$. For each fixed $B \in \mathscr{B}_{n}$, we define

$$
h\left(C_{n}, B\right):=\#\left\{[I] \in \mathrm{Cl}(\mathbb{O}) \mid \mathcal{O}_{l}(I)^{\star} \simeq C_{n} \text {, and } \operatorname{Emb}\left(B, \mathcal{O}_{l}(I)\right) \neq \emptyset\right\} .
$$

According to part (2) of Proposition 3.4.

$$
h\left(C_{n}\right)=\sum_{B \in \mathscr{B}_{n}} h\left(C_{n}, B\right) .
$$


We divide the type set $\operatorname{Tp}(H)$ into two subsets:

$\operatorname{Tp}^{\circ}(H):=\left\{\llbracket \mathbb{O}^{\prime} \rrbracket \in \operatorname{Tp}(H) \mid \mathbb{O}^{\prime \star}\right.$ is cyclic $\}, \quad$ and $\quad \operatorname{Tp}^{\natural}(H):=\operatorname{Tp}(H) \backslash \operatorname{Tp}^{\circ}(H)$.

In the trace formula (3.4), the summation $\sum_{[I] \in \mathrm{Cl}(\mathcal{O})} m\left(B, \mathcal{O}_{l}(I), \mathcal{O}_{l}(I)^{\times}\right)$is decomposed as two parts accordingly: one sums over all $[I] \in \mathrm{Cl}(\mathcal{O})$ with $\llbracket \mathcal{O}_{l}(I) \rrbracket \in$ $\operatorname{Tp}^{\circ}(H)$, and the other with $\llbracket \mathcal{O}_{l}(I) \rrbracket \in \mathrm{Tp}^{\natural}(H)$. Thanks to (3.7),

$$
\sum_{\substack{[I] \in \operatorname{Cl}(\mathbb{O}), \llbracket \mathcal{O}_{l}(I) \rrbracket \in \operatorname{Tp}^{\circ}(H)}} m\left(B, \mathcal{O}_{l}(I), \mathcal{O}_{l}(I)^{\times}\right)=2 h\left(C_{n}, B\right) .
$$

On the other hand, note that if $\Upsilon([I])=\Upsilon([J])$, then $\mathcal{O}_{l}(I) \simeq \mathcal{O}_{l}(J)$, so

$$
m\left(B, \mathcal{O}_{l}(I), \mathcal{O}_{l}(I)^{\times}\right)=m\left(B, \mathcal{O}_{l}(J), \mathcal{O}_{l}(J)^{\times}\right) .
$$

It follows that

$$
\sum_{\substack{[I] \in \operatorname{Cl}(\mathbb{O}), \llbracket \mathcal{O}_{l}(I) \rrbracket \in \operatorname{Tp}^{\natural}(H)}} m\left(B, \mathcal{O}_{l}(I), \mathcal{O}_{l}(I)^{\times}\right)=\sum_{\llbracket \mathbb{O}^{\prime} \rrbracket \in \operatorname{Tp}^{\natural}(H)}\left|\Upsilon^{-1}\left(\llbracket \mathbb{O}^{\prime} \rrbracket\right)\right| m\left(B, \mathbb{O}^{\prime}, \mathbb{O}^{\prime \times}\right) .
$$

Combining (3.4), (3.5), (3.13), (3.17) and (3.18), we obtain the following equation for each fixed $B \in \mathscr{B}_{n}$ :

$$
2 h\left(C_{n}, B\right)+2^{\omega(H)} h(F) \sum_{\llbracket \mathbb{O}^{\prime} \rrbracket \in \mathrm{T}^{\natural}(H)} \frac{m\left(B, \mathbb{O}^{\prime}, \mathbb{O}^{\prime \times}\right)}{\left|\mathcal{N}\left(\mathbb{O}^{\prime}\right) /\left(F^{\times} \mathbb{O}^{\prime \times}\right)\right|}=h(B) \prod_{\mathfrak{p} \mid \mathfrak{d}(H)}\left(1-\left(\frac{B}{\mathfrak{p}}\right)\right)
$$

Therefore, to compute $h\left(C_{n}, B\right)$ (and hence $h\left(C_{n}\right)$ ), it is crucial to classify all isomorphism classes of maximal orders with noncyclic reduced unit groups, and ideally, to write them down as explicitly as possible. Sections 46 are devoted to this task. Once this is done, then for any noncyclic group $G$,

$$
h(G)=\sum \frac{2^{\omega(H)} h(F)}{\left|\mathcal{N}\left(\mathbb{O}^{\prime}\right) /\left(F^{\times} \mathbb{O}^{\prime \times}\right)\right|},
$$

where the summation runs over all $\llbracket \mathbb{O}^{\prime} \rrbracket \in \mathrm{Tp}^{\natural}(H)$ with $\mathbb{O}^{\prime \star} \simeq G$.

3.8. Lastly, we compute $h\left(C_{1}\right)$ by the mass formula. Recall that the mass of an arbitrary $O_{F}$-order $\mathcal{O} \subset H$ is defined as

$$
\operatorname{Mass}(\mathcal{O}):=\sum_{[I] \in \mathrm{Cl}(\mathcal{O})} \frac{1}{\left|\mathcal{O}_{l}(I)^{\star}\right|} .
$$

The mass of a maximal order $\mathbb{O}$ can be calculated by the mass formula 40, Corollaire V.2.3]

$$
\operatorname{Mass}(\mathbb{O})=\frac{h(F)\left|\zeta_{F}(-1)\right|}{2^{([F: \mathbb{Q}]-1)}} \prod_{\mathfrak{p} \mid \mathfrak{d}(H)}(N(\mathfrak{p})-1) .
$$

See [44, Lemma 5.1.2] for the mass formula of an arbitrary $O_{F}$-order in $H$. Once $h(G)$ is known for every nontrivial finite group $G$, then we have

$$
h\left(C_{1}\right)=\operatorname{Mass}(\mathbb{O})-\sum_{G \neq C_{1}} \frac{h(G)}{|G|} .
$$


Alternatively, one could compute $h\left(C_{1}\right)$ by

$$
h\left(C_{1}\right)=h(\mathbb{O})-\sum_{G \neq C_{1}} h(G) .
$$

According to Eichler's class number formula [40, Corollaire V.2.5],

$$
h(\mathcal{O})=\operatorname{Mass}(\mathcal{O})+\frac{1}{2} \sum_{B \in \mathscr{B}} h(B)\left(1-w(B)^{-1}\right) \prod_{\mathfrak{p}} m_{\mathfrak{p}}(B),
$$

where $w(B)=\left[B^{\times}: O_{F}^{\times}\right]$as in (3.1), and $m_{\mathfrak{p}}(B)$ is the number of conjugacy classes of local optimal embeddings at $\mathfrak{p}$ as in (3.3). A similar formula [44, Theorem 1.5] holds for arbitrary $\mathbb{Z}$-orders of full rank in $H$. When $\mathcal{O}=\mathbb{O}$ is maximal, we have

$$
h(\mathbb{O})=\frac{h(F)\left|\zeta_{F}(-1)\right|}{2^{([F: \mathbb{Q}]-1)}} \prod_{\mathfrak{p} \mid \mathfrak{d}(H)}(N(\mathfrak{p})-1)+\frac{1}{2} \sum_{B \in \mathscr{B}} h(B)\left(1-w(B)^{-1}\right) \prod_{\mathfrak{p} \mid \mathfrak{d}(H)}\left(1-\left(\frac{B}{\mathfrak{p}}\right)\right) .
$$

Applying (3.25) for real quadratic fields $F$, Vignéras obtains explicit class number formulas for all Eichler orders of square free level in [38, Theorem 3.1].

Comparing (3.26) with (3.19), we see that (3.23) is more direct than (3.24) since it avoid calculating the right hand side of (3.19) twice. However, the two approaches share the same theoretical root (3.4), so the difference is merely superficial.

\section{Minimal $G$-ORDers}

Throughout this section, $F$ is a totally real field, and $H$ is a totally definite quaternion $F$-algebra. We mainly focus on the case that $F=\mathbb{Q}(\sqrt{d})$ is a real quadratic field with square-free $d \geq 6$. The cases $d \in\{2,3,5\}$ are treated separately in Section 8.1. The fundamental unit of $F=\mathbb{Q}(\sqrt{d})$ is denoted by $\varepsilon$. By definition, $\varepsilon>1$ for the canonical embedding $F \hookrightarrow \mathbb{R}$.

4.1. Vignéras unit index of $\mathcal{O}$ and the fundamental unit of $F=\mathbb{Q}(\sqrt{d})$. For an $O_{F}$-order $\mathcal{O}$ in $H$, we set $\mathcal{O}^{1}:=\left\{u \in \mathcal{O}^{\times} \mid \operatorname{Nr}(u)=1\right\}$. It is known [40, Section V.1] that $\mathcal{O}^{1}$ is a finite normal subgroup of $\mathcal{O}^{\times}$. Vignéras shows in 39, Theorem 6] that $\left[\mathcal{O}^{\times}: O_{F}^{\times} \mathcal{O}^{1}\right] \in\{1,2,4\}$ for any arbitrary totally real field $F$, so we call it the Vignéras unit index of $\mathcal{O}$.

Lemma 4.1.1. If $F=\mathbb{Q}(\sqrt{d})$ is a real quadratic field, then $\left[\mathcal{O}^{\times}: O_{F}^{\times} \mathcal{O}^{1}\right] \in\{1,2\}$. Moreover, if $\mathrm{N}_{F / \mathbb{Q}}(\varepsilon)=-1$, then $\mathcal{O}^{\times}=O_{F}^{\times} \mathcal{O}^{1}$.

Proof. Since $H$ is totally definite, we have $O_{F}^{\times 2} \subseteq \operatorname{Nr}\left(\mathcal{O}^{\times}\right) \subseteq O_{F,+}^{\times}$, the group of totally positive units in $O_{F}^{\times}$. This gives rise to an embedding

$$
\mathcal{O}^{\times} /\left(O_{F}^{\times} \mathcal{O}^{1}\right) \hookrightarrow O_{F,+}^{\times} / O_{F}^{\times 2} .
$$

When $F$ is a real quadratic field, the fundamental unit $\varepsilon \in O_{F}^{\times}$is totally positive if and only if $\mathrm{N}_{F / \mathbb{Q}}(\varepsilon)=1$. We have

$$
O_{F,+}^{\times}=\left\{\begin{array}{ll}
\langle\varepsilon\rangle & \text { if } \mathrm{N}_{F / \mathbb{Q}}(\varepsilon)=1, \\
\left\langle\varepsilon^{2}\right\rangle & \text { if } \mathrm{N}_{F / \mathbb{Q}}(\varepsilon)=-1,
\end{array} \quad \text { while } \quad O_{F}^{\times 2}=\left\langle\varepsilon^{2}\right\rangle .\right.
$$

The lemma follows directly from (4.1).

We set $\mathscr{S}=\{1, \varepsilon\}$ if $\mathrm{N}_{F / \mathbb{Q}}(\varepsilon)=1$, and $\mathscr{S}=\{1\}$ otherwise. Thus $\mathscr{S}$ is a complete set of representatives of $O_{F,+}^{\times} / O_{F}^{\times 2}$ for $F=\mathbb{Q}(\sqrt{d})$. 
Remark 4.1.2. It is well-known [1, Section 11.5] that for $F=\mathbb{Q}(\sqrt{d})$,

- $\mathrm{N}_{F / \mathbb{Q}}(\varepsilon)=1$ if $d$ is divisible by a prime $p \equiv 3(\bmod 4)$;

- $\mathrm{N}_{F / \mathbb{Q}}(\varepsilon)=-1$ if $d=p$ with a prime $p \equiv 1(\bmod 4)$, or $d=2 p$ with a prime $p \equiv 5(\bmod 8)$, or $d=p_{1} p_{2}$ with primes $p_{1}, p_{2} \equiv 1(\bmod 4)$ and $\left(\frac{p_{1}}{p_{2}}\right)=\left(\frac{p_{2}}{p_{1}}\right)=-1$.

Before the discussion leads us too far astray, we refer to the classes of $d$ with known signs of $\mathrm{N}_{F / \mathbb{Q}}(\varepsilon)$ in Corollaries 21.10 and 24.5 of [11].

4.2. Structure of the reduced unit group of a CM-extension $K / F$. We first return to the general case where $F$ is an arbitrary totally real number field. Let $K / F$ be a CM-extension, and $\boldsymbol{\mu}(K)$ be the group of roots of unity in $K$. By [41, Theorem 4.12], the Hasse unit index

$$
Q_{K / F}:=\left[O_{K}^{\times}: O_{F}^{\times} \boldsymbol{\mu}(K)\right]
$$

is either 1 or 2. Following [11, Section 13], we call $K / F$ a $C M$-extension of type $I$ if $Q_{K / F}=1$, and of type II if $Q_{K / F}=2$. Recall that an $O_{F}$-order in a CM-extension of $F$ is called a $C M O_{F}$-order.

Lemma 4.2.1. The reduced unit group $O_{K}^{\star}:=O_{K}^{\times} / O_{F}^{\times}$is a cyclic group of order $Q_{K / F}|\boldsymbol{\mu}(K)| / 2$. In particular, $B^{\star}:=B^{\times} / O_{F}^{\times}$is a cyclic subgroup of $O_{K}^{\star}$ for every $C M O_{F}$-order $B$.

Proof. Note that $\boldsymbol{\mu}(K) /\{ \pm 1\} \subseteq O_{K}^{\star}$ is a cyclic subgroup of index $Q_{K / F} \in\{1,2\}$. The lemma is true if one of the following conditions holds:

- $K / F$ is of type $\mathrm{I}$;

- $K \neq F(\sqrt{-1})$ so that $\boldsymbol{\mu}(K) /\{ \pm 1\}$ has odd order.

Now suppose that $K=F(\sqrt{-1})$ and $Q_{K / F}=2$. If $u \in O_{K}^{\times}$represents an element of order 2 in $O_{K}^{\star}$, then it is purely imaginary, i.e. $u \sqrt{-1} \in O_{F}^{\times}$. Thus $O_{K}^{\star}$ contains a unique element of order 2 represented by $\sqrt{-1}$, so it must be cyclic.

Since $\left[O_{K}^{\star}: \boldsymbol{\mu}(K) /\{ \pm 1\}\right] \in\{1,2\}$, if $\tilde{u} \in O_{K}^{\star}$ has odd order, then $\tilde{u} \in \boldsymbol{\mu}(K) /\{ \pm 1\}$ and is represented by a root of unity.

From now on, we assume that $F=\mathbb{Q}(\sqrt{d})$ is a quadratic real field, where $d \in \mathbb{N}$ is a positive square free integer. Since $[K: \mathbb{Q}]=4$, the possible orders of $\boldsymbol{\mu}(K)$ are $2,4,6,8,10,12$ by [45, Section 2.3]. Moreover,

- $|\boldsymbol{\mu}(K)|=8$ if and only if $F=\mathbb{Q}(\sqrt{2})$ and $K=\mathbb{Q}(\sqrt{2}, \sqrt{-1})$;

- $|\boldsymbol{\mu}(K)|=10$ if and only if $F=\mathbb{Q}(\sqrt{5})$ and $K=\mathbb{Q}\left(\zeta_{10}\right)$;

- $|\boldsymbol{\mu}(K)|=12$ if and only if $F=\mathbb{Q}(\sqrt{3})$ and $K=\mathbb{Q}(\sqrt{3}, \sqrt{-1})$.

Here $\zeta_{n}$ denotes the primitive $n$-th root of unity $e^{2 \pi i / n}$ for all positive $n \in \mathbb{N}$.

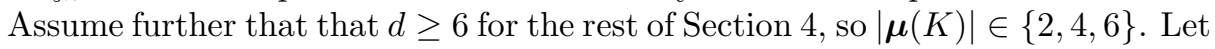
$K / F$ be a CM-extension with $\left[O_{K}^{\times}: O_{F}^{\times}\right]>1$. If $\boldsymbol{\mu}(K)=\{ \pm 1\}$, then $\mathrm{N}_{F / \mathbb{Q}}(\varepsilon)=1$ and $K=F(\sqrt{-\varepsilon})$ by [45, Lemma 2.2]. It follows that $\left[O_{K}^{\times}: O_{F}^{\times}\right]>1$ if and only if

$$
K= \begin{cases}F(\sqrt{-1}) \text { or } F(\sqrt{-3}) & \text { if } \mathrm{N}_{F / \mathbb{Q}}(\varepsilon)=-1 \\ F(\sqrt{-1}), F(\sqrt{-\varepsilon}), \text { or } F(\sqrt{-3}) & \text { if } \mathrm{N}_{F / \mathbb{Q}}(\varepsilon)=1\end{cases}
$$

Clearly, $F(\sqrt{-\varepsilon}) \neq F(\sqrt{-1})$ for all $F$. On the other hand, $F(\sqrt{-\varepsilon})=F(\sqrt{-3})$ if $3 \varepsilon \in F^{\times 2}$.

By Lemma 4.2.1 $O_{K}^{\star}:=O_{K}^{\times} / O_{F}^{\times}$is a cyclic group of order $n \in\{2,3,4,6\}$ and

$$
n=4(\text { resp. } 6) \Leftrightarrow K=F(\sqrt{-1})(\text { resp. } F(\sqrt{-3})) \text { and } Q_{K / F}=2 \text {. }
$$


According to [9, Lemma 2], $F(\sqrt{-1}) / F$ (resp. $F(\sqrt{-3}) / F$ ) is of type II if and only if $2 \varepsilon \in F^{\times 2}$ (resp. $3 \varepsilon \in F^{\times 2}$ ). For example, we have $2 \varepsilon \in F^{\times 2}$ if $d=c p$ with $c \in\{1,2\}$ and $p \equiv 3(\bmod 4)$ by [27, Lemma 3] or [50, Lemma 3.2(1)]. Similarly, $3 \varepsilon \in F^{\times 2}$ if $d=3 p$ with $p \equiv 3(\bmod 4)$ by $[9$, Lemma 3$]$. We introduce the following notation:

- if $2 \varepsilon \in F^{\times 2}$, then we fix $\vartheta \in F^{\times}$such that $\varepsilon=2 \vartheta^{2}$;

- if $3 \varepsilon \in F^{\times 2}$, then we fix $\varsigma \in F^{\times}$such that $\varepsilon=3 \varsigma^{2}$.

Note that in these two cases, 2 (resp. 3 ) is ramified in $F / \mathbb{Q}$, and $2 \vartheta$ (resp. $3 \varsigma$ ) generates the unique prime of $F$ over 2 (resp. 3 ). Note that $2 \varepsilon$ and $3 \varepsilon$ are simultaneously perfect squares in $F^{\times}$if and only if $d=6$. Lastly, if $\{2 \varepsilon, 3 \varepsilon\} \cap F^{\times 2}=\emptyset$, then $\left|O_{K}^{\star}\right| \in\{2,3\}$. For instance, if $\mathrm{N}_{F / \mathbb{Q}}(\varepsilon)=-1$, then $\{2 \varepsilon, 3 \varepsilon\} \cap F^{\times 2}=\emptyset$, and every CM-extension $K / F$ is of type I by [11, Lemma 13.6].

We select a special set of representatives of $O_{K}^{\star}$. The representative of the identity element is always chosen to be 1 . Let $\tilde{u} \in O_{K}^{\star}$ be a nontrivial element. After multiplying a representative $u \in O_{K}^{\times}$by a suitable element of $O_{F}^{\times}$, we may assume that $n_{u}:=\mathrm{N}_{K / F}(u)$ belongs to the set $\mathscr{S} \subseteq\{1, \varepsilon\}$. This determines $u$ uniquely up to sign and we put $\mathrm{N}_{K / F}(\tilde{u}):=n_{u}$. Let $P_{u}(x)=x^{2}-t_{u} x+n_{u} \in F[x]$ be the minimal polynomial of $u$ over $F$. Changing $u$ to $-u$ switches the sign of $t_{u}$ and leaves $n_{u}$ invariant, so we put $P_{\tilde{u}}(x)=x^{2} \pm t_{u} x+n_{u}$. In Table 4.1 we list all the possible $P_{\tilde{u}}(x)$ for each $\tilde{u}$. The method is the same as that of [45, Lemma 2.2], hence omitted.

TABLE 4.1. Minimal polynomials of $\tilde{u}$ when $d \geq 6$

\begin{tabular}{|c|c|c|c|}
\hline $\operatorname{ord}(\tilde{u})$ & Conditions & $P_{\tilde{u}}(x) \in F[x]$ & roots of $P_{\tilde{u}}(x)$ \\
\hline \multirow{2}{*}{2} & $\mathrm{~N}_{K / F}(\tilde{u})=1$ & $x^{2}+1$ & $\pm \sqrt{-1}$ \\
\cline { 2 - 4 } & $\mathrm{N}_{F / \mathbb{Q}}(\varepsilon)=1, \mathrm{~N}_{K / F}(\tilde{u})=\varepsilon$ & $x^{2}+\varepsilon$ & $\pm \sqrt{-\varepsilon}$ \\
\hline 3 & & $x^{2} \pm x+1$ & $\pm \zeta_{3}^{ \pm 1}$ \\
\hline 4 & $2 \varepsilon \in F^{\times 2}$ & $x^{2} \pm 2 \vartheta x+\varepsilon$ & $\pm \sqrt{ \pm \varepsilon \sqrt{-1}}= \pm \vartheta(1 \pm \sqrt{-1})$ \\
\hline 6 & $3 \varepsilon \in F^{\times 2}$ & $x^{2} \pm 3 \varsigma x+\varepsilon$ & $\pm \sqrt{\varepsilon \zeta_{6}^{ \pm 1}}= \pm \varsigma \zeta_{6}^{ \pm 1} \sqrt{-3}$ \\
\hline
\end{tabular}

4.3. Finite noncyclic subgroups of $H^{\times} / O_{F}^{\times}$. We keep the assumption that $F=$ $\mathbb{Q}(\sqrt{d})$ with $d \geq 6$. For every $O_{F}$-order $\mathcal{O}$ in $H$, the reduced unit group $\mathcal{O}^{\star}=$ $\mathcal{O}^{\times} / O_{F}^{\times}$is a finite subgroup of $H^{\times} / O_{F}^{\times}$, so we study the finite subgroups of $H^{\times} / O_{F}^{\times}$.

Let $\tilde{u} \in H^{\times} / O_{F}^{\times}$be a nontrivial element. For any $u \in H^{\times}$representing $\tilde{u}$, the subfield $F(u) \subset H$ depends only on $\tilde{u}$ and is denoted by $F(\tilde{u})$. Since $H$ is totally definite, $F(\tilde{u}) / F$ is a CM-extension. Note that $\tilde{u}$ has finite order if and only if $u$ is a unit of $O_{F(\tilde{u})}$.

Definition 4.3.1. Let $G$ be a finite noncyclic subgroup of $H^{\times} / O_{F}^{\times}$. The $O_{F^{-}}$ submodule $\mathcal{O}$ spanned by a complete set of representatives of $G$ is an $O_{F}$-order in $H$ and called the minimal $O_{F}$-order attached to $G$, or minimal $G$-order for short. 
Clearly, the minimal $G$-order $\mathcal{O}$ does not depend on the choice of representatives, and $G$ is naturally a subgroup of $\mathcal{O}^{\star}$. We will show that in fact $G=\mathcal{O}^{\star}$ in Corollary 4.4.4. For the moment, let us note that $\operatorname{ord}(\tilde{u}) \in\{2,3,4,6\}$ for every nontrivial $\tilde{u} \in G$ by Section 4.2. Applying the classification in Section 3.1, we write down all possible finite noncyclic subgroups of $H^{\times} / O_{F}^{\times}$up to isomorphism in Table 4.2

TABLE 4.2. Finite noncyclic subgroups of $H^{\times} / O_{F}^{\times}$

\begin{tabular}{|c|c|c|}
\hline$F=\mathbb{Q}(\sqrt{d}), d \geq 6$ & $\operatorname{ord}(\tilde{u})>1$ & possible noncyclic $G$ \\
\hline $2 \varepsilon \in F^{\times 2}$ & $2,3,4$ & $D_{2}, D_{3}, D_{4}, A_{4}, S_{4}$ \\
\hline $3 \varepsilon \in F^{\times 2}$ & $2,3,6$ & $D_{2}, D_{3}, D_{6}, A_{4}$ \\
\hline$\{2 \varepsilon, 3 \varepsilon\} \cap F^{\times 2}=\emptyset$ & 2,3 & $D_{2}, D_{3}, A_{4}$ \\
\hline
\end{tabular}

Definition 4.3.2. For $s \in\{1,2\}$, let $H_{s}$ be a totally definite quaternion $F$-algebra, and $G_{s}$ be a finite noncyclic subgroup of $H_{s}^{\times} / O_{F}^{\times}$. We say $G_{1}$ and $G_{2}$ are strictly isomorphic if there is an $F$-isomorphism of quaternion algebras

$$
\psi: H_{1} \rightarrow H_{2} \text { such that } \widetilde{\psi}\left(G_{1}\right)=G_{2},
$$

where $\widetilde{\psi}: H_{1}^{\times} / O_{F}^{\times} \rightarrow H_{2}^{\times} / O_{F}^{\times}$denotes the induced isomorphism.

By transport of structure, strictly isomorphic groups have isomorphic minimal $O_{F}$-orders. We characterize strict isomorphisms using the reduced norm map.

For every finite subgroup $G$ in $H^{\times} / O_{F}^{\times}$, the reduced norm map induces a homomorphism

$$
\mathrm{Nr}: G \rightarrow O_{F,+}^{\times} / O_{F}^{\times 2} \text {. }
$$

Recall that $O_{F,+}^{\times} / O_{F}^{\times 2}$ is represented by $\mathscr{S}$, which is either $\{1, \varepsilon\}$ or $\{1\}$ depending on whether $\mathrm{N}_{F / \mathbb{Q}}(\varepsilon)=1$ or not. Given $\tilde{u} \in G$, we may always pick a representative $u \in O_{F(\tilde{u})}^{\times}$of $\tilde{u}$ such that $\operatorname{Nr}(u)=\mathrm{N}_{F(\tilde{u}) / F}(u) \in \mathscr{S} \subseteq\{1, \varepsilon\}$. Subsequently, representatives of $\tilde{u}$ refer exclusively to these ones. We set $\operatorname{Nr}(\tilde{u}):=\operatorname{Nr}(u)$ and call it the reduced norm of $\tilde{u}$. If $\widetilde{\psi}: G_{1} \rightarrow G_{2}$ is a strict isomorphism as in (4.5), then clearly $\mathrm{Nr} \circ \widetilde{\psi}=\mathrm{Nr}$.

Definition 4.3.3. Let $G$ be a finite noncyclic subgroup of $H^{\times} / O_{F}^{\times}$isomorphic to $D_{n}$ with $n \in\{2,3\}$. We say $G$ is of the first kind and write $G \simeq D_{n}^{\dagger}$ if every element of order 2 in $G$ has reduced norm 1; otherwise, we say $G$ is of the second kind and write $G \simeq D_{n}^{\ddagger}$.

Remark 4.3.4. We explain why Definition 4.3 .3 applies only to $D_{2}$ and $D_{3}$ but not to any other groups in Table 4.2. Clearly, the reduced norm map in (4.6) is constant on each conjugacy class of $G$. If $G$ contains an element of order 4 or 6 , then $\mathscr{S}=\{1, \varepsilon\}$ and $\mathrm{Nr}$ is surjective by Table 4.1. We separate the rest of the discussion into cases:

(1) If $G \simeq A_{4}$, then $\operatorname{Nr}(G)=\{1\}$ since $A_{4}$ contains no subgroup of index 2 .

(2) Suppose that $G \simeq S_{4}$. Any two isomorphisms $G \simeq S_{4}$ differ by a conjugation because all automorphisms of $S_{4}$ are inner. In particular, it makes sense to talk about cycle types [43, Section 2.3.1] of elements of $G$. There are two conjugacy classes of elements of order 2 in $S_{4}$ : 
- the transpositions, and

- the permutations of type $(2,2)$.

The group $G$ has a unique subgroup $G^{\prime}$ of index 2 which corresponds to $A_{4}$ for every isomorphism $G \simeq S_{4}$. We have $\operatorname{ker}(\mathrm{Nr})=G^{\prime}$ since $\mathrm{Nr}$ maps surjectively onto $O_{F,+}^{\times} / O_{F}^{\times 2} \simeq \mathbb{Z} / 2 \mathbb{Z}$ and $\operatorname{ker}(\mathrm{Nr}) \supseteq G^{\prime}$. It follows that every transposition has reduced norm $\varepsilon$, and every permutation of type $(2,2)$ has reduced norm 1 .

(3) Suppose that $G \simeq D_{n}$ with $n \in\{2,3,4,6\}$. We present $G$ as in (3.8):

$$
G=\left\langle\tilde{\eta}, \tilde{u} \in G \mid \operatorname{ord}(\tilde{\eta})=n, \operatorname{ord}(\tilde{u})=2, \tilde{\eta} \tilde{u}=\tilde{u} \tilde{\eta}^{-1}\right\rangle .
$$

First consider the case $n=2 m$ with $m \in\{2,3\}$. There are 3 conjugacy classes of elements of order 2 in $G$ :

- $\left\{\tilde{\eta}^{m}\right\}$, consisting of the unique nontrivial element of the center;

- $\left\{\tilde{u} \tilde{\eta}^{2 t} \mid 0 \leq t \leq m-1\right\}$

- $\left\{\tilde{u} \tilde{\eta}^{2 t+1} \mid 0 \leq t \leq m-1\right\}$.

We have $\operatorname{Nr}(\tilde{u}) \neq \operatorname{Nr}(\tilde{u} \tilde{\eta})$ since $\operatorname{Nr}(\tilde{\eta})=\varepsilon$. Replacing $\tilde{u}$ by $\tilde{u} \tilde{\eta}$ if necessary, we may and will assume that

$$
\operatorname{Nr}(\tilde{u})=1 \quad \text { and } \quad \operatorname{Nr}(\tilde{u} \tilde{\eta})=\varepsilon .
$$

If $G \simeq D_{3}$, then there is a unique conjugacy class of elements of 2 , which has a uniform reduced norm (either 1 or $\varepsilon$ ). These two cases are distinguished by the notation $D_{3}^{\dagger}$ and $D_{3}^{\ddagger}$.

Lastly, suppose that $G \simeq D_{2}$, the Klein 4-group. We write $G \simeq D_{2}^{\dagger}$ if every element of $G$ has reduced norm 1 . If $G \not D_{2}^{\dagger}$, then there is an element with reduced norm $\varepsilon$, which we choose as $\tilde{\eta}$. Once again $\operatorname{Nr}(\tilde{u}) \neq \operatorname{Nr}(\tilde{u} \tilde{\eta})$. Replacing $\tilde{u}$ by $\tilde{u} \tilde{\eta}$ if necessary, we always assume that (4.8) holds true in this case as well.

Proposition 4.3.5. For $s \in\{1,2\}$, let $H_{s}$ and $G_{s}$ be as in Definition 4.3.2. Then $G_{1}$ and $G_{2}$ are strictly isomorphic if and only if they are isomorphic as abstract groups and are of the same kind (if applicable).

Proof. We first prove the proposition under the assumption that $G_{s} \simeq D_{n}$ with $n \in\{2,3,4,6\}$. Presented $G_{s}$ as in (4.7), and assume the generators $\left\{\tilde{\eta}_{s}, \tilde{u}_{s}\right\}_{s=1,2}$ satisfy (4.8) if $G_{s} \simeq D_{2}^{\ddagger}$ or $G_{s} \simeq D_{n}$ with $n \in\{4,6\}$. By the assumption, we have $\operatorname{ord}\left(\tilde{\eta}_{1}\right)=\operatorname{ord}\left(\tilde{\eta}_{2}\right)$ and $\operatorname{Nr}\left(\tilde{\eta}_{1}\right)=\operatorname{Nr}\left(\tilde{\eta}_{2}\right)$. Let $K_{s}:=F\left(\tilde{\eta}_{s}\right)$ for $s=1$, 2. Thanks to Table 4.1, there is an $F$-isomorphism of CM-fields

$$
\psi_{0}: K_{1} \rightarrow K_{2} \text { such that } \widetilde{\psi}_{0}\left(\tilde{\eta}_{1}\right)=\tilde{\eta}_{2} .
$$

Let $u_{s} \in H_{s}^{\times}$be a representative of $\tilde{u}_{s}$ and put $c:=u_{1}^{2}=u_{2}^{2} \in\{-1,-\varepsilon\}$. We have $u_{s} K_{s} u_{s}^{-1}=K_{s}$ since $\tilde{u}_{s} \tilde{\eta}_{s} \tilde{u}_{s}^{-1}=\tilde{\eta}_{s}^{-1}$. Hence conjugation by $u_{s}$ induces the unique nontrivial $F$-automorphism $y_{s} \mapsto \bar{y}_{s}$ on $K_{s}$. It follows that $H_{s}=K_{s}+$ $K_{s} u_{s} \simeq\left\{K_{s}, c\right\}$ (notation as in the start of Section 2). Thus $\psi_{0}$ extends to an $F$-isomorphism

$$
\psi: H_{1} \rightarrow H_{2} \quad \text { such that } \quad \psi\left(u_{1}\right)=u_{2} .
$$

Next, assume that $G_{1}$ and $G_{2}$ are both isomorphic to $A_{4}$. By Remark 4.3.4, we have $A_{4} \supset D_{2}^{\dagger}$. Applying the preceding proof to $D_{2}^{\dagger}$, we may identify both $H_{s}$ with 
$H:=\{F(\sqrt{-1}),-1\}=\left(\frac{-1,-1}{F}\right)$ such that the unique Sylow 2-subgroup of $G_{s}$ is identified with $V:=\{\tilde{1}, \tilde{i}, \tilde{j}, \tilde{k}\} \subseteq H^{\times} / O_{F}^{\times}$. Put

$$
\xi:=(1+i+j+k) / 2 \in\left(\frac{-1,-1}{\mathbb{Q}}\right) \subset\left(\frac{-1,-1}{F}\right) .
$$

The subgroup $G_{0}:=\langle\tilde{i}, \tilde{j}, \tilde{\xi}\rangle \subset H^{\times} / O_{F}^{\times}$is isomorphic to $A_{4}$. Let $G \subset H^{\times} / O_{F}^{\times}$ be a subgroup such that $G \supset V$ and $G \simeq A_{4}$. We show that $G=G_{0}$. The inner automorphisms of $G$ induce an embedding

$$
G / V \hookrightarrow \operatorname{Aut}(V) \simeq S_{3},
$$

which identifies $G / V$ with $A_{3} \subset S_{3}$. In particular, there exists an element $\tilde{\xi}^{\prime} \in G$ of order 3 such that conjugation by $\tilde{\xi}^{\prime}$ induces the cyclic permutation $(\tilde{i}, \tilde{j}, \tilde{k})$. On the other hand, conjugation by $\tilde{\xi}$ also acts as $(\tilde{i}, \tilde{j}, \tilde{k})$. It follows that $\xi^{-1} \xi^{\prime}$ commutes with $i, j, k$ up to sign, and hence $\xi^{-1} \xi^{\prime} \in F^{\times}\langle i, j, k\rangle$, the subgroup of $H^{\times}$generated by $F^{\times}$and $\{i, j, k\}$. By Table 4.1 $\operatorname{Nr}\left(\xi^{\prime}\right)=1$ since $\operatorname{ord}\left(\tilde{\xi}^{\prime}\right)=3$. We have $\xi^{-1} \xi^{\prime} \in$ $\{ \pm 1, \pm i, \pm j, \pm k\}$ and hence $\tilde{\xi} \in G$. This concludes the proof for $A_{4}$.

Lastly, suppose that $G_{1}$ and $G_{2}$ are both isomorphic to $S_{4}$. By Table 4.2, we have $2 \varepsilon \in F^{\times 2}$. Since $S_{4} \supset A_{4}$, we may identify each $H_{s}$ with $H=\left(\frac{-1,-1}{F}\right)$ in such a way that the unique index 2 subgroup of $G_{s}$ is identified with $G_{0} \simeq A_{4}$ as above. It remains to show that there is a unique subgroup of $H^{\times} / O_{F}^{\times}$containing $G_{0}$ and isomorphic to $S_{4}$. Let $G$ be such a group. Then $\tilde{i} \in V \subset G$ is the square of an element of order 4 , say $\tilde{\tau} \in G$. We then have $F(\tilde{\tau})=F(i)$ and $\tilde{\tau}$ is represented by either $\vartheta(1+i)$ or $\vartheta(1-i)$ according to Table 4.1. These two elements are mutually inverse to each other modulo $O_{F}^{\times}$. Thus $G$ is generated by $G_{0}$ and $\vartheta(1+i) O_{F}^{\times}$, hence uniquely determined.

In the case $G \simeq S_{4}$ and $G \supset G_{0}$, the same argument also shows that $\vartheta(1+j) O_{F}^{\times} \in$ $G$ as well. This will be used when we write down the minimal $G$-order in Section 4.4 .

By Table 4.2 and Proposition 4.3.5 the strict isomorphism classes of all possible finite noncyclic subgroups of $H^{\times} / O_{F}^{\times}$for all $H$ are

$$
\mathcal{G}^{\natural}:=\left\{D_{2}^{\dagger}, D_{2}^{\ddagger}, D_{3}^{\dagger}, D_{3}^{\ddagger}, D_{4}, D_{6}, A_{4}, S_{4}\right\} .
$$

Given two distinct groups $G, G^{\prime} \in \mathcal{G}^{\natural}$, we write $G<G^{\prime}$ if $G$ is realizable as a subgroup of $G^{\prime}$. The relationships between these groups are illustrated in Figure 4.1 where every pair $G<G^{\prime}$ is connected by a line.

Figure 4.1. Relationships between the groups in $\mathcal{G}^{\natural}$

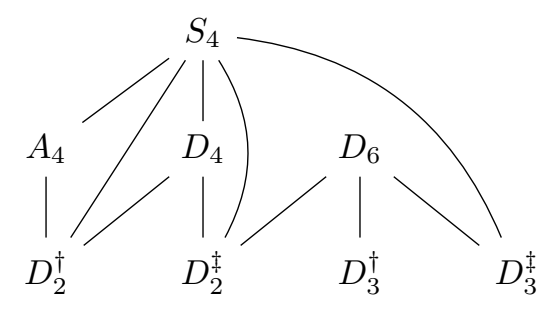

The diagram makes heavy use of Remark 4.3.4 For example, $D_{3}^{\dagger}$ is not realizable as a subgroup of $S_{4}$. Otherwise $D_{3}^{\dagger}<A_{4}$, but $A_{4}$ does not contain any 
subgroup of index 2. The group $D_{6}$ has a unique conjugacy class of subgroups of order 4, namely its Sylow 2-subgroups. Each Sylow 2-subgroup contains the unique nontrivial element of the center, which has reduced norm $\varepsilon$. Hence $D_{6}$ contains subgroups strictly isomorphic to $D_{2}^{\ddagger}$ but none to $D_{2}^{\dagger}$. Lastly, let $\{\tilde{u}, \tilde{\eta}\}$ be generators of $D_{4}$ as in (4.7) with $\operatorname{Nr}(\tilde{u})=1$. Since $\operatorname{Nr}\left(\tilde{\eta}^{2}\right)=1$, we have $\left\langle\tilde{u}, \tilde{\eta}^{2}\right\rangle \simeq D_{2}^{\dagger}$ and $\left\langle\tilde{u} \tilde{\eta}, \tilde{\eta}^{2}\right\rangle \simeq D_{2}^{\ddagger}$. We leave the discussion of the remaining cases to the interested readers.

Lemma 4.3.6. Let $G$ be a finite noncyclic subgroup of $H^{\times} / O_{F}^{\times}$, and $G_{1}, G_{2}$ be two noncyclic subgroups of $G$. If $G_{1}$ and $G_{2}$ are strictly isomorphic, then they are conjugate in $G$.

Proof. Suppose that $G_{1}$ and $G_{2}$ are strictly isomorphic. First, we have $G_{1}=G_{2}$ in the following cases:

- $G \simeq A_{4}, D_{4}$

- $G \simeq S_{4}$ and $G_{s} \simeq A_{4}$ (or $D_{2}^{\dagger}$ ) for $s=1,2$;

- $G \simeq D_{6}$ and $G_{s} \simeq D_{3}^{\dagger}$ (or $D_{3}^{\ddagger}$ ) for $s=1,2$.

Next, if $G \simeq S_{4}$ (resp. $\left.D_{6}\right)$ and $G_{s} \simeq D_{4}\left(\right.$ resp. $\left.D_{2}^{\ddagger}\right)$, then both $G_{s}$ are Sylow 2subgroups of $G$, and hence conjugate. It remains to handle the cases that $G \simeq S_{4}$, and $G_{s} \simeq D_{2}^{\ddagger}$ or $D_{3}^{\ddagger}$. Note that a noncyclic order 4 subgroup of $S_{4}$ is strictly isomorphic to $D_{2}^{\ddagger}$ if and only if it is not normal, and such subgroups form a single conjugacy class. There is a single conjugacy class of subgroups of order 6 in $S_{4}$, and each such subgroup is strictly isomorphic to $D_{3}^{\ddagger}$.

\subsection{Structure of the minimal $G$-orders for noncyclic finite groups $G$.}

Definition 4.4.1. For each $G \in \mathcal{G}^{\natural}$ as in (4.12), an $O_{F}$-order $\mathcal{O} \subset H$ is called a minimal $G$-order if there exists $G^{\prime} \subset H^{\times} / O_{F}^{\times}$such that $G^{\prime} \simeq G$ and $\mathcal{O}$ is a minimal $G^{\prime}$-order.

By Proposition 4.3.5, a minimal $G$-order is uniquely determined up to $O_{F^{-}}$ isomorphism. The proof of Proposition 4.3.5] also provides a method for writing down a minimal $G$-order explicitly. For example, if $G$ is dihedral of order $2 n$ and presented as in (4.7), then the $O_{F}$-order

$$
\mathscr{O}_{n}:=O_{F}+O_{F} u+O_{F} \eta+O_{F} u \eta,
$$

is a minimal $G$-order. Take $n=6$. Then by (4.13) and Table 4.1.

$$
\mathscr{O}_{6}=O_{F}+O_{F} i+O_{F} \varsigma(3+j) / 2+O_{F} \varsigma i(3+j) / 2 \subset\left(\frac{-1,-3}{F}\right),
$$

where $3 \varsigma^{2}=\varepsilon$. Since $O_{F(j)}=O_{F}+O_{F} \varsigma(3+j) / 2$ by Lemma 7.2.1 we may write

$$
\mathscr{O}_{6}=O_{F(j)}+i O_{F(j)} \text {. }
$$

As before, we use superscripts $\dagger$ or $\ddagger$ to distinguish the two kinds, e.g. $\mathscr{O}_{2}^{\dagger}$ denotes a minimal $D_{2}^{\dagger}$-order. Let

$$
H_{2, \infty}=\left(\frac{-1,-1}{\mathbb{Q}}\right), \quad H_{3, \infty}=\left(\frac{-1,-3}{\mathbb{Q}}\right),
$$

and $\xi \in H_{2, \infty}$ be the element in (4.11). We define

$$
\mathfrak{o}_{2}:=\mathbb{Z}[i, j]=\mathbb{Z}+\mathbb{Z} i+\mathbb{Z} j+\mathbb{Z} k \subset H_{2, \infty},
$$




$$
\begin{aligned}
\mathbb{O}_{2} & :=\mathbb{Z}+\mathbb{Z} i+\mathbb{Z} j+\mathbb{Z} \xi \subset H_{2, \infty}, \\
\mathfrak{o}_{3} & :=\mathbb{Z}[i,(1+j) / 2]=\mathbb{Z}+\mathbb{Z} i+\mathbb{Z} \frac{1+j}{2}+\mathbb{Z} \frac{i(1+j)}{2} \subset H_{3, \infty} .
\end{aligned}
$$

Then $\mathscr{O}_{n}^{\dagger}=\mathfrak{o}_{n} \otimes_{\mathbb{Z}} O_{F}$ for $n=2,3$, and $\mathcal{O}_{12}:=\mathbb{D}_{2} \otimes_{\mathbb{Z}} O_{F}$ is a minimal $A_{4}$-order. It is well-known that both $\mathbb{O}_{2}$ and $\mathfrak{o}_{3}$ are maximal orders in their respective quaternion $\mathbb{Q}$-algebras, and $\left[\mathbb{\Phi}_{2}: \mathfrak{o}_{2}\right]=2$.

By the proof of Proposition 4.3.5 if $H^{\times} / O_{F}^{\times}$contains a subgroup isomorphic to $D_{4}$ or $S_{4}$, then $2 \varepsilon \in F^{\times 2}$ and $H$ may be identified with $\left(\frac{-1,-1}{F}\right)$. Fix $\vartheta \in F^{\times}$ such that $\varepsilon=2 \vartheta^{2}$ as in Section 4.2. If $\alpha \in H$ satisfies $\alpha^{2}=-1$, then we put $\sqrt{\varepsilon \alpha}=\vartheta(1+\alpha)$ since $( \pm \vartheta(1+\alpha))^{2}=\varepsilon \alpha$. Thus

$$
\mathscr{O}_{4}=O_{F}+O_{F} i+O_{F} \sqrt{\varepsilon j}+O_{F} i \sqrt{\varepsilon j} .
$$

Straightforward calculation shows that

$$
\mathbb{O}_{24}:=O_{F}+O_{F} \sqrt{\varepsilon i}+O_{F} \sqrt{\varepsilon j}+O_{F} \xi
$$

is closed under multiplication, and hence an $O_{F}$-order. Note that $\sqrt{\varepsilon k}=(2 \vartheta-$ $\sqrt{\varepsilon j}) \xi \in \mathbb{O}_{24}$. Clearly, $\mathbb{O}_{24}$ contains the minimal $A_{4}$-order $\mathcal{O}_{12}$. Thus it is a minimal $S_{4}$-order since $\sqrt{\varepsilon i} \in \mathbb{O}_{24}$. See Definition-Lemma 5.4 for a simpler presentation of $\mathbb{O}_{24}$.

The deduction of the explicit forms of minimal $O_{F}$-orders attached to $D_{2}^{\ddagger}$ or $D_{3}^{\ddagger}$ are left to the interested reader. In Table 4.3 we list for each $G \in \mathcal{G}^{\natural}$ a representative in the unique isomorphism class of minimal $G$-orders and calculate its discriminant (see Lemmas 2.7 and 2.9). The existence of a minimal $G$-order determines $H$ uniquely up to isomorphism.

TABle 4.3. Minimal $G$-orders for $d \geq 6$

\begin{tabular}{|c|c|c|c|c|}
\hline$G$ & condition on $\varepsilon$ & $H$ & minimal $G$-order & $\mathfrak{d}(\mathcal{O})$ \\
\hline$D_{2}^{\dagger}$ & & $\left(\frac{-1,-1}{F}\right)$ & $\mathscr{O}_{2}^{\dagger}:=O_{F}[i, j]$ & $4 O_{F}$ \\
\hline$D_{2}^{\ddagger}$ & $\mathrm{N}_{F / \mathbb{Q}}(\varepsilon)=1$ & $\left(\frac{-1,-\varepsilon}{F}\right)$ & $\mathscr{O}_{2}^{\ddagger}:=O_{F}[i, j]$ & $4 O_{F}$ \\
\hline$D_{3}^{\dagger}$ & & $\left(\frac{-1,-3}{F}\right)$ & $\mathscr{O}_{3}^{\dagger}:=O_{F}[i,(1+j) / 2]$ & $3 O_{F}$ \\
\hline$D_{3}^{\ddagger}$ & $\mathrm{N}_{F / \mathbb{Q}}(\varepsilon)=1$ & $\left(\frac{-\varepsilon,-3}{F}\right)$ & $\mathscr{O}_{3}^{\ddagger}:=O_{F}[i,(1+j) / 2]$ & $3 O_{F}$ \\
\hline$D_{4}$ & $2 \varepsilon \in F^{\times 2}$ & $\left(\frac{-1,-1}{F}\right)$ & $\mathscr{O}_{4}:=O_{F}+O_{F} i+O_{F} \sqrt{\varepsilon j}+O_{F} i \sqrt{\varepsilon j}$ & $2 O_{F}$ \\
\hline$D_{6}$ & $3 \varepsilon \in F^{\times 2}$ & $\left(\frac{-1,-3}{F}\right)$ & $\mathscr{O}_{6}:=O_{F(j)}+i O_{F(j)}$ & $O_{F}$ \\
\hline$A_{4}$ & & $\left(\frac{-1,-1}{F}\right)$ & $\mathcal{O}_{12}:=O_{F}+O_{F} i+O_{F} j+O_{F} \xi$ & $2 O_{F}$ \\
\hline$S_{4}$ & $2 \varepsilon \in F^{\times 2}$ & $\left(\frac{-1,-1}{F}\right)$ & $\mathbb{O}_{24}:=O_{F}+O_{F} \sqrt{\varepsilon i}+O_{F} \sqrt{\varepsilon j}+O_{F} \xi$ & $O_{F}$ \\
\hline
\end{tabular}

We make a few observations based on Table 4.3 .

Corollary 4.4.2. The minimal $O_{F}$-orders attached to $D_{6}$ and $S_{4}$ are maximal.

Proof. Let $\mathbb{O}$ be either $\mathscr{O}_{6}$ or $\mathbb{O}_{24}$. We have $\mathfrak{d}(\mathbb{O})=O_{F}$, and hence $\mathbb{O}$ is maximal.

Corollary 4.4.3. If $H$ is not isomorphic to any of the quaternion algebras in Table 4.3. (e.g. $\left.\mathfrak{d}(H) \nmid 6 O_{F}\right)$, then $\mathcal{O}^{\star}$ is cyclic for every $O_{F}$-order $\mathcal{O} \subset H$. 
Proof. If $G:=\mathcal{O}^{\star}$ is noncyclic, then $\mathcal{O}$ contains a minimal $G$-order. Thus $H$ is necessarily isomorphic to one of the quaternion algebras listed in Table 4.3 .

Corollary 4.4.4. If $\mathcal{O}$ is the minimal $O_{F}$-order attached to a finite noncyclic subgroup $G$ of $H^{\times} / O_{F}^{\times}$, then $\mathcal{O}^{\star}=G$.

Proof. Let $G^{\prime}:=\mathcal{O}^{\star}$. Clearly, $G \subseteq G^{\prime}$, and $\mathcal{O}$ is also a minimal $G^{\prime}$-order since by definition $\mathcal{O}$ is spanned over $O_{F}$ by representatives of $G \subseteq G^{\prime}$. In particular, minimal $O_{F}$-orders attached to $G$ and $G^{\prime}$ have the same discriminants. Combining Figure 4.1 and Table 4.3, we see that this is possible only if $G=G^{\prime}$.

By Corollary 4.4.4 if $\mathcal{O}$ is a minimal $O_{F}$-order attached to a noncyclic group $G \in \mathcal{G}^{\natural}$, then the Vignéras unit index

$$
\left[\mathcal{O}^{\times}: O_{F}^{\times} \mathcal{O}^{1}\right]= \begin{cases}1 & \text { if } G \in\left\{D_{2}^{\dagger}, D_{3}^{\dagger}, A_{4}\right\}, \\ 2 & \text { if } G \in\left\{D_{2}^{\ddagger}, D_{3}^{\ddagger}, D_{4}, S_{4}, D_{6}\right\} .\end{cases}
$$

\subsection{Normalizers of minimal orders.}

Lemma 4.5.1. Let $\mathcal{O}$ be one of the minimal $G$-orders in Table 4.3. The kernel of the natural homomorphism $\varphi: \mathcal{N}(\mathcal{O}) \rightarrow \operatorname{Aut}\left(\mathcal{O}^{\star}\right)$ is given as follows

$$
\operatorname{ker}(\varphi)= \begin{cases}F^{\times} \mathcal{O}^{\times} & \text {if } \mathcal{O}^{\star} \simeq D_{2} ; \\ F^{\times}\langle j\rangle & \text { if } \mathcal{O}^{\star} \simeq D_{n} \text { with } n \in\{3,4,6\} \\ F^{\times} & \text {if } \mathcal{O}^{\star} \simeq A_{4} \text { or } S_{4} .\end{cases}
$$

Proof. Let $\tilde{v} \in \mathcal{O}^{\star}$ be a nontrivial element represented by $v \in \mathcal{O}^{\times}$, and $x \in \operatorname{ker}(\varphi) \subseteq$ $\mathcal{N}(\mathcal{O})$. Since $x \tilde{v} x^{-1}=\tilde{v}$, we have $x F(v) x^{-1}=F(v)$, so either $x v x^{-1}=v$ or $x v x^{-1}=\bar{v}$. The latter case is possible only if $\bar{v} / v \in O_{F}^{\times}$. On the other hand, $\bar{v} / v$ is a root of unity in the CM-field $F(v)$. So necessarily $\bar{v}=-v$, i.e. $\operatorname{ord}(\tilde{v})=2$. Therefore, if $\mathcal{O}^{\star}$ contains an element $\tilde{\eta}$ with $\operatorname{ord}(\tilde{\eta})>2$, then $\operatorname{ker}(\varphi) \subseteq F(\tilde{\eta})^{\times}$. In particular, if $\mathcal{O}^{\star} \simeq A_{4}$ or $S_{4}$, then there exist $\tilde{\eta}_{1}, \tilde{\eta}_{2} \in \mathcal{O}^{\star}$ such that $F\left(\tilde{\eta}_{1}\right) \neq F\left(\tilde{\eta}_{2}\right)$ and $\min \left\{\operatorname{ord}\left(\tilde{\eta}_{1}\right), \operatorname{ord}\left(\tilde{\eta}_{2}\right)\right\} \geq 3$. Hence $F^{\times} \subseteq \operatorname{ker}(\varphi) \subseteq F\left(\tilde{\eta}_{1}\right)^{\times} \cap F\left(\tilde{\eta}_{2}\right)^{\times}=F^{\times}$in this case.

Next, suppose that $\mathcal{O}=O_{F}+O_{F} i+O_{F} \eta+O_{F} i \eta$ is a minimal $D_{n}$-order with $i^{2} \in\{-1,-\varepsilon\}$ and $n=\operatorname{ord}(\tilde{\eta}) \geq 3$. Note that $F(\eta)=F(j)$ for all $\mathcal{O}$ in this case, so we have $\operatorname{ker}(\varphi) \subseteq F(j)^{\times}$. Given any $x \in \operatorname{ker}(\varphi)$, if $x i x^{-1}=i$, then $x \in F(i)^{\times} \cap F(j)^{\times}=F^{\times}$, otherwise $x i x^{-1}=-i$, and hence $x \in F^{\times} j$. It follows that $\operatorname{ker}(\varphi)=F^{\times} \cup F^{\times} j=F^{\times}\langle j\rangle$ in this case.

Lastly, suppose that $\mathcal{O}^{\star} \simeq D_{2}$. Then $\mathcal{O}=O_{F}[i, j]$ in either $\left(\frac{-1,-1}{F}\right)$ or $\left(\frac{-1,-\varepsilon}{F}\right)$. For every $x \in \operatorname{ker}(\varphi)$, we have $x i x^{-1}= \pm i$ and $x j x^{-1}= \pm j$. There exists a suitable element in $\{1, i, j, k\}$ whose product with $x$ commutes with both $i$ and $j$, and hence lies in $F^{\times}$. Therefore, $\operatorname{ker}(\varphi)=F^{\times} \mathcal{O}^{\times}$.

Since we can write down $\operatorname{Aut}(G)$ for every $G \in \mathcal{G}^{\natural}$, it is a simple exercise to work out the normalizer $\mathcal{N}(\mathcal{O})$ for each minimal $G$-order $\mathcal{O}$. For $\mathcal{O}^{\star} \simeq D_{n}$ with $n \in\{4,6\}$, the non-central elements of order 2 fall into two conjugacy classes with distinct reduced norms by Remark 4.3.4. Hence the image of $\varphi$ coincides with the inner automorphism group $\operatorname{Inn}\left(\mathcal{O}^{\star}\right)$ in this case. For a set $S \subset H^{\times}$, let $F^{\times} \mathcal{O}^{\times}\langle S\rangle$ be the subgroup of $H^{\times}$generated by $F^{\times} \mathcal{O}^{\times}$and $S$. Then

$$
\mathcal{N}\left(\mathscr{O}_{2}^{\dagger}\right)=F^{\times}\left(\mathscr{O}_{2}^{\dagger}\right)^{\times}\langle 1+i, 1+j\rangle, \quad \mathcal{N}\left(\mathscr{O}_{2}^{\ddagger}\right)=F^{\times}\left(\mathscr{O}_{2}^{\ddagger}\right)^{\times}\langle 1+i\rangle,
$$




$$
\begin{aligned}
\mathcal{N}\left(\mathscr{O}_{3}^{\dagger}\right) & =F^{\times}\left(\mathscr{O}_{3}^{\dagger}\right)^{\times}\langle j\rangle, & \mathcal{N}\left(\mathscr{O}_{3}^{\ddagger}\right)=F^{\times}\left(\mathscr{O}_{3}^{\ddagger}\right)^{\times}\langle j\rangle, \\
\mathcal{N}\left(\mathcal{O}_{12}\right) & =F^{\times} \mathcal{O}_{12}^{\times}\langle 1+i\rangle, & \\
\mathcal{N}(\mathcal{O}) & =F^{\times} \mathcal{O}^{\times} \text {if } \mathcal{O} \simeq \mathscr{O}_{4}, \mathscr{O}_{6} \text { or } \mathbb{O}_{24} . &
\end{aligned}
$$

Let us denote $\overline{\mathcal{N}}(\mathcal{O}):=\mathcal{N}(\mathcal{O}) / F^{\times} \mathcal{O}^{\times}$for any order $\mathcal{O} \subset H$. Then $\overline{\mathcal{N}}\left(\mathscr{O}_{2}^{\dagger}\right) \simeq S_{3}$, and $\overline{\mathcal{N}}\left(\mathcal{O}^{\prime}\right) \simeq \mathbb{Z} / 2 \mathbb{Z}$ if $\mathcal{O}^{\prime} \in\left\{\mathscr{O}_{2}^{\ddagger}, \mathscr{O}_{3}^{\dagger}, \mathscr{O}_{3}^{\ddagger}, \mathcal{O}_{12}\right\}$. The natural action of $\mathcal{N}(\mathcal{O})$ on the set $\mathfrak{S}(\mathcal{O})$ of maximal orders containing $\mathcal{O}$ descends to $\overline{\mathcal{N}}(\mathcal{O})$. The number of orbits is denoted by

$$
\beth(\mathcal{O}):=|\overline{\mathcal{N}}(\mathcal{O}) \backslash \mathfrak{S}(\mathcal{O})|=|\mathcal{N}(\mathcal{O}) \backslash \mathfrak{S}(\mathcal{O})| .
$$

Lemma 4.5.2. Let $\mathcal{O} \subset H$ be a minimal $G$-order with $G \in \mathcal{G}^{\natural}$, and $\mathbb{O}, \mathbb{O}^{\prime} \in \mathfrak{S}(\mathcal{O})$ be two maximal $O_{F}$-orders containing $\mathcal{O}$. If $\mathbb{O}$ and $\mathbb{O}^{\prime}$ are $H^{\times}$-conjugate, then there there exists $x \in \mathcal{N}(\mathcal{O})$ such that $\mathbb{O}^{\prime}=x \mathbb{O} x^{-1}$.

Proof. By the assumption, there there exists $y \in H^{\times}$such that $\mathbb{O}=y \mathbb{O}^{\prime} y^{-1}$. Applying Lemma 4.3.6 to the groups $\mathcal{O}^{\star} \subseteq \mathbb{O}^{\star}$ and $y \mathcal{O}^{\star} y^{-1} \subseteq \mathbb{O}^{\star}$, we see that there exists $u \in \mathbb{O}^{\times}$such that $u \mathcal{O}^{\star} u^{-1}=y \mathcal{O}^{\star} y^{-1}$, or equivalently, $u \mathcal{O} u^{-1}=y \mathcal{O} y^{-1}$. Take $x=y^{-1} u$. Then $x \in \mathcal{N}(\mathcal{O})$ and $x \mathbb{O} x^{-1}=\mathbb{O}^{\prime}$.

Therefore, if $\mathcal{O}$ is a minimal $G$-order, then $\beth(\mathcal{O})$ is the number of conjugacy classes of maximal orders containing $\mathcal{O}$.

\section{REFINED TYPE NUMBERS FOR NONCYCLIC REDUCED UNIT GROUPS: PART I}

Let $H$ be a totally definite quaternion algebra over a real quadratic field $F=$ $\mathbb{Q}(\sqrt{d})$ with square-free $d \geq 6$. The refined type number $t(G)=t(H, G)$ is defined in (1.2) and counts the number of conjugacy classes of maximal orders in $H$ with reduced unit group $G$. We compute $t(G)$ for each finite noncyclic group $G$ in Figure 4.1 starting from the groups on the top and working downward. The current section treats the cases $G \in\left\{S_{4}, D_{6}, A_{4}, D_{4}, D_{2}^{\dagger}, D_{3}^{\dagger}\right\}$. The remaining cases $G \in$ $\left\{D_{2}^{\ddagger}, D_{3}^{\ddagger}\right\}$ are postponed to the next section.

By Corollary 4.4.3, if $t(G) \neq 0$ for some $G \in \mathcal{G}^{\natural}$ as in (4.12), then we may identify $H$ with one of the quaternion $F$-algebras in Table 4.3. Assume that this is the case throughout this section. Let $\mathbb{O} \subset H$ be a maximal order with $\mathbb{O}^{\star} \simeq G$. After a suitable $H^{\times}$-conjugation, $\mathbb{O}$ contains the minimal $G$-order $\mathcal{O}$ in Table 4.3 . To compute $t(G)$, we classify the set $\mathfrak{S}(\mathcal{O})$ of maximal orders containing $\mathcal{O}$, and compute the number $\beth(\mathcal{O})$ of $\mathcal{N}(\mathcal{O})$-conjugacy classes. It then follows from Lemma 4.5 .2 that

$$
t(G)=\beth(\mathcal{O})-\sum_{G<G^{\prime}} t\left(G^{\prime}\right) .
$$

The orbits in $\mathcal{N}(\mathcal{O}) \backslash \mathfrak{S}(\mathcal{O})$ consisting of maximal orders with reduced unit groups $G^{\prime}>G$ have already been accounted for in a previous iteration. After eliminating those, we write down a representative $\mathbb{O} \in \mathfrak{S}(\mathcal{O})$ for each remaining orbit in $\mathcal{N}(\mathcal{O}) \backslash \mathfrak{S}(\mathcal{O})$. Necessarily, such an $\mathbb{O}$ is intermediate to $\mathcal{O} \subseteq \mathcal{O}^{\vee}$ (the dual lattice of $\mathcal{O})$. By construction, $\mathbb{O}^{\times}=\mathcal{O}^{\times}$. Since $\mathcal{O}$ is spanned by $\mathcal{O}^{\times}$and every $x \in \mathcal{N}(\mathbb{O})$ normalizes $\mathbb{O}^{\times}$, we have

$$
\mathcal{N}(\mathbb{O}) \subseteq \mathcal{N}(\mathcal{O}) .
$$

This provides a way to work out $\mathcal{N}(\mathbb{O})$ explicitly by applying the results of Section 4.5. We then apply (3.20) to compute $h(G)$. Recall that $\omega(H)$ denotes the number of finite primes of $F$ that are ramified in $H$. 
The computation of $t\left(S_{4}\right)$ and $t\left(D_{6}\right)$ is made easiest since minimal $O_{F}$-orders attached to $S_{4}$ and $D_{6}$ turn out to be maximal by Corollary 4.4.2.

Proposition 5.1. We have

$$
\begin{aligned}
t\left(S_{4}\right) & = \begin{cases}1 & \text { if } H=\left(\frac{-1,-1}{F}\right) \\
0 & \text { otherwise }\end{cases} \\
h\left(S_{4}\right) & =h(F) t\left(S_{4}\right) .
\end{aligned}
$$

When $2 \varepsilon \in F^{\times 2}$, every maximal order in $\left(\frac{-1,-1}{F}\right)$ with reduced unit group $S_{4}$ is conjugate to $\mathbb{O}_{24}$ in 4.21$)$, and $\mathcal{N}\left(\mathbb{O}_{24}\right)=F^{\times} \mathbb{O}_{24}^{\times}$.

Proof. The normalizer of $\mathbb{O}_{24}$ is calculated in (4.26). Suppose that $2 \varepsilon \in F^{\times 2}$ and $H=\left(\frac{-1,-1}{F}\right)=H_{2, \infty} \otimes_{\mathbb{Q}} F$, where $H_{2, \infty}=\left(\frac{-1,-1}{\mathbb{Q}}\right)$ as in (4.16). Note that 2 is ramified in $F$, and the quaternion $\mathbb{Q}$-algebra $H_{2, \infty}$ is ramified only at 2 and $\infty$. It follows that $\omega(H)=0$, and hence $h\left(S_{4}\right)=h(F) t\left(S_{4}\right)$.

Remark 5.2. Suppose that $2 \varepsilon \in F^{\times 2}$, and fix $\vartheta \in F^{\times}$such that $\varepsilon=2 \vartheta^{2}$ as in Section 4.2. From the proof of Lemma 4.3.6, $O_{F}[i, j] \subset\left(\frac{-1,-1}{F}\right)$ is the unique minimal $D_{2}^{\dagger}$-suborder of $\mathbb{O}_{24}$. On the other hand, $O_{F}[i, \vartheta(j+k)]$ is a minimal $D_{2}^{\ddagger}$ suborder of $\mathbb{O}_{24}$, and any other minimal $D_{2}^{\ddagger}$-suborder of $\mathbb{O}_{24}$ is $\mathbb{O}_{24}^{\times}$-conjugate to it. We have $O_{F}[i, \vartheta(j+k)] \not \subset O_{F}+(2 \vartheta) \mathbb{O}_{24}$ since the latter order does not contain $\vartheta(j+k)$.

Proposition 5.3. We have

$$
\begin{aligned}
t\left(D_{6}\right) & = \begin{cases}1 & \text { if } H=\left(\frac{-1,-3}{F}\right) \text { and } 3 \varepsilon \in F^{\times 2}, \\
0 & \text { otherwise; }\end{cases} \\
h\left(D_{6}\right) & =h(F) t\left(D_{6}\right) .
\end{aligned}
$$

When $3 \varepsilon \in F^{\times 2}$, every maximal order in $\left(\frac{-1,-3}{F}\right)$ with reduced unit group $D_{6}$ is conjugate to $\mathscr{O}_{6}$ in 4.15), and $\mathcal{N}\left(\mathscr{O}_{6}\right)=F^{\times} \mathscr{O}_{6}^{\times}$.

Proof. The proof of Proposition 5.1 applies, mutatis mutandis, to here as well.

We introduce two maximal orders in $\left(\frac{-1,-1}{F}\right)$ when 2 is ramified in $F$. The first order provides a simplified form of $\mathbb{O}_{24}$ when $2 \varepsilon \in F^{\times 2}$ and is also used for the calculation of $t\left(A_{4}\right)$, and the second one will be used for $t\left(D_{4}\right)$ and $t\left(D_{2}^{\dagger}\right)$.

Definition-Lemma 5.4. Suppose that $d \equiv 2,3(\bmod 4)$ so that 2 is ramified in $F$, and hence $H=\left(\frac{-1,-1}{F}\right)$ splits at all finite places of $F$.

(i) Let $\mathfrak{p}$ be the unique dyadic prime of $F, K:=F(i) \subset H$, and

$$
\begin{aligned}
& B:=\left\{\begin{array}{lll}
O_{K} & \text { if } d \equiv 2 & (\bmod 4), \\
O_{F}+\mathfrak{p} O_{K} & \text { if } d \equiv 3 & (\bmod 4),
\end{array}\right. \\
& \mathbb{O}_{12}:=B+B \xi, \quad \text { where } \xi=(1+i+j+k) / 2 \in H .
\end{aligned}
$$

Then $\mathbb{O}_{12}$ is the unique maximal order containing the minimal $A_{4}$-order $\mathcal{O}_{12} \subseteq$ $H$. If $2 \varepsilon \in F^{\times 2}$, then $\mathbb{O}_{12}$ coincides with $\mathbb{O}_{24}$ in 4.21 , and

$$
\mathbb{O}_{12}^{\star} \simeq \begin{cases}S_{4} & \text { if } 2 \varepsilon \in F^{\times 2} \\ A_{4} & \text { otherwise. }\end{cases}
$$


(ii) Let L (resp. $\mathbb{O}_{4}^{\dagger}$ ) be the following subfield (resp. $O_{F}$-order) of $H$ :

$$
\begin{aligned}
L & :=\left\{\begin{array}{lll}
F((1+\sqrt{d} i+j) / 2) \simeq F(\sqrt{-(d+1)}) & \text { if } d \equiv 2 \quad(\bmod 4), \\
F(j) \simeq F(\sqrt{-1}) & \text { if } d \equiv 3 \quad(\bmod 4),
\end{array}\right. \\
\mathbb{O}_{4}^{\dagger}: & =O_{L}+i O_{L}, \quad \text { or more explicitly } \\
\mathbb{O}_{4}^{\dagger}: & =\left\{\begin{array}{lll}
O_{F}+O_{F} i+O_{F} \frac{1+\sqrt{d} i+j}{2}+O_{F} \frac{-\sqrt{d}+i+k}{2} & \text { if } d \equiv 2 \quad(\bmod 4), \\
O_{F}+O_{F} i+O_{F} \frac{\sqrt{d}+j}{2}+O_{F} \frac{\sqrt{d} i+k}{2} & \text { if } d \equiv 3 \quad(\bmod 4) .
\end{array}\right.
\end{aligned}
$$

Then $\mathbb{O}_{4}^{\dagger}$ is a maximal order containing the minimal $D_{2}^{\dagger}$-order $\mathscr{O}_{2}^{\dagger}=O_{F}[i, j]$, and it is not $H^{\times}$-conjugate to $\mathbb{O}_{12}$ in (5.8). We have

$$
\left(\mathbb{O}_{4}^{\dagger}\right)^{\star} \simeq \begin{cases}D_{4} & \text { if } 2 \varepsilon \in F^{\times 2} \\ D_{2}^{\dagger} & \text { otherwise }\end{cases}
$$

(iii) When $2 \varepsilon \in F^{\times 2}$, the maximal orders $\mathbb{O}_{12}$ and $\mathbb{O}_{4}^{\dagger}$ are denoted as $\mathbb{O}_{24}$ and $\mathbb{O}_{8}$ respectively to emphasize their reduced unit groups. Both $\mathbb{O}_{24}$ and $\mathbb{O}_{8}$ contain the minimal $D_{4}$-order $\mathscr{O}_{4}=O_{F}[i, \sqrt{\varepsilon j}]$.

Proof of Definition-Lemma 5.4. Let $H_{2, \infty}=\left(\frac{-1,-1}{\mathbb{Q}}\right)$, the unique quaternion $\mathbb{Q}$ algebra (up to isomorphism) ramified exactly at 2 and $\infty$. We have $H=H_{2, \infty} \otimes_{\mathbb{Q}} F$, which splits at all finite places of $F$ since 2 is ramified in $F$ by the assumption.

From Table 7.1, the order $B$ is the unique $O_{F}$-order in $K$ containing $O_{F}[i]$ with $\chi\left(B, O_{F}[i]\right)=\mathfrak{p}$. Straightforward calculation shows that $\mathbb{O}_{12}=B+B \xi$ is closed under multiplication, hence an $O_{F}$-order. Since $\mathcal{O}_{12}=O_{F}[i]+O_{F}[i] \xi$, we have $\mathfrak{d}\left(\mathbb{O}_{12}\right)=\mathfrak{p}^{-2} \mathfrak{d}\left(\mathcal{O}_{12}\right)=O_{F}$ by Table 4.3. Thus, $\mathbb{O}_{12}$ is a maximal order. If $2 \varepsilon \in F^{\times 2}$, then $B=O_{F}[\sqrt{\varepsilon i}]$ by Lemma 7.1.1, which implies that $\mathbb{O}_{12}=\mathbb{O}_{24}$ and hence $\mathbb{O}_{12}^{\star} \simeq S_{4}$; otherwise $\mathbb{O}_{12}^{\star} \simeq A_{4}$ since $\mathbb{O}_{12} \supset \mathcal{O}_{12}$. It is clear from the expression of $\mathbb{O}_{24}$ in (4.21) that $\mathbb{O}_{24} \supset \mathscr{O}_{4}$.

Recall that $\mathcal{O}_{12}=\mathbb{D}_{2} \otimes_{\mathbb{Z}} O_{F}$, where $\mathbb{\Phi}_{2}$ is the maximal $\mathbb{Z}$-order in $H_{2, \infty}$ defined in (4.18). Thus $\mathcal{O}_{12} \otimes \mathbb{Z}_{\ell}$ is maximal in $H \otimes \mathbb{Q}_{\ell}$ for every prime $\ell \neq 2$ since $H_{2, \infty}$ splits at $\ell$. As both $H_{2, \infty}$ and $F$ are ramified at 2, there is a unique maximal order of $H \otimes \mathbb{Q}_{2}$ containing $\mathcal{O}_{12} \otimes \mathbb{Z}_{2}$ by Lemma 2.11 Therefore, $\mathbb{O}_{12}$ is the unique maximal order containing $\mathcal{O}_{12}$, and there is no other $O_{F}$-order intermediate to $\mathcal{O}_{12} \subset \mathbb{O}_{12}$.

For (ii), one calculates directly that $\mathbb{O}_{4}^{\dagger}=O_{L}+i O_{L}$ is closed under multiplication, hence an $O_{F}$-order. Let $B^{\prime}:=O_{F}[\sqrt{d} i+j] \subset O_{L}$ if $d \equiv 2(\bmod 4)$, and $B^{\prime}:=$ $O_{F}[j] \subset O_{L}$ if $d \equiv 3(\bmod 4)$. Then $\mathscr{O}_{2}^{\dagger}=B^{\prime}+i B^{\prime}$ and $\chi\left(O_{L}: B^{\prime}\right)=2 O_{F}$. Thus $\mathfrak{d}\left(\mathbb{O}_{4}^{\dagger}\right)=\left(2 O_{F}\right)^{-2} \mathfrak{d}\left(\mathscr{O}_{2}^{\dagger}\right)=O_{F}$ by Table 4.3. It follows that $\mathbb{O}_{4}^{\dagger}$ is a maximal order containing $\mathscr{O}_{2}^{\dagger}$. Note that $\mathbb{O}_{12} \cap F(i)=B \not O_{F}[\sqrt{-1}]$, so by symmetry, results of the same form hold true if $i$ is replaced by $j$ or $k$. On the other hand, $\mathbb{O}_{4}^{\dagger} \cap F(i)=O_{F}[i] \simeq O_{F}[\sqrt{-1}]$. Thus $\mathbb{O}_{4}^{\dagger}$ is not $H^{\times}$-conjugate to $\mathbb{O}_{12}$.

Suppose that $2 \varepsilon \in F^{\times 2}$. Clearly, $\mathscr{O}_{4} \subseteq \mathbb{O}_{4}^{\dagger}$ if $d \equiv 3(\bmod 4)$. By Lemma 7.1.1. if $d \equiv 2(\bmod 4)$, then $O_{F}[\sqrt{\varepsilon j}]=O_{F(j)}$, which has a $\mathbb{Z}$-basis $\{1, \sqrt{d}, j,(\sqrt{d}+$ $\sqrt{d} j) / 2\}$. Thus, $\mathbb{O}_{4}^{\dagger} \supset \mathscr{O}_{4}$ in this case as well. We have $\left(\mathbb{O}_{4}^{\dagger}\right)^{\star} \not S_{4}$ since $\mathbb{O}_{4}^{\dagger}$ is not conjugate to $\mathbb{O}_{12}$. Now (5.13) follows directly from Table 4.2 and Figure 4.1 . 
For each prime $p \in \mathbb{N}$, we write $\left(\frac{F}{p}\right)$ for the Artin symbol [40, p. 94]:

$$
\left(\frac{F}{p}\right)= \begin{cases}1 & \text { if } p \text { splits in } F \\ 0 & \text { if } p \text { ramifies in } F \\ -1 & \text { if } p \text { is inert in } F\end{cases}
$$

Proposition 5.5. We have

$$
\begin{aligned}
t\left(A_{4}\right) & = \begin{cases}1 & \text { if } H=\left(\frac{-1,-1}{F}\right) \text { and } 2 \varepsilon \notin F^{\times 2}, \\
0 & \text { otherwise; }\end{cases} \\
h\left(A_{4}\right) & =\left(\frac{1}{2}+\frac{1}{2}\left(\frac{F}{2}\right)+\left(\frac{F}{2}\right)^{2}\right) h(F) t\left(A_{4}\right) .
\end{aligned}
$$

Suppose that $H=\left(\frac{-1,-1}{F}\right)$ and $2 \varepsilon \notin F^{\times 2}$. We set $\mathbb{O}_{12}=B+B \xi$ as in (5.8) if $d \not \equiv 1(\bmod 4), \mathbb{O}_{12}=\mathcal{O}_{12}$ if $d \equiv 1(\bmod 8)$, and

$$
\mathbb{O}_{12}=O_{F}+O_{F} \frac{(1+\sqrt{d})-2 i+(1-\sqrt{d}) j}{4}+O_{F} j+O_{F} \xi \quad \text { if } d \equiv 5 \bmod 8 .
$$

Then every maximal order $\mathbb{O} \subset H$ with $\mathbb{O}^{\star} \simeq A_{4}$ is $H^{\times}$-conjugate to $\mathbb{O}_{12}$, and

$$
\mathcal{N}\left(\mathbb{O}_{12}\right)= \begin{cases}F^{\times} \mathbb{O}_{12}^{\times} & \text {if } d \equiv 5 \quad(\bmod 8), \\ F^{\times} \mathbb{O}_{12}^{\times}\langle 1+i\rangle & \text { otherwise. }\end{cases}
$$

Proof. Suppose that $t\left(A_{4}\right) \neq 0$, and $\mathbb{O} \subset H$ is a maximal order with $\mathbb{O}^{\star} \simeq A_{4}$. By Table 4.3, we may identify $H$ with $\left(\frac{-1,-1}{F}\right)$ in such a way that $\mathbb{O} \supseteq \mathcal{O}_{12}$. Necessarily, $2 \varepsilon \notin F^{\times 2}$, otherwise $\mathbb{O}=\mathbb{O}_{24}$, which has reduced unit group $S_{4}$.

Conversely, suppose that $H=\left(\frac{-1,-1}{F}\right)$ and $2 \varepsilon \notin F^{\times 2}$. Then $t\left(S_{4}\right)=0$, and $t\left(A_{4}\right)=\beth\left(\mathcal{O}_{12}\right)$. In other words, $\mathbb{O}^{\star} \simeq A_{4}$ for every maximal order $\mathbb{O}$ containing $\mathcal{O}_{12}=\mathbb{O}_{2} \otimes_{\mathbb{Z}} O_{F}$. According to (4.25) and (5.2),

$$
\mathcal{N}(\mathbb{O}) \subseteq \mathcal{N}\left(\mathcal{O}_{12}\right)=F^{\times} \mathcal{O}_{12}^{\times}\langle 1+i\rangle=F^{\times} \mathbb{O}^{\times}\langle 1+i\rangle .
$$

We show that $\mathcal{N}\left(\mathcal{O}_{12}\right)$ acts transitively on $\mathfrak{S}\left(\mathcal{O}_{12}\right)$ so that $t\left(A_{4}\right)=1$.

If $F$ splits at 2, then $H$ is ramified at the two dyadic primes of $F$, and $\mathcal{O}_{12}$ is already a maximal order. We have $\omega(H)=2$, and $h\left(A_{4}\right)=2 h(F)$ in this case.

In the remaining two cases, there is a unique dyadic prime $\mathfrak{p}$ of $F$, and $H$ splits at all finite primes of $F$, i.e. $\omega(H)=0$. If $F$ is ramified at 2 , then $\mathbb{O}_{12}$ in (5.8) is the unique maximal order containing $\mathcal{O}_{12}$ by Definition-Lemma 5.4. Necessarily $\mathcal{N}\left(\mathbb{O}_{12}\right)=\mathcal{N}\left(\mathcal{O}_{12}\right)$, and $h\left(A_{4}\right)=\frac{1}{2} h(F)$.

If $F$ is inert at 2, then $e_{\mathfrak{p}}\left(\mathcal{O}_{12}\right)=1$ and $\mathcal{O}_{12}$ is an Eichler order of level $\mathfrak{p}=2 O_{F}$ by Lemma 2.10, Let $\mathbb{O}$ and $\mathbb{O}^{\prime}$ be the two maximal orders containing $\mathcal{O}_{12}$. Note that $(1+i) \in \mathcal{N}\left(\mathcal{O}_{12}\right)$, but $(1+i) \notin \mathcal{N}(\mathbb{O})$ since it is odd at $\mathfrak{p}$ (see Section 2.1). Therefore, $(1+i) \mathbb{O}(1+i)^{-1}=\mathbb{O}^{\prime}$, and $\mathcal{N}(\mathbb{O})=F^{\times} \mathbb{O}^{\times}$by (5.19). It follows that $t\left(A_{4}\right)=1$ and $h\left(A_{4}\right)=h(F)$. A direct calculation shows that the dual basis of $\{1, i, j, \xi\} \subset\left(\frac{-1,-1}{F}\right)$ is

$$
\left\{\frac{1+k}{2}, \frac{-i+k}{2}, \frac{-j+k}{2},-k\right\}
$$

and the order in (5.17) is a maximal order intermediate to $\mathcal{O}_{12} \subset \mathcal{O}_{12}^{\vee}$, so it coincides with either $\mathbb{O}$ or $\mathbb{O}^{\prime}$.

Summarizing the above three cases and interpolating, we obtain (5.16). 
Similar to $\mathcal{O}_{12}=\mathbb{D}_{2} \otimes_{\mathbb{Z}} O_{F}$, the minimal $D_{3}^{\dagger}$-order $\mathscr{O}_{3}^{\dagger}$ is also obtained from the maximal $\mathbb{Z}$-order $\mathfrak{o}_{3} \subset H_{3, \infty}=\left(\frac{-1,-3}{\mathbb{Q}}\right)$ in (4.19) by extending the scalars from $\mathbb{Z}$ to $O_{F}$. By Figure 4.1, $D_{3}^{\dagger}$ is realizable as a subgroup of $D_{6}$ but not $S_{4}$.

Proposition 5.6. We have

$$
\begin{aligned}
t\left(D_{3}^{\dagger}\right) & =\left\{\begin{array}{ll}
1 & \text { if } H \simeq\left(\frac{-1,-3}{F}\right) \\
0 & \text { otherwise. }
\end{array} \text { and } 3 \varepsilon \notin F^{\times 2},\right. \\
h\left(D_{3}^{\dagger}\right) & =\left(\frac{1}{2}+\frac{1}{2}\left(\frac{F}{3}\right)+\left(\frac{F}{3}\right)^{2}\right) h(F) t\left(D_{3}^{\dagger}\right) .
\end{aligned}
$$

Suppose that $H=\left(\frac{-1,-3}{F}\right)$ and $3 \varepsilon \notin F^{\times 2}$. We set $\mathbb{O}_{6}^{\dagger}=O_{F(j)}+i O_{F(j)}$ if $d \equiv 0$ $(\bmod 3), \mathbb{O}_{6}^{\dagger}=\mathscr{O}_{3}^{\dagger}$ if $d \equiv 1(\bmod 3)$, and

$$
\mathbb{O}_{6}^{\dagger}:=O_{F}+O_{F} \frac{i+k}{2}+O_{F} \frac{1+j}{2}+O_{F} \frac{\sqrt{d} j+k}{3} \quad \text { if } d \equiv 2 \quad(\bmod 3) .
$$

Every maximal order $\mathbb{O} \subset H$ with $\mathbb{O}^{\star} \simeq D_{3}^{\dagger}$ is $H^{\times}$-conjugate to $\mathbb{O}_{6}^{\dagger}$, and

$$
\mathcal{N}\left(\mathbb{O}_{6}^{\dagger}\right)= \begin{cases}F^{\times}\left(\mathbb{O}_{6}^{\dagger}\right)^{\times} & \text {if } d \equiv 2 \quad(\bmod 3), \\ F^{\times}\left(\mathbb{O}_{6}^{\dagger}\right)^{\times}\langle j\rangle & \text { otherwise }\end{cases}
$$

Proof. By (2.7), the dual lattice of $\mathscr{O}_{3}^{\dagger}$ is

$$
\left(\mathscr{O}_{3}^{\dagger}\right)^{\vee}=O_{F} \frac{3+j}{6}+O_{F} \frac{-3 i+k}{6}+O_{F} \frac{j}{3}+O_{F} \frac{k}{3} .
$$

When $d \equiv 2(\bmod 3)$, one checks directly that $\mathscr{O}_{3}^{\dagger}+O_{F} \frac{\sqrt{d} j+k}{3} \subseteq\left(\mathscr{O}_{3}^{\dagger}\right)^{\vee}$ is a maximal order and coincides with the one in (5.22). Similar proof as that of Proposition 5.5 shows that $\mathfrak{S}\left(\mathscr{O}_{3}^{\dagger}\right)=\left\{\mathbb{O}_{6}^{\dagger}, j \mathbb{O}_{6}^{\dagger} j^{-1}\right\}$ if $d \equiv 2(\bmod 3)$, and $\mathfrak{S}\left(\mathscr{O}_{3}^{\dagger}\right)=\left\{\mathbb{O}_{6}^{\dagger}\right\}$ otherwise. The rest of the proof is almost the same, hence omitted.

Proposition 5.7. We have

$$
\begin{aligned}
t\left(D_{4}\right) & =\left\{\begin{array}{ll}
1 & \text { if } H=\left(\frac{-1,-1}{F}\right) \\
0 & \text { otherwise; }
\end{array} \text { and } 2 \varepsilon \in F^{\times 2},\right. \\
h\left(D_{4}\right) & =h(F) t\left(D_{4}\right) .
\end{aligned}
$$

In particular,

$$
t\left(D_{4}\right)=t\left(S_{4}\right) \quad \text { for all } H .
$$

Suppose that $H=\left(\frac{-1,-1}{F}\right)$ and $2 \varepsilon \in F^{\times 2}$. Every maximal order $\mathbb{O} \subset H$ with $\mathbb{O}^{\star} \simeq D_{4}$ is $H^{\times}$-conjugate to $\mathbb{O}_{8}=\mathbb{O}_{4}^{\dagger}$ in (5.11), and

$$
\mathcal{N}\left(\mathbb{O}_{8}\right)=F^{\times} \mathbb{O}_{8}^{\times} \text {. }
$$

Proof. By Table 4.3. there exists a minimal $D_{4}$-order only if $H=\left(\frac{-1,-1}{F}\right)$ and $2 \varepsilon \in F^{\times 2}$. We show that $t\left(D_{4}\right)=1$ in this case. It is shown in DefinitionLemma 5.4 that $\mathfrak{S}\left(\mathscr{O}_{4}\right) \supseteq\left\{\mathbb{O}_{24}, \mathbb{O}_{8}\right\}$, and $\mathbb{O}_{8}$ is not $H^{\times}$-conjugate to $\mathbb{O}_{24}$. Let $\mathfrak{p}=(2 \vartheta) O_{F}$ be the unique dyadic prime of $F$, where $2 \vartheta^{2}=\varepsilon$. By [6, Corollary 1.6], $\mathscr{O}_{4}=O_{F}[i, \sqrt{\varepsilon j}]$ is a Bass order since $\mathfrak{d}\left(\mathscr{O}_{4}\right)=2 O_{F}=\mathfrak{p}^{2}$ is cube-free. Note that $i^{2} \equiv(\sqrt{\varepsilon j})^{4} \equiv 1(\bmod \mathfrak{p})$. The same proof as that of Lemma 2.7 shows that $\mathscr{O}_{4}$ is maximal at every finite place of $F$ coprime to 2, and the Eichler invariant 
$e_{\mathfrak{p}}\left(\mathscr{O}_{4}\right)=0$. It then follows from Lemma 2.5 that $\mathfrak{S}\left(\mathscr{O}_{4}\right)=\left\{\mathbb{O}_{24}, \mathbb{O}_{8}\right\}$. Since $\mathbb{O}_{8}$ is not $H^{\times}$-conjugate to $\mathbb{O}_{24}$, we have $\mathbb{O}_{8}^{\star}=\mathscr{O}_{4}^{\star} \simeq D_{4}$. Therefore,

$$
F^{\times} \mathbb{O}_{8}^{\times} \subseteq \mathcal{N}\left(\mathbb{O}_{8}\right) \subseteq \mathcal{N}\left(\mathscr{O}_{4}\right)=F^{\times} \mathscr{O}_{4}^{\times}=F^{\times} \mathbb{O}_{8}^{\times} \text {. }
$$

It follows that (5.26) holds, and $h\left(D_{4}\right)=h(F) t\left(D_{4}\right)$ since $\omega(H)=0$.

Remark 5.8. The proof of Proposition 5.7 shows that $\mathbb{O}_{24}$ and $\mathbb{O}_{8}$ intersect at an Eichler order $\mathscr{E}$ of level $\mathfrak{p}=(2 \vartheta) O_{F}$, and $\mathscr{E}$ is also the unique minimal overorder of $\mathscr{O}_{4}$. It is not hard to write it down explicitly:

$$
\mathscr{E}=O_{F}[i, \sqrt{\varepsilon j}, \xi-\sqrt{\varepsilon i}]=O_{F}+O_{F} i+O_{F} \sqrt{\varepsilon j}+O_{F}(\xi-\sqrt{\varepsilon i}) .
$$

Proposition 5.9. We have

$$
\begin{aligned}
t\left(D_{2}^{\dagger}\right) & = \begin{cases}1 & \text { if } H=\left(\frac{-1,-1}{F}\right),\left(\frac{F}{2}\right)=0 \text { and } 2 \varepsilon \notin F^{\times 2}, \\
0 & \text { otherwise; }\end{cases} \\
h\left(D_{2}^{\dagger}\right) & =\frac{1}{2} h(F) t\left(D_{2}^{\dagger}\right)
\end{aligned}
$$

Suppose that $H=\left(\frac{-1,-1}{F}\right),\left(\frac{F}{2}\right)=0$ and $2 \varepsilon \notin F^{\times 2}$. Every maximal order $\mathbb{O} \subset H$ with $\mathbb{O}^{\star} \simeq D_{2}^{\dagger}$ is $H^{\times}$-conjugate to $\mathbb{O}_{4}^{\dagger}$ in (5.11), and

$$
\mathcal{N}\left(\mathbb{O}_{4}^{\dagger}\right)=F^{\times}\left(\mathbb{O}_{4}^{\dagger}\right)^{\times}\langle 1+i\rangle .
$$

Proof. We focus on the case that $H=\left(\frac{-1,-1}{F}\right)$ since $t\left(D_{2}^{\dagger}\right)=0$ otherwise. Thanks to Lemma 2.7, $\mathscr{O}_{2}^{\dagger}=O_{F}[i, j]$ is a Gorenstein order maximal at every prime $\ell \neq 2$. We study the set of maximal orders in $H$ containing $\mathscr{O}_{2}^{\dagger}$.

If $\left(\frac{F}{2}\right)=1$, then $t\left(D_{2}^{\dagger}\right)=0$. Indeed, $H$ is ramified at the two dyadic primes of $F$ in this case, and $\mathcal{O}_{12}$ is the unique maximal order containing $\mathscr{O}_{2}^{\dagger}$ with $\mathcal{O}_{12}^{\star} \simeq$ $A_{4}$. Suppose that $\left(\frac{F}{2}\right) \neq 1$ so that there is a unique dyadic prime $\mathfrak{p}$ of $F$. Then $e_{\mathfrak{p}}\left(\mathscr{O}_{2}^{\dagger}\right)=0$ by Lemma 2.7. It follows from [6, Proposition 4.1] that there is a unique minimal overorder $\mathcal{O}$ of $\mathscr{O}_{2}^{\dagger}$, and $\chi\left(\mathcal{O}, \mathscr{O}_{2}^{\dagger}\right)=\mathfrak{p}$. In particular, $\mathfrak{S}(\mathcal{O})=\mathfrak{S}\left(\mathscr{O}_{2}^{\dagger}\right)$, and $\mathcal{O}_{12} \supseteq \mathcal{O}$. Since $\chi\left(\mathcal{O}_{12}, \mathscr{O}_{2}^{\dagger}\right)=2 O_{F}$ by Table 4.3, we see that $\mathcal{O}_{12}=\mathcal{O}$ if $\left(\frac{F}{2}\right)=-1$, forcing $\mathfrak{S}\left(\mathscr{O}_{2}^{\dagger}\right)=\mathfrak{S}\left(\mathcal{O}_{12}\right)$. Therefore, $t\left(D_{2}^{\dagger}\right) \neq 0$ only if $\left(\frac{F}{2}\right)=0$, which we assume for the rest of the proof. In this case, $\mathfrak{d}(\mathcal{O})=\chi\left(\mathcal{O}, \mathscr{O}_{2}^{\dagger}\right)^{-1} \mathfrak{d}\left(\mathscr{O}_{2}^{\dagger}\right)=\mathfrak{p}^{3}$ by Table 4.3 It follows from [6. Proposition 4.1] again that $e_{\mathfrak{p}}(\mathcal{O})=0$ since $\mathfrak{d}(\mathcal{O}) \neq \mathfrak{p}$.

Note that $O_{F}[\sqrt{-1}]$ is not maximal in $F(\sqrt{-1})$ (see Table 7.1). According to Lemma 2.8, $\mathscr{O}_{2}^{\dagger}$ is not Bass, and hence $\mathcal{O}$ is not Gorenstein by [6, Proposition 1.12]. Now it follows from Lemma 2.6 that $\aleph\left(\mathscr{O}_{2}^{\dagger}\right)=\aleph(\mathcal{O})=4$. More precisely, the subtree $\mathfrak{T}\left(\mathscr{O}_{2}^{\dagger} \otimes \mathbb{Z}_{2}\right)$ of the Bruhat-Tits tree of $H \otimes \mathbb{Q}_{2}$ is a star centered at $\operatorname{Gor}(\mathcal{O}) \otimes \mathbb{Z}_{2}$ with 3 external vertices. Here $\operatorname{Gor}(\mathcal{O})$ denotes the Gorenstein saturation of $\mathcal{O}$, which is a maximal order as shown in Lemma 2.6. According to Definition-Lemma 5.4 $\mathfrak{S}\left(\mathscr{O}_{2}^{\dagger}\right) \supseteq\left\{\mathbb{O}_{12}, \mathbb{O}_{4}^{\dagger}\right\}$. By (4.23), $\mathbb{O}_{12}$ is fixed under the action of $\overline{\mathcal{N}}\left(\mathscr{O}_{2}^{\dagger}\right)$ on $\mathfrak{S}\left(\mathscr{O}_{2}^{\dagger}\right)$. We claim that $\beth\left(\mathscr{O}_{2}^{\dagger}\right)=2$, that is, $\overline{\mathcal{N}}\left(\mathscr{O}_{2}^{\dagger}\right)$ acts transitively on the set $\mathfrak{S}^{\prime}\left(\mathscr{O}_{2}^{\dagger}\right):=$ $\mathfrak{S}\left(\mathscr{O}_{2}^{\dagger}\right) \backslash\left\{\mathbb{O}_{12}\right\}$. It then follows that $\operatorname{Gor}(\mathcal{O})=\mathbb{O}_{12}$.

In fact, $\xi=(1+i+j+k) / 2 \in \mathcal{N}\left(\mathscr{O}_{2}^{\dagger}\right)$ acts transitively on $\mathfrak{S}^{\prime}\left(\mathscr{O}_{2}^{\dagger}\right)$. Otherwise, $\xi$ lies in the normalizer of one of its members, say $\mathbb{O}^{\prime}$. Then $\xi \in \mathbb{O}^{\prime}$ by Lemma 2.2. and hence $\mathbb{O}^{\prime}$ contains $\mathcal{O}_{12}=\mathscr{O}_{2}^{\dagger}+O_{F} \xi$ as well. But this contradicts the fact that $\mathbb{O}_{12}$ is the unique maximal overorder of $\mathcal{O}_{12}$. Since $\xi$ generates the only nontrivial 
normal proper subgroup of $\overline{\mathcal{N}}\left(\mathscr{O}_{2}^{\dagger}\right) \simeq S_{3}$, the action of $\overline{\mathcal{N}}\left(\mathscr{O}_{2}^{\dagger}\right)$ on $\mathfrak{S}^{\prime}\left(\mathscr{O}_{2}^{\dagger}\right)$ identifies $\overline{\mathcal{N}}\left(\mathscr{O}_{2}^{\dagger}\right)$ with the full symmetric group on $\mathfrak{S}^{\prime}\left(\mathscr{O}_{2}^{\dagger}\right)$.

Lastly, if $2 \varepsilon \in F^{\times 2}$, then the orbits $\overline{\mathcal{N}}\left(\mathscr{O}_{2}^{\dagger}\right) \backslash \mathfrak{S}\left(\mathscr{O}_{2}^{\dagger}\right)$ are represented by $\mathbb{O}_{12}=\mathbb{O}_{24}$ and $\mathbb{O}_{8}=\mathbb{O}_{4}^{\dagger}$, with $\mathbb{O}_{24}^{\star} \simeq S_{4}$ and $\mathbb{O}_{8}^{\star} \simeq D_{4}$ respectively. Thus there are no maximal orders $\mathbb{O}$ with $\mathbb{O}^{\star} \simeq D_{2}^{\dagger}$ in this case. If $2 \varepsilon \notin F^{\times 2}$, then $\mathbb{O}_{12}^{\star} \simeq A_{4}$, and $\left(\mathbb{O}_{4}^{\dagger}\right)^{\star} \simeq D_{2}^{\dagger}$ since $\mathbb{O}_{4}^{\dagger}$ is not conjugate to $\mathbb{O}_{12}$. Hence $\mathcal{N}\left(\mathbb{O}_{4}^{\dagger}\right) \subseteq \mathcal{N}\left(\mathscr{O}_{2}^{\dagger}\right)$. One verifies directly that $(1+i) \in \mathcal{N}\left(\mathbb{O}_{4}^{\dagger}\right)$, but $\xi \notin \mathcal{N}\left(\mathbb{O}_{4}^{\dagger}\right)$ as demonstrated. It follows that

$$
\mathcal{N}\left(\mathbb{O}_{4}^{\dagger}\right)=F^{\times}\left(\mathscr{O}_{2}^{\dagger}\right)^{\times}\langle 1+i\rangle=F^{\times}\left(\mathbb{O}_{4}^{\dagger}\right)^{\times}\langle 1+i\rangle .
$$

We conclude that $h\left(D_{2}^{\dagger}\right)=h(F) / 2$ since $\omega(H)=0$.

\section{REFINED TYPE NUMBERS FOR NONCYCLIC REDUCED UNIT GROUPS: PART II}

We keep the notation and assumptions of Section 5 and study the refined type numbers and class numbers for $D_{2}^{\ddagger}$ or $D_{3}^{\ddagger}$. In particular, $F=\mathbb{Q}(\sqrt{d})$ with squarefree $d \geq 6$. We assume further that $\mathrm{N}_{F / \mathbb{Q}}(\varepsilon)=1$ throughout this section, otherwise $t\left(D_{2}^{\ddagger}\right)=t\left(D_{3}^{\ddagger}\right)=0$.

6.1. Maximal orders containing $\mathscr{O}_{2}^{\ddagger}$. In this subsection, $H$ denotes the quaternion algebra $\left(\frac{-1,-\varepsilon}{F}\right)$. We first write down the finite ramified places of $H$. By Lemma 7.2.2. if $d \equiv 1(\bmod 8)$, then $\varepsilon$ is of the form $a+b \sqrt{d} \in \mathbb{Z}[\sqrt{d}]$ with $a$ odd and $b$ divisible by 4 .

Lemma 6.1.1. The quaternion algebra $H=\left(\frac{-1,-\varepsilon}{F}\right)$ splits at all finite nondyadic primes of $F$. If $d \not \equiv 1(\bmod 8)$, then $H$ splits at the unique dyadic prime of $F$ as well. When $d \equiv 1(\bmod 8), H$ is ramified at the two dyadic primes of $F$ if and only if $\varepsilon=a+b \sqrt{d}$ with $a \equiv 1(\bmod 4)$.

Proof. By Lemma 2.7 $H$ splits at all finite nondyadic primes of $F$. First suppose that $\left(\frac{F}{2}\right) \neq 1$ so that there is a unique dyadic prime $\mathfrak{p} \subset O_{F}$. Since the number of ramified places of $H$ is even, $H$ necessarily splits at $\mathfrak{p}$ as well. Hence $H$ splits at all finite primes of $F$ in this case.

Now suppose that $d \equiv 1(\bmod 8)$. We have $F_{\mathfrak{p}}=\mathbb{Q}_{2}$ for every dyadic prime $\mathfrak{p}$, and by [34, Corollary V.3.3],

$$
\mathrm{N}_{\mathbb{Q}_{2}(\sqrt{-1}) / \mathbb{Q}_{2}}\left(\mathbb{Z}_{2}[\sqrt{-1}]^{\times}\right)=\left\{u \in \mathbb{Z}_{2}^{\times} \mid u \equiv 1 \quad(\bmod 4)\right\} .
$$

It follows that the Hilbert symbol $(-1,-\varepsilon)_{\mathfrak{p}}=1$ if and only if $v_{\mathfrak{p}}(\varepsilon+1) \geq 2$, or equivalently, $a \equiv 3(\bmod 4)$.

Let $\mathscr{O}_{2}^{\ddagger}=O_{F}[i, j] \subset H$ be the minimal $D_{2}^{\ddagger}$-order in Table 4.3, Recall that $\mathcal{N}\left(\mathscr{O}_{2}^{\ddagger}\right)=F^{\times}\left(\mathscr{O}_{2}^{\ddagger}\right)^{\times}\langle 1+i\rangle$ by (4.23), so $\overline{\mathcal{N}}\left(\mathscr{O}_{2}^{\ddagger}\right)=\mathcal{N}\left(\mathscr{O}_{2}^{\ddagger}\right) / F^{\times}\left(\mathscr{O}_{2}^{\ddagger}\right)^{\times}$is a cyclic group of order 2 generated by $1+i$. By Lemma 4.5.2. two distinct maximal orders containing $\mathscr{O}_{2}^{\ddagger}$ are $H^{\times}$-conjugate if and only if they are conjugate by $1+i$. As defined in (4.27), $\beth\left(\mathscr{O}_{2}^{\ddagger}\right)=\left|\overline{\mathcal{N}}\left(\mathscr{O}_{2}^{\ddagger}\right) \backslash \mathfrak{S}\left(\mathscr{O}_{2}^{\ddagger}\right)\right|$. According to [28, 63:3], $F(j) / F$ is unramified at every dyadic prime of $F$ if and only if $-\varepsilon$ is congruent to a square modulo $4 O_{F}$. If this is the case, then $O_{F}[j]=O_{F}+2 O_{F(j)}$ by (7.9), and hence

$$
\mathbb{O}_{j}:=O_{F(j)}+i O_{F(j)}
$$

is a maximal order because $\mathfrak{d}\left(\mathbb{O}_{j}\right)=\chi\left(\mathbb{O}_{j}: \mathscr{O}_{2}^{\ddagger}\right)^{-1} \mathfrak{d}\left(\mathscr{O}_{2}^{\ddagger}\right)=\left(2 O_{F}\right)^{-2} \cdot 4 O_{F}=O_{F}$. If there exists $\varsigma \in F^{\times}$such that $\varepsilon=3 \varsigma^{2}$, then $(j / \varsigma)^{2}=-3$, and we may identify 
$H=\left(\frac{-1,-\varepsilon}{F}\right)$ with $H^{\prime}=\left(\frac{-1,-3}{F}\right)$. In such case, $F(j)=F\left(j^{\prime}\right) \simeq F(\sqrt{-3})$, which is unramified at every dyadic prime of $F$, and $\mathbb{O}_{j}$ is identified with $\mathscr{O}_{6}$ in (4.15).

Proposition 6.1.2. Write $\varepsilon=a+b \sqrt{d}$ with $a, b \in \mathbb{N}$ if $\varepsilon \in \mathbb{Z}[\sqrt{d}]$. We have the following table for $\aleph\left(\mathscr{O}_{2}^{\ddagger}\right)$ and $\beth\left(\mathscr{O}_{2}^{\ddagger}\right)$ in $H=\left(\frac{-1,-\varepsilon}{F}\right)$

\begin{tabular}{|c|c|c|c|}
\hline$d$ & $a$ & $\aleph\left(\mathscr{O}_{2}^{\ddagger}\right)$ & $\beth\left(\mathscr{O}_{2}^{\ddagger}\right)$ \\
\hline$d \equiv 1(\bmod 8)$ & $a \equiv 1(\bmod 4)$ & 1 & 1 \\
\hline$d \equiv 1(\bmod 8)$ & $a \equiv 3(\bmod 4)$ & 4 & 2 \\
\hline$d \equiv 5(\bmod 8)$ & & 2 & 1 \\
\hline$d \equiv 3(\bmod 4)$ & $a$ is even & 2 & 2 \\
\hline \multicolumn{2}{|r|}{ otherwise } & 4 & 3 \\
\hline
\end{tabular}

Proof. By Lemma 2.7, the Eichler invariant $e_{\mathfrak{p}}\left(\mathscr{O}_{2}^{\ddagger}\right)=0$ for every dyadic prime $\mathfrak{p}$ of $F$. Moreover, $\mathfrak{d}\left(\mathscr{O}_{2}^{\ddagger}\right)=4 O_{F}$, and $\mathscr{O}_{2}^{\ddagger}$ is maximal at all finite nondyadic primes of $F$. It follows that $\aleph\left(\mathscr{O}_{2}^{\ddagger}\right)=\prod_{\mathfrak{p} \mid\left(2 O_{F}\right)} \aleph_{\mathfrak{p}}\left(\mathscr{O}_{2}^{\ddagger}\right)$.

If $d \equiv 1(\bmod 8)$ and $a \equiv 1(\bmod 4)$, then $H$ is ramified at the two dyadic primes of $F$. Hence there is a unique maximal order containing $\mathscr{O}_{2}^{\ddagger}$, and it is necessarily normalized by $1+i$.

If $d \equiv 1(\bmod 8)$ and $a \equiv 3(\bmod 4)$, then $H$ splits at the two dyadic primes of $F$. By [6, Corollary 1.6], $\mathscr{O}_{2}^{\ddagger}$ is a Bass order because $\mathfrak{d}\left(\mathscr{O}_{2}^{\ddagger}\right)$ is cube-free. Then Lemma 2.5 shows that $\aleph_{\mathfrak{p}}\left(\mathscr{O}_{2}^{\ddagger}\right)=2$ for each dyadic prime $\mathfrak{p}$ of $F$, and hence $\aleph\left(\mathscr{O}_{2}^{\ddagger}\right)=$ $2 \cdot 2=4$. Since $(1+i)$ is odd at every dyadic $\mathfrak{p}$, it does not belong to $\mathcal{N}(\mathbb{O})$ for any $\mathbb{O} \in \mathfrak{S}\left(\mathscr{O}_{2}^{\ddagger}\right)$. Thus conjugation by $(1+i)$ separates $\mathfrak{S}\left(\mathscr{O}_{2}^{\ddagger}\right)$ into two pairs of maximal orders.

When $d \equiv 5(\bmod 8), \mathfrak{p}=2 O_{F}$ is the unique dyadic prime in $F$. The same line of argument as the previous case applies here and produces the desired result.

Now suppose that 2 is ramified in $F$, i.e. $d \equiv 2,3(\bmod 4)$. By Table 7.1$] O_{F}[i]$ is a proper suborder of $O_{F(i)}$. It then follows from Lemma 2.8 that $\mathscr{O}_{2}^{\ddagger}$ is a Bass order if and only if $O_{F}[\sqrt{-\varepsilon}]$ coincides with the ring of integers of $F(\sqrt{-\varepsilon})$. The latter condition holds if and only if $d \equiv 3(\bmod 4)$ and $a$ is even by Lemmas 7.2.5 and 7.2.7. Assume that this is the case so that $\mathscr{O}_{2}^{\ddagger}$ is Bass, and we apply Lemma 2.5 again to obtain $\aleph\left(\mathscr{O}_{2}^{\ddagger}\right)=2$. By Section 7.1 , we have $\chi\left(O_{F(i)}, O_{F}[i]\right)=2 O_{F}$, so

$$
\mathbb{O}_{i}:=O_{F(i)}+j O_{F(i)}
$$

is one of the two maximal orders containing $\mathscr{O}_{2}^{\ddagger}$. Furthermore,

$$
(1+i) \mathbb{O}_{i}(1+i)^{-1}=O_{F(i)}+k O_{F(i)}=i\left(O_{F(i)}+j O_{F(i)}\right)=\mathbb{O}_{i} .
$$

Hence $\overline{\mathcal{N}}\left(\mathscr{O}_{2}^{\ddagger}\right)$ acts trivially on $\mathfrak{S}\left(\mathscr{O}_{2}^{\ddagger}\right)$.

In the remaining cases, $\mathscr{O}_{2}^{\ddagger}$ is not a Bass order. Let $\mathfrak{p}$ be the unique dyadic prime of $F$. The $\mathfrak{p}$-adic completion $\left(\mathscr{O}_{2}^{\ddagger}\right)_{\mathfrak{p}}=\mathscr{O}_{2}^{\ddagger} \otimes \mathbb{Z}_{2}$ is a Gorenstein order of Eichler invariant 0 as shown in Lemma 2.7] By [6], Proposition 1.12], there exists a unique minimal overorder $\mathcal{O}$ of $\left(\mathscr{O}_{2}^{\ddagger}\right)_{\mathfrak{p}}$, and $\mathcal{O}$ is non-Gorenstein since $\left(\mathscr{O}_{2}^{\ddagger}\right)_{\mathfrak{p}}$ is not Bass. We have $\mathfrak{d}(\mathcal{O})=\mathfrak{p}^{3} O_{F_{\mathfrak{p}}}$ by [6, Proposition 4.1]. Now it follows from Lemma 2.6] that $\aleph\left(\left(\mathscr{O}_{2}^{\ddagger}\right)_{\mathfrak{p}}\right)=\aleph(\mathcal{O})=4$. In fact, $\mathfrak{T}(\mathcal{O})$ is a star centered at the Gorenstein saturation $\operatorname{Gor}(\mathcal{O})$ with 3 exterior vertices. The symmetry forces $(1+i) \in \mathcal{N}(\operatorname{Gor}(\mathcal{O}))$. To 
obtain $\beth\left(\mathscr{O}_{2}^{\ddagger}\right)=3$, it is enough to show that conjugation by $(1+i)$ acts non-trivially on $\mathfrak{S}(\mathcal{O})$.

Firstly suppose that $d \equiv 2(\bmod 4)$, so $(1+i) / \sqrt{d}$ is integral over $O_{F_{\mathfrak{p}}}$. If $(1+i) / \sqrt{d}$ acts trivially on $\mathfrak{S}(\mathcal{O})$, then $(1+i) / \sqrt{d} \in \mathbb{O}$ for every $\mathbb{O} \in \mathfrak{S}(\mathcal{O})$ by Lemma 2.2. Thus

$$
\frac{1+i}{\sqrt{d}} \in \bigcap_{\mathbb{O} \in \mathfrak{S}(\mathcal{O})} \mathbb{O}=\mathcal{O} .
$$

However, $(1+i) / \sqrt{d}$ generates the ring of integers of $F_{\mathfrak{p}}(i)$ over $O_{F_{\mathfrak{p}}}$. In light of [7, Proposition 1.11], this contradicts the fact that $\mathcal{O}$ is non-Gorenstein.

Lastly, suppose that $d \equiv 3(\bmod 4)$ and $a$ is odd. By Lemma 7.2.5 $F(j) / F$ is unramified at the dyadic prime of $F$, so the order $\mathbb{O}_{j}$ in (6.1) is a maximal order containing $\mathscr{O}_{2}^{\ddagger}$. We claim that

$$
\mathbb{O}_{k}:=(1+i) \mathbb{O}_{j}(1+i)^{-1}=O_{F(k)}+i O_{F(k)}
$$

is distinct from $\mathbb{O}_{j}$. Indeed,

$\mathbb{O}_{j} \cap F(k)=O_{F}+k\left(\left(j^{-1} O_{F(j)}\right) \cap F\right)=O_{F}+k\left(O_{F(j)} \cap F\right)=O_{F}+k O_{F} \neq O_{F(k)}$.

The proposition is proved.

Corollary 6.1.3. Let $H=\left(\frac{-1,-\varepsilon}{F}\right)$. Then $t\left(D_{2}^{\ddagger}\right)=0$ if $d=6$. For $d \geq 7$,

$$
\begin{gathered}
t\left(D_{2}^{\ddagger}\right)+t\left(D_{4}\right)+t\left(S_{4}\right)+t\left(D_{6}\right)=\beth\left(\mathscr{O}_{2}^{\ddagger}\right), \\
\text { where either } \quad t\left(D_{6}\right)=0 \quad \text { or } \quad t\left(S_{4}\right)=t\left(D_{4}\right)=0 .
\end{gathered}
$$

Particularly, if $\{2 \varepsilon, 3 \varepsilon\} \cap F^{\times 2}=\emptyset$, then

$$
t\left(D_{2}^{\ddagger}\right)=\beth\left(\mathscr{O}_{2}^{\ddagger}\right) .
$$

Proof. For every $\mathbb{O} \in \mathfrak{S}\left(\mathscr{O}_{2}^{\ddagger}\right)$, we have $\mathbb{O}^{\star} \in\left\{D_{2}^{\ddagger}, D_{4}, S_{4}, D_{6}\right\}$ by Figure 4.1. Hence (6.4) follows from (5.1), and it holds for all $d \geq 6$. Formula (6.5) holds because $2 \varepsilon$ and $3 \varepsilon$ cannot be perfect squares simultaneously in $F$ when $d \geq 7$. When $\{2 \varepsilon, 3 \varepsilon\} \cap F^{\times 2}=\emptyset$, every term other than $t\left(D_{2}^{\ddagger}\right)$ on the left side of (6.4) is zero, so (6.4) simplifies to (6.6).

Lastly, if $d=6$, then $H=\left(\frac{-1,-\varepsilon}{F}\right)$ splits at all finite primes of $F$. Hence $H \simeq$ $\left(\frac{-1,-1}{F}\right) \simeq\left(\frac{-1,-3}{F}\right)$, so $t\left(S_{4}\right)=t\left(D_{4}\right)=t\left(D_{6}\right)=1$. It follows from Proposition 6.1.2 and (6.4) that $t\left(D_{2}^{\ddagger}\right)=0$.

By Corollary 6.1.3 we may assume that $d \geq 7$ for the rest of the discussion.

Lemma 6.1.4. Assume that $F(j) / F$ is unramified at every dyadic prime of $F$ so that $\mathbb{O}_{j}$ in (6.1) is a maximal order containing $\mathscr{O}_{2}^{\ddagger}$. Then $\mathbb{O}_{j}^{*}$ is isomorphic to neither $D_{4}$ nor $S_{4}$.

Proof. Clearly, the lemma holds if $2 \varepsilon \notin F^{\times 2}$, so assume that $2 \varepsilon \in F^{\times 2}$. Suppose that $\mathbb{O}_{j}^{\star}$ is isomorphic to either $D_{4}$ or $S_{4}$. Then there exists an element $\tilde{v} \in \mathbb{O}_{j}^{\star}$ of order 4 such that $\tilde{v}^{2} \in\left(\mathscr{O}_{2}^{\ddagger}\right)^{\star}$. However, $\tilde{i} \in\left(\mathscr{O}_{2}^{\ddagger}\right)^{\star}$ is the unique element of order 2 with $\operatorname{Nr}(\tilde{i})=1$. So we must have $\tilde{v}^{2}=\tilde{i}$. This leads to a contradiction since $\mathbb{O}_{j} \cap F(i)=O_{F}[i] \not O_{F}[\sqrt{\varepsilon i}]$ by Lemma 7.1.1. 
6.1.5. We keep the notation and assumptions of Proposition 6.1.2 If $d \equiv 1$ $(\bmod 8)$ and $a \equiv 1(\bmod 4)$, then

$$
\mathbb{O}=O_{F}+O_{F} i+O_{F} j+O_{F} \frac{1+i+j+k}{2} \subset H=\left(\frac{-1,-\varepsilon}{F}\right)
$$

is the unique maximal order containing $\mathscr{O}_{2}^{\ddagger}$. Since $\omega(H)=2$ by Lemma 6.1.1, the reduced unit group $\mathbb{O}^{\star}$ cannot be isomorphic to $S_{4}, D_{4}$, nor $D_{6}$, otherwise $H$ splits at all finite primes of $F$. Therefore, we have

$$
\begin{aligned}
& \mathbb{O}^{\star} \simeq D_{2}^{\ddagger}, \quad \mathcal{N}(\mathbb{O})=F^{\times} \mathbb{O}^{\times}\langle 1+i\rangle, \quad \text { and } \\
& t\left(D_{2}^{\ddagger}\right)=1, \quad h\left(D_{2}^{\ddagger}\right)=2 h(F) .
\end{aligned}
$$

Note that $-\varepsilon$ coincides with 3 in $O_{F} / 4 O_{F} \simeq \mathbb{Z} / 4 \mathbb{Z} \times \mathbb{Z} / 4 \mathbb{Z}$ in this case. So $F(j) / F$ is ramified at both the dyadic primes of $F$.

Suppose that $d \equiv 1(\bmod 8)$ and $a \equiv 3(\bmod 4)$. Then $-\varepsilon \equiv 1\left(\bmod 4 O_{F}\right)$, and $F(j) / F$ is unramified at every dyadic prime of $F$. We leave it to the reader to check that the following are two distinct maximal orders containing $\mathscr{O}_{2}^{\ddagger}$ :

$$
\begin{aligned}
\mathbb{O}_{j}= & O_{F(j)}+i O_{F(j)}=O_{F}+O_{F} i+O_{F} \frac{1+j}{2}+O_{F} \frac{i+k}{2}, \\
\mathbb{O}= & O_{F}+O_{F} i+O_{F} \frac{(-1+\sqrt{d})+(1+\sqrt{d}) i+2 j}{4} \\
& +O_{F} \frac{(1+\sqrt{d})+(-1+\sqrt{d}) i+2 k}{4} .
\end{aligned}
$$

We have $(1+i) \mathbb{O}_{j}(1+i)^{-1}=\mathbb{O}_{k}$, which coincides with neither $\mathbb{O}_{j}$ nor $\mathbb{O}$. Since 2 splits in $F, \mathbb{O}_{j}^{\star}$ cannot be isomorphic to $S_{4}$ or $D_{4}$, and the same for $\mathbb{O}^{\star}$. It can happen that $3 \varepsilon \in F^{\times 2}$ (e.g. when $d=3 p>9$ with $\left.p \equiv 3(\bmod 8)\right)$, in which case $\left(\frac{-1,-\varepsilon}{F}\right)=\left(\frac{-1,-3}{F}\right)$, and $\mathbb{O}_{j}$ coincides with $\mathscr{O}_{6} \subseteq\left(\frac{-1,-3}{F}\right)$ in (4.14). On the other hand, $\mathbb{O}^{\star} \nsucceq D_{6}$ since $\mathbb{O}$ is not $H^{\times}$-conjugate to $\mathbb{O}_{j}$. Therefore,

$$
\begin{array}{ll}
\mathbb{O}_{j}^{\star}= \begin{cases}D_{2}^{\ddagger} & \text { if } 3 \varepsilon \notin F^{\times 2}, \\
D_{6} & \text { if } 3 \varepsilon \in F^{\times 2},\end{cases} & \mathcal{N}\left(\mathbb{O}_{j}\right)=F^{\times} \mathbb{O}_{j}^{\times} ; \\
\mathbb{O}^{\star}=D_{2}^{\ddagger}, & \mathcal{N}(\mathbb{O})=F^{\times} \mathbb{O}^{\times} .
\end{array}
$$

By Lemma 6.1.1 $\omega(H)=0$ in this case, and hence

$$
t\left(D_{2}^{\ddagger}\right)=\left\{\begin{array}{ll}
2 & \text { if } 3 \varepsilon \notin F^{\times 2}, \\
1 & \text { if } 3 \varepsilon \in F^{\times 2},
\end{array} \text { and } \quad h\left(D_{2}^{\ddagger}\right)=h(F) t\left(D_{2}^{\ddagger}\right) .\right.
$$

6.1.6. Now suppose that $d \equiv 5(\bmod 8)$ so that $\mathfrak{p}=2 O_{F}$ is the unique dyadic prime of $F$. If $F(j)$ is unramified at $\mathfrak{p}$, then $\mathfrak{S}_{(}\left(\mathscr{O}_{2}^{\ddagger}\right)=\left\{\mathbb{O}_{j}, \mathbb{O}_{k}\right\}$. Once again, it can happen that $3 \varepsilon \in F^{\times 2}$ (e.g. when $d=3 p$ with $p \equiv 7(\bmod 8)$ ), in which case $\mathbb{O}_{j}$ coincides with $\mathscr{O}_{6} \subset\left(\frac{-1,-3}{F}\right)$ as before. Moreover, (6.12) still holds for $\mathbb{O}_{j}$.

Suppose that $F(j) / F$ is ramified at $\mathfrak{p}$. Then $O_{F}[j]=O_{F(j)}$ by Lemma 7.2.3, According to Lemma 7.2.4, there are three subcases to consider.

(i) If $\varepsilon=a+b \sqrt{d} \in \mathbb{Z}[\sqrt{d}]$, then $a \equiv 1(\bmod 4)$ and $4 \mid b$. Hence $\mathbb{O}$ in (6.11) is a maximal order containing $\mathscr{O}_{2}^{\ddagger}$. 
(ii) If $\varepsilon=(a+b \sqrt{d}) / 2$ with $a$ odd and $b \equiv 1(\bmod 4)$, then

$$
\mathbb{O}=O_{F}+O_{F} i+O_{F} \frac{(-1+\sqrt{d})+2 i+2 j}{4}+O_{F} \frac{2+(-1+\sqrt{d}) i+2 k}{4}
$$

is a maximal order containing $\mathscr{O}_{2}^{\ddagger}$.

(iii) If $\varepsilon=(a+b \sqrt{d}) / 2$ with $a$ odd and $b \equiv 3(\bmod 4)$, then

$$
\mathbb{O}=O_{F}+O_{F} i+O_{F} \frac{(1+\sqrt{d})+2 i+2 j}{4}+O_{F} \frac{2+(1+\sqrt{d}) i+2 k}{4}
$$

is a maximal order containing $\mathscr{O}_{2}^{\ddagger}$.

In all three cases, we have

$$
\mathbb{O}^{\star} \simeq D_{2}^{\ddagger}, \quad \text { and } \quad \mathcal{N}(\mathbb{O})=F^{\times} \mathbb{O}^{\times} .
$$

Summarizing, if $d \equiv 5(\bmod 8)$, then $\omega(H)=0$, and

$$
t\left(D_{2}^{\ddagger}\right)=\left\{\begin{array}{ll}
1 & \text { if } 3 \varepsilon \notin F^{\times 2}, \\
0 & \text { if } 3 \varepsilon \in F^{\times 2},
\end{array} \quad \text { and } \quad h\left(D_{2}^{\ddagger}\right)=h(F) t\left(D_{2}^{\ddagger}\right) .\right.
$$

6.1.7. Next, suppose that $d \equiv 3(\bmod 4)$ and $\varepsilon=a+b \sqrt{d}$ with $a$ even. Then $F(j) / F$ is ramified at the unique dyadic prime $\mathfrak{p}$ of $F$, and $O_{F(j)}=O_{F}[j]$ by Lemma 7.2.5. We have $\mathfrak{S}\left(\mathscr{O}_{2}^{\ddagger}\right)=\left\{\mathbb{O}_{i}, \mathbb{O}\right\}$, where $\mathbb{O}_{i}$ is defined in (6.2), and $\mathbb{O}=$ $O_{F(j)}+O_{F(j)} \frac{1+i+(1+\sqrt{d}) j}{2}$. More explicitly,

$$
\mathbb{O}=O_{F}+O_{F} \frac{1+i+(1+\sqrt{d}) j}{2}+O_{F} j+O_{F} \frac{(1+\sqrt{d})+j+k}{2} .
$$

Clearly, $F(j) \not F(\sqrt{-3})$ since the latter is unramified at $\mathfrak{p}$. Hence $3 \varepsilon \notin F^{\times 2}$. On the other hand, it is possible that $2 \varepsilon \in F^{\times 2}$ (e.g. when $d=p$ with $\left.p \equiv 3(\bmod 4)\right)$. The reduced unit groups and normalizers are given by the following table

\begin{tabular}{|c|c|c|c|c|}
\hline & $\mathbb{O}_{i}^{\star}$ & $\mathcal{N}\left(\mathbb{O}_{i}\right)$ & $\mathbb{O}^{*}$ & $\mathcal{N}(\mathbb{O})$ \\
\hline $2 \varepsilon \notin F^{\times 2}$ & $D_{2}^{\ddagger}$ & $F^{\times} \mathbb{O}_{i}^{\times}\langle 1+i\rangle$ & $D_{2}^{\ddagger}$ & $F^{\times} \mathbb{O}^{\times}\langle 1+i\rangle$ \\
\hline $2 \varepsilon \in F^{\times 2}$ & $D_{4}$ & $F^{\times} \mathbb{O}_{i}^{\times}$ & $S_{4}$ & $F^{\times} \mathbb{O}^{\times}$ \\
\hline
\end{tabular}

In this case we have

$$
t\left(D_{2}^{\ddagger}\right)=\left\{\begin{array}{ll}
2 & \text { if } 2 \varepsilon \notin F^{\times 2}, \\
0 & \text { if } 2 \varepsilon \in F^{\times 2},
\end{array} \text { and } \quad h\left(D_{2}^{\ddagger}\right)=h(F) t\left(D_{2}^{\ddagger}\right) / 2 .\right.
$$

6.1.8. We treat the remaining cases where one of the following is true:

- $d \equiv 2(\bmod 4)$ and $d>6$;

- $d \equiv 3(\bmod 4)$ and $\varepsilon=a+b \sqrt{d}$ with $a$ odd.

Let $\mathfrak{p}$ be the unique dyadic prime of $F$. Then $\mathfrak{T}\left(\mathscr{O}_{2}^{\ddagger} \otimes \mathbb{Z}_{2}\right)$ is a star on which conjugation by $(1+i)$ acts as a reflection, and $\aleph\left(\mathscr{O}_{2}^{\ddagger}\right)=4$. For simplicity, let $\varrho=(1+i+j+k) / 2 \in\left(\frac{-1,-\varepsilon}{F}\right)$, and $B$ be the order of $F(i) \subset\left(\frac{-1,-\varepsilon}{F}\right)$ defined in (5.7). We claim that

$$
\mathbb{O}_{0}=B+B \varrho
$$

is the maximal order in $\mathfrak{S}\left(\mathscr{O}_{2}^{\ddagger}\right)$ corresponding to the center of the $\operatorname{star} \mathfrak{T}\left(\mathscr{O}_{2}^{\ddagger} \otimes \mathbb{Z}_{2}\right)$. Clearly, $\varrho i=-i \varrho+(-1+i)$. For any $x+y i \in B$ with $x, y \in F$, we have $y(-1+i) \in B$, and hence $\varrho(x+y i)=(x-y i) \varrho+y(-1+i) \in \mathbb{O}_{0}$. Therefore, $\mathbb{O}_{0}$ is an order containing 
$\mathscr{O}_{2}^{\ddagger}$, and it is maximal because $\chi\left(\mathbb{O}_{0}, \mathscr{O}_{2}^{\ddagger}\right)=4 O_{F}$. Let $\pi=\sqrt{d}$ if $d \equiv 2(\bmod 4)$, and $\pi=1+\sqrt{d}$ if $d \equiv 3(\bmod 4)$. Then $\mathfrak{p}=(2, \pi)$, and the norm of $(1+i) \pi / 2 \in B$ over $F$ is a p-adic unit. It follows that $\mathscr{O}_{2}^{\ddagger} \subseteq O_{F}+\mathfrak{p} \mathbb{O}_{0}$. Thus $\mathfrak{T}\left(\mathscr{O}_{2}^{\ddagger} \otimes \mathbb{Z}_{2}\right)$ is centered at $\left(\mathbb{O}_{0}\right)_{\mathfrak{p}}$. Although the expression looks similar, $\mathbb{O}_{0}$ is not isomorphic to $\mathbb{O}_{12}$ in (5.8). Indeed, if $2 \varepsilon \notin F^{\times 2}$, then $\mathbb{O}_{12}^{\star} \simeq A_{4}$, which does not contain any subgroup strictly isomorphic to $D_{2}^{\ddagger}$; if $2 \varepsilon \in F^{\times 2}$, then $\mathbb{O}_{12}=\mathbb{O}_{24}$, and $\mathbb{O}_{0} \neq \mathbb{O}_{24}$ by Remark 5.2.

First suppose that $d \equiv 3(\bmod 4)$ and $\varepsilon=a+b \sqrt{d}$ with $a$ odd. Then $\mathfrak{S}\left(\mathscr{O}_{2}^{\ddagger}\right)=$ $\left\{\mathbb{O}_{i}, \mathbb{O}_{j}, \mathbb{O}_{k}, \mathbb{O}_{0}\right\}$, and $(1+i) \mathbb{O}_{j}(1+i)^{-1}=\mathbb{O}_{k}$. Note that $2 \varepsilon \notin F^{\times 2}$ in this case, otherwise $F(j) \simeq \mathbb{Q}(\sqrt{d}, \sqrt{-2})$ is totally ramified over $\mathbb{Q}$ at 2 , contradicting to Lemma 7.2.5. On the other hand, it is possible that $3 \varepsilon \in F^{\times 2}$ (e.g. $d=3 p$ with $p \equiv 1(\bmod 4))$. The reduced unit groups and normalizers are given by the following table

\begin{tabular}{|c|c|c|c|c|c|c|}
\hline & $\mathbb{O}_{j}^{\star}$ & $\mathcal{N}\left(\mathbb{O}_{j}\right)$ & $\mathbb{O}_{0}^{\star}$ & $\mathcal{N}\left(\mathbb{O}_{0}\right)$ & $\mathbb{O}_{i}^{\star}$ & $\mathcal{N}\left(\mathbb{O}_{i}\right)$ \\
\hline $3 \varepsilon \notin F^{\times 2}$ & $D_{2}^{\ddagger}$ & \multirow{2}{*}{$F^{\times} \mathbb{O}_{j}^{\times}$} & $D_{2}^{\ddagger}$ & $F^{\times} \mathbb{O}_{0}^{\times}\langle 1+i\rangle$ & $D_{2}^{\ddagger}$ & $F^{\times} \mathbb{O}_{i}^{\times}\langle 1+i\rangle$ \\
\hline $3 \varepsilon \in F^{\times 2}$ & $D_{6}$ & & & & \\
\hline
\end{tabular}

In this case, we have

$$
t\left(D_{2}^{\ddagger}\right)=\left\{\begin{array}{ll}
3 & \text { if } 3 \varepsilon \notin F^{\times 2}, \\
2 & \text { if } 3 \varepsilon \in F^{\times 2},
\end{array} \text { and } \quad h\left(D_{2}^{\ddagger}\right)= \begin{cases}2 h(F) & \text { if } 3 \varepsilon \notin F^{\times 2} \\
h(F) & \text { if } 3 \varepsilon \in F^{\times 2}\end{cases}\right.
$$

For the remaining subsection, assume that $d \equiv 2(\bmod 4)$ and $d>6$. We then have $\varepsilon=a+b \sqrt{d}$ with $a$ odd and $b$ even. Hence

$$
\mathbb{O}_{i}^{\prime}:=O_{F}+O_{F} \frac{1+i+\sqrt{d} j}{2}+O_{F} j+O_{F} \frac{\sqrt{d}+j+k}{2}
$$

is an order containing $\mathscr{O}_{2}^{\ddagger}$. Moreover, $(1+i) \mathbb{O}_{i}^{\prime}(1+i)^{-1}=\mathbb{O}_{i}^{\prime}$. Note that $\mathbb{O}_{i}^{\prime} \neq \mathbb{O}_{0}$ since $(1+i+j+k) / 2 \notin \mathbb{O}_{i}^{\prime}$.

First suppose further that $F(j) / F$ is unramified at $\mathfrak{p}$ (see Lemma 7.2.7). Then $\mathfrak{S}\left(\mathscr{O}_{2}^{\ddagger}\right)=\left\{\mathbb{O}_{i}^{\prime}, \mathbb{O}_{0}, \mathbb{O}_{j}, \mathbb{O}_{k}\right\}$. It can happen that $2 \varepsilon \in F^{\times 2}$ (e.g. when $d=2 p$ with $p \equiv 3(\bmod 4))$ or $3 \varepsilon \in F^{\times 2}$ (e.g. when $d=78$ or 222$)$ in this case. By Lemma 6.1.4 $\mathbb{O}_{j}^{\star}$ is isomorphic to neither $D_{4}$ nor $S_{4}$. When $2 \varepsilon \in F^{\times 2}$, we must have $\left\{\mathbb{O}_{0}^{\star}, \mathbb{O}_{i}^{\prime \star}\right\}=\left\{D_{4}, S_{4}\right\}$. It has already been remarked that $\mathbb{O}_{0} \neq \mathbb{O}_{24}$. Thus $\mathbb{O}_{0}^{\star} \simeq D_{4}$ and $\mathbb{O}_{i}^{\prime \star} \simeq S_{4}$ when $2 \varepsilon \in F^{\times 2}$. The reduced unit groups and normalizers are given by the following table

\begin{tabular}{|c|c|c|c|c|c|c|}
\hline & $\mathbb{O}_{j}^{\star}$ & $\mathcal{N}\left(\mathbb{O}_{j}\right)$ & $\mathbb{O}_{0}^{\star}$ & $\mathcal{N}\left(\mathbb{O}_{0}\right)$ & $\mathbb{O}_{i}^{\prime \star}$ & $\mathcal{N}\left(\mathbb{O}_{i}^{\prime}\right)$ \\
\hline$\{2 \varepsilon, 3 \varepsilon\} \cap F^{\times 2}=\emptyset$ & $D_{2}^{\ddagger}$ & \multirow{3}{*}{$F^{\times} \mathbb{O}_{j}^{\times}$} & $D_{2}^{\ddagger}$ & $F^{\times} \mathbb{O}_{0}^{\times}\langle 1+i\rangle$ & $D_{2}^{\ddagger}$ & $F^{\times} \mathbb{O}_{i}^{\prime \times}\langle 1+i\rangle$ \\
\cline { 4 - 6 } & & $D_{6}$ & & & \\
\hline $2 \varepsilon \in F^{\times 2}$ & & $D_{4}$ & $F^{\times} \mathbb{O}_{0}^{\times}$ & $S_{4}$ & $F^{\times} \mathbb{O}_{i}^{\prime \times}$ \\
\hline
\end{tabular}

In this case, we have

$$
t\left(D_{2}^{\ddagger}\right)=\left\{\begin{array}{ll}
3 & \text { if }\{2 \varepsilon, 3 \varepsilon\} \cap F^{\times 2}=\emptyset, \\
2 & \text { if } 3 \varepsilon \in F^{\times 2}, \\
1 & \text { if } 2 \varepsilon \in F^{\times 2}
\end{array} \quad h\left(D_{2}^{\ddagger}\right)= \begin{cases}2 h(F) & \text { if }\{2 \varepsilon, 3 \varepsilon\} \cap F^{\times 2}=\emptyset, \\
h(F) & \text { otherwise. }\end{cases}\right.
$$


Lastly, suppose that $d \equiv 2(\bmod 4)$ and $F(j) / F$ is ramified at $\mathfrak{p}$. By Lemma 7.2.7. if $4 \mid b$, then $a \equiv 1(\bmod 4)$, and hence

$$
\mathbb{O}_{j}^{\prime}=O_{F}+O_{F} i+O_{F} \frac{\sqrt{d}+i+j}{2}+O_{F} \frac{1+\sqrt{d} i+k}{2}
$$

is a maximal order containing $\mathscr{O}_{2}^{\ddagger}$; if $b \equiv 2(\bmod 4)$, then $a \equiv 3(\bmod 4)$, and hence

$$
\mathbb{O}_{j}^{\prime}=O_{F}+O_{F} i+O_{F} \frac{1+\sqrt{d}+\sqrt{d} i+j}{2}+O_{F} \frac{\sqrt{d}+(1+\sqrt{d}) i+k}{2}
$$

is a maximal order containing $\mathscr{O}_{2}^{\ddagger}$. Let $\mathbb{O}_{k}^{\prime}=(1+i) \mathbb{O}_{j}^{\prime}(1+i)^{-1}$. It is straightforward to check that $\mathbb{O}_{j}^{\prime} \neq \mathbb{O}_{k}^{\prime}$ in both cases. So $\mathfrak{S}\left(\mathscr{O}_{2}^{\ddagger}\right)=\left\{\mathbb{O}_{0}, \mathbb{O}_{i}^{\prime}, \mathbb{O}_{j}^{\prime}, \mathbb{O}_{k}^{\prime}\right\}$. Since $F(\sqrt{-3}) / F$ is unramified at $\mathfrak{p}$, we have $3 \varepsilon \notin F^{\times 2}$ in this case. On the other hand, it is possible that $2 \varepsilon \in F^{\times 2}$ (e.g. when $d=2 p$ for some prime $p \equiv 1(\bmod 8)$ and $p$ not of the form $x^{2}+32 y^{2}$ for any $x, y \in \mathbb{Z}$. See [11, Corollary 24.5]). The reduced unit groups and normalizers are given by the following table

\begin{tabular}{|c|c|c|c|c|c|c|}
\hline & $\mathbb{O}_{j}^{\prime \star}$ & $\mathcal{N}\left(\mathbb{O}_{j}^{\prime}\right)$ & $\mathbb{O}_{0}^{\star}$ & $\mathcal{N}\left(\mathbb{O}_{0}\right)$ & $\mathbb{O}_{i}^{\prime \star}$ & $\mathcal{N}\left(\mathbb{O}_{i}^{\prime}\right)$ \\
\hline $2 \varepsilon \notin F^{\times 2}$ & \multirow{2}{*}{$D_{2}^{\ddagger}$} & \multirow{2}{*}{$F^{\times} \mathbb{O}_{j}^{\prime \times}$} & $D_{2}^{\ddagger}$ & $F^{\times} \mathbb{O}_{0}^{\times}\langle 1+i\rangle$ & $D_{2}^{\ddagger}$ & $F^{\times} \mathbb{O}_{i}^{\prime \times}\langle 1+i\rangle$ \\
\cline { 1 - 5 } & & $D_{4}$ & $F^{\times} \mathbb{O}_{0}^{\times}$ & $S_{4}$ & $F^{\times} \mathbb{O}_{i}^{\prime \times}$ \\
\hline
\end{tabular}

In this case, we have

$$
t\left(D_{2}^{\ddagger}\right)=\left\{\begin{array}{ll}
3 & \text { if } 2 \varepsilon \notin F^{\times 2}, \\
1 & \text { if } 2 \varepsilon \in F^{\times 2},
\end{array} \text { and } \quad h\left(D_{2}^{\ddagger}\right)= \begin{cases}2 h(F) & \text { if } 2 \varepsilon \notin F^{\times 2} \\
h(F) & \text { if } 2 \varepsilon \in F^{\times 2}\end{cases}\right.
$$

Combining (6.22), (6.24), (6.27), we see that under the assumption of Section 6.1.8 on $d$ and $\varepsilon$,

$$
h\left(D_{2}^{\ddagger}\right)= \begin{cases}2 h(F) & \text { if }\{2 \varepsilon, 3 \varepsilon\} \cap F^{\times 2}=\emptyset, \\ h(F) & \text { otherwise. }\end{cases}
$$

6.2. Maximal orders containing $\mathscr{O}_{3}^{\ddagger}$. Throughout this subsection, $H$ denotes the quaternion algebra $\left(\frac{-\varepsilon,-3}{F}\right)$. We study the maximal orders in $H$ containing the minimal $D_{3}^{\ddagger}$-order $\mathscr{O}_{3}^{\ddagger}=O_{F}\left[i, \frac{1+j}{2}\right]$. By [6, Corollary 1.6], $\mathscr{O}_{3}^{\ddagger}$ is always a Bass order since $\mathfrak{d}\left(\mathscr{O}_{3}^{\ddagger}\right)=3 O_{F}$ is cube-free. We first determine the finite ramified primes of $H=\left(\frac{-\varepsilon,-3}{F}\right)$. Write $\varepsilon=\frac{a+b \sqrt{d}}{2}$, where $a, b$ are positive integers such that $a \equiv b$ $(\bmod 2)$. If $d \equiv 1(\bmod 3)$ and $\mathrm{N}_{F / \mathbb{Q}}(\varepsilon)=1$, then $3 \mid b$, which is immediately seen by taking both sides of $a^{2}-b^{2} d=4$ modulo 3 . Therefore, $\varepsilon \equiv \pm 1\left(\bmod 3 O_{F}\right)$ in this case.

Lemma 6.2.1. Let $H=\left(\frac{-\varepsilon,-3}{F}\right)$. Then $H$ splits at all finite places of $F$ coprime to 3. If $d \not \equiv 1(\bmod 3)$, then $H$ splits at the unique prime of $F$ above 3 as well. When $d \equiv 1(\bmod 3), H$ splits at the two primes of $F$ above 3 if and only if $\varepsilon \equiv-1$ $\left(\bmod 3 O_{F}\right)$.

Proof. Since $\mathfrak{d}(H)$ divides $\mathfrak{d}\left(\mathscr{O}_{3}^{\ddagger}\right)=3 O_{F}, H$ splits at all finite places of $F$ coprime to 3 . If $F$ has a unique prime $\mathfrak{q}$ above 3 , i.e. $d \not \equiv 1(\bmod 3)$, then $H$ splits at $\mathfrak{q}$ as well because it splits at an even number of places of $F$.

Lastly, suppose that $d \equiv 1(\bmod 3)$. Let $\mathfrak{q}$ be a prime of $F$ above 3 . Then $F_{\mathfrak{q}}=$ $\mathbb{Q}_{3}$. By Hensel's lemma, the quadratic form $-\varepsilon x^{2}-3 y^{2}$ represents 1 with $x, y \in \mathbb{Q}_{3}$ 
if and only if $\varepsilon \equiv-1(\bmod 3)$. Therefore, the Hilbert symbol $(-\varepsilon,-3)_{\mathfrak{q}}=1$ (i.e. $H$ splits at $\mathfrak{q})$ if and only if $\varepsilon \equiv-1(\bmod 3)$.

Proposition 6.2.2. Let $H=\left(\frac{-\varepsilon,-3}{F}\right)$, and $\mathfrak{q}$ be a prime of $F$ above 3 . The values of $\aleph\left(\mathscr{O}_{3}^{\ddagger}\right)$ and $\beth\left(\mathscr{O}_{3}^{\ddagger}\right)$ are listed in the following table

\begin{tabular}{|c|c|c|c|}
\hline$d \geq 6$ & $\varepsilon$ & $\aleph\left(\mathscr{O}_{3}^{\ddagger}\right)$ & $\beth\left(\mathscr{O}_{3}^{\ddagger}\right)$ \\
\hline \multirow{2}{*}{$d \equiv 0(\bmod 3)$} & $\varepsilon \equiv 1(\bmod \mathfrak{q})$ & 1 & 1 \\
\cline { 2 - 4 } & $\varepsilon \equiv-1(\bmod \mathfrak{q})$ & 3 & 2 \\
\hline \multirow{2}{*}{$d \equiv 1(\bmod 3)$} & $\varepsilon \equiv 1\left(\bmod 3 O_{F}\right)$ & 1 & 1 \\
\cline { 2 - 4 } & $\varepsilon \equiv-1\left(\bmod 3 O_{F}\right)$ & 4 & 2 \\
\hline$d \equiv 2(\bmod 3)$ & & 2 & 1 \\
\hline
\end{tabular}

Proof. First suppose that $3 \mid d$ so that $\mathfrak{q}=(3, \sqrt{d})$. Since $F(j) \simeq F(\sqrt{-3})$, we have $O_{F}[(1+j) / 2]=O_{F}+\mathfrak{q} O_{F(j)}$ by Table 7.2 Hence $\mathbb{O}_{j}=O_{F(j)}+i O_{F(j)}$ is a maximal order containing $\mathscr{O}_{3}^{\ddagger}$ with $j \in \mathcal{N}\left(\mathbb{O}_{j}\right)$. By Lemma 2.9 $\mathscr{O}_{3}^{\ddagger}$ is maximal at all finite places of $F$ coprime to $\mathfrak{q}$, and

$$
e_{\mathfrak{q}}\left(\mathscr{O}_{3}^{\ddagger}\right)=\left\{\begin{array}{llll}
-1 & \text { if } & \varepsilon \equiv 1 & (\bmod \mathfrak{q}) ; \\
1 & \text { if } & \varepsilon \equiv 2 & (\bmod \mathfrak{q}) .
\end{array}\right.
$$

If $e_{\mathfrak{q}}\left(\mathscr{O}_{3}^{\ddagger}\right)=-1$, then $\aleph_{\mathfrak{q}}\left(\mathscr{O}_{3}^{\ddagger}\right)=1$ by [6, Corollary 3.2]. Thus $\mathbb{O}_{j}$ is the unique maximal order containing $\mathscr{O}_{3}^{\ddagger}$. Suppose that $e_{\mathfrak{q}}\left(\mathscr{O}_{3}^{\ddagger}\right)=1$ next. Then $\mathscr{O}_{3}^{\ddagger}$ is an Eichler order of level $3 O_{F}=\mathfrak{q}^{2}$ by [6, Corollary 2.2]. Hence $\aleph\left(\mathscr{O}_{3}^{\ddagger}\right)=3$. Let $\mathbb{O}$ and $\mathbb{O}^{\prime}$ be the remaining two maximal orders distinct from $\mathbb{O}_{j}$ that contain $\mathscr{O}_{3}^{\ddagger}$. Then $\mathbb{O} \cap F(j)=O_{F}[(1+j) / 2]$. Otherwise, we have $O_{F(j)} \subseteq \mathbb{O}$, and hence $\mathbb{O} \subseteq \mathbb{O}_{j}$, which contradicts our assumption. For simplicity, write $R=O_{F_{\mathrm{q}}}$. By [40, Theorem II.3.2], there exists an isomorphism $\mathbb{O} \otimes_{O_{F}} R \simeq M_{2}(R)$ such that $(1+j) / 2$ is identified with $\left(\begin{array}{ll}0 & 1 \\ 1 & 1\end{array}\right)$. Then $j$ is identified with $\left(\begin{array}{cc}-1 & 2 \\ 2 & 1\end{array}\right)$, which does not normalize $M_{2}(R)$. It follows that $j \notin \mathcal{N}(\mathbb{O})$. Therefore, $j \mathbb{O} j^{-1}=\mathbb{O}^{\prime}$, and hence $\beth\left(\mathscr{O}_{3}^{\ddagger}\right)=2$.

Next, suppose that $d \equiv 1(\bmod 3)$. By Lemma 6.2.1, if $\varepsilon \equiv 1\left(\bmod 3 O_{F}\right)$, then $H$ is ramified at the two places of $F$ above 3 and splits at all other finite places. Hence $\mathfrak{d}(H)=3 O_{F}=\mathfrak{d}\left(\mathscr{O}_{3}^{\ddagger}\right)$, which implies that $\mathscr{O}_{3}^{\ddagger}$ is maximal. Suppose next that $\varepsilon \equiv-1\left(\bmod 3 O_{F}\right)$. Then $H$ splits at all finite places of $F$. For any prime $\mathfrak{q}$ of $F$ above 3 , we have $\mathfrak{d}\left(\left(\mathscr{O}_{3}^{\ddagger}\right)_{\mathfrak{q}}\right)=3 O_{F_{\mathfrak{q}}}=\mathfrak{q} O_{F_{\mathfrak{q}}}$. It follows that $\left(\mathscr{O}_{3}^{\ddagger}\right)_{\mathfrak{q}}$ is an Eichler order of level $\mathfrak{q} O_{F_{\mathfrak{q}}}$, and hence $\aleph_{\mathfrak{q}}\left(\mathscr{O}_{3}^{\ddagger}\right)=2$. Therefore, $\aleph^{\aleph}\left(\mathscr{O}_{3}^{\ddagger}\right)=\prod_{\mathfrak{q} \mid 3 O_{F}} \aleph_{\mathfrak{q}}\left(\mathscr{O}_{3}^{\ddagger}\right)=2 \cdot 2=4$. By Section 2.1 $j \notin \mathcal{N}(\mathbb{O})$ for any maximal order $\mathbb{O}$ containing $\mathscr{O}_{3}^{\ddagger}$ since it is odd at $\mathfrak{q}$. Hence conjugation by $j$ acts on $\mathfrak{S}\left(\mathscr{O}_{3}^{\ddagger}\right)$ as the product of two disjoint transpositions, and $\beth\left(\mathscr{O}_{3}^{\ddagger}\right)=2$.

Lastly, the calculation of $\aleph\left(\mathscr{O}_{3}^{\ddagger}\right)$ and $\beth\left(\mathscr{O}_{3}^{\ddagger}\right)$ for $d \equiv 2(\bmod 3)$ is similar to the case $d \equiv 1(\bmod 3)$ and $\varepsilon \equiv-1\left(\bmod 3 O_{F}\right)$ above, hence omitted.

Corollary 6.2.3. Let $H=\left(\frac{-\varepsilon,-3}{F}\right)$. Then $t\left(D_{3}^{\ddagger}\right)=0$ if $d=6$. For $d \geq 7$,

$$
\begin{gathered}
t\left(D_{3}^{\ddagger}\right)+t\left(S_{4}\right)+t\left(D_{6}\right)=\beth\left(\mathscr{O}_{3}^{\ddagger}\right), \\
\text { where either } t\left(S_{4}\right)=0 \text { or } t\left(D_{6}\right)=0 .
\end{gathered}
$$


Particularly, if $\{2 \varepsilon, 3 \varepsilon\} \cap F^{\times 2}=\emptyset$, then

$$
t\left(D_{3}^{\ddagger}\right)=\beth\left(\mathscr{O}_{3}^{\ddagger}\right) .
$$

The proof is similar to that of Corollary 6.1.3, hence omitted.

6.2.4. Assume that $3 \mid d$ with $d>6$. By the proof of Proposition $6.2 .2, \mathfrak{S}\left(\mathscr{O}_{3}^{\ddagger}\right) \supseteq$ $\left\{\mathbb{O}_{j}\right\}$, and $j \in \mathcal{N}\left(\mathbb{O}_{j}\right)$. If $3 \varepsilon \in F^{\times 2}$, then $\mathbb{O}_{j}^{\star} \simeq D_{6}$ since $O_{F(j)}^{\star} \simeq \mathbb{Z} / 6 \mathbb{Z}$ in this case. For example, if $d=3 p$ with $p>3$ and $p \equiv 3(\bmod 4)$, then $3 \varepsilon \in F^{\times 2}$ by [9. Lemma $3(\mathrm{a})]$. Both cases $\varepsilon \equiv \pm 1(\bmod \mathfrak{q})$ may occur when $3 \varepsilon \in F^{\times 2}$, as shown by the examples $d=21$ and $d=33$. On the other hand, $\mathbb{O}_{j}^{\star}$ is never isomorphic to $S_{4}$. Indeed, $F(\xi) \cap \mathbb{O}_{24}=O_{F}[\xi] \neq O_{F(\xi)}$ in $\left(\frac{-1,-1}{F}\right)$ by (4.21), so $\mathbb{O}_{j} \nsucceq \mathbb{O}_{24}$. Therefore, $\mathbb{O}_{j}^{\star} \simeq D_{3}^{\ddagger}$ if $3 \varepsilon \notin F^{\times 2}$, in which case $\mathcal{N}\left(\mathbb{O}_{j}\right)=F^{\times} \mathbb{O}_{j}^{\times}\langle j\rangle$.

Let us write

$$
\varepsilon=a+b \sqrt{d} \quad \text { with } \quad a, b \in \frac{1}{2} \mathbb{Z} \quad \text { and } \quad a \equiv b \quad(\bmod \mathbb{Z}) .
$$

First assume that $a \equiv 1\left(\bmod \frac{3}{2} \mathbb{Z}\right)$ so that $\varepsilon \equiv 1(\bmod \mathfrak{q})$, then $\mathfrak{S}\left(\mathscr{O}_{3}^{\ddagger}\right)=\left\{\mathbb{O}_{j}\right\}$. It follows that

$$
t\left(D_{3}^{\ddagger}\right)=\left\{\begin{array}{ll}
1 & \text { if } 3 \varepsilon \notin F^{\times 2}, \\
0 & \text { if } 3 \varepsilon \in F^{\times 2},
\end{array} \quad \text { and } \quad h\left(D_{3}^{\ddagger}\right)=\frac{1}{2} h(F) t\left(D_{3}^{\ddagger}\right) .\right.
$$

Next, assume that $a \equiv-1\left(\bmod \frac{3}{2} \mathbb{Z}\right)$. We define

$$
\begin{gathered}
\mathbb{O}=\mathscr{O}_{3}^{\ddagger}+O_{F} \delta=O_{F}+O_{F} i+O_{F} \frac{1+j}{2}+O_{F} \delta, \quad \text { where } \\
\delta=\left\{\begin{array}{lll}
\frac{-3 i+2 j+k}{6} & \text { if } b \equiv 0 \quad\left(\bmod \frac{3}{2} \mathbb{Z}\right) ; \\
\frac{-3 i+2(1+\sqrt{d}) j+k}{6} & \text { if } b \equiv 1 \quad\left(\bmod \frac{3}{2} \mathbb{Z}\right) ; \\
\frac{-3 i+2(-1+\sqrt{d}) j+k}{6} & \text { if } b \equiv 2 \quad\left(\bmod \frac{3}{2} \mathbb{Z}\right) .
\end{array}\right.
\end{gathered}
$$

Then $\mathbb{O}$ is a maximal order containing $\mathscr{O}_{3}^{\ddagger}$ and distinct from $\mathbb{O}_{j}$. It follows from the proof of Proposition 6.2.2 that $\mathfrak{S}\left(\mathscr{O}_{3}^{\ddagger}\right)=\left\{\mathbb{O}, \mathbb{O}_{j}, j \mathbb{O} j^{-1}\right\}$.

In this case, it is possible that $2 \varepsilon \in F^{\times 2}$, as demonstrated by the example $d=66$ and $\varepsilon=65+8 \sqrt{66}$. Since $d>6$ by our assumption, $2 \varepsilon$ and $3 \varepsilon$ are not simultaneously perfect squares in $F$. The reduced unit groups and normalizers are given by the following table

\begin{tabular}{|c|c|c|c|c|}
\hline & $\mathbb{O}_{j}^{\star}$ & $\mathcal{N}\left(\mathbb{O}_{j}\right)$ & $\mathbb{O}^{\star}$ & $\mathcal{N}(\mathbb{O})$ \\
\cline { 1 - 1 }$\{2 \varepsilon, 3 \varepsilon\} \cap F^{\times 2}=\emptyset$ & \multirow{2}{*}{$D_{3}^{\ddagger}$} & $F^{\times} \mathbb{O}_{j}^{\times}\langle j\rangle$ & $D_{3}^{\ddagger}$ & \multirow{3}{*}{$F^{\times} \mathbb{O}^{\times}$} \\
\cline { 1 - 1 } & & & $S_{4}$ & \\
\cline { 1 - 1 } & $D_{6} \varepsilon \in F^{\times 2}$ & $F^{\times} \mathbb{O}_{j}^{\times}$ & $D_{3}^{\ddagger}$ & \\
\hline $3 \varepsilon \in F^{\times 2}$ & &
\end{tabular}

It follows that when $3 \mid d$ with $d>6$ and $\varepsilon \equiv-1(\bmod \mathfrak{q})$

$$
t\left(D_{3}^{\ddagger}\right)=\left\{\begin{array}{ll}
2 & \text { if }\{2 \varepsilon, 3 \varepsilon\} \cap F^{\times 2}=\emptyset, \\
1 & \text { otherwise }
\end{array} \quad h\left(D_{3}^{\ddagger}\right)= \begin{cases}3 h(F) / 2 & \text { if }\{2 \varepsilon, 3 \varepsilon\} \cap F^{\times 2}=\emptyset, \\
h(F) / 2 & \text { if } 2 \varepsilon \in F^{\times 2}, \\
h(F) & \text { if } 3 \varepsilon \in F^{\times 2} .\end{cases}\right.
$$


6.2.5. Assume that $d \equiv 1(\bmod 3)$. If $\varepsilon \equiv 1\left(\bmod 3 O_{F}\right)$, then $H$ is ramified at the two primes of $F$ above 3 , and $\mathscr{O}_{3}^{\ddagger}$ is maximal. We have

$$
t\left(D_{3}^{\ddagger}\right)=1, \quad \text { and } \quad h\left(D_{3}^{\ddagger}\right)=2 h(F) .
$$

Next, assume that $\varepsilon \equiv-1\left(\bmod 3 O_{F}\right)$ so that $\omega(H)=0$. We define

$$
\begin{aligned}
\mathbb{O} & =O_{F}+O_{F} i+O_{F} \frac{1+j}{2}+O_{F} \frac{-3 i+2 j+k}{6} ; \\
\mathbb{O}^{\prime} & =O_{F}+O_{F} i+O_{F} \frac{1+j}{2}+O_{F} \frac{-3 i+2 \sqrt{d} j+k}{6} .
\end{aligned}
$$

Then $\mathbb{O} \neq \mathbb{O}^{\prime}$ and $j \mathbb{O} j^{-1} \neq \mathbb{O}^{\prime}$. Therefore, $\mathfrak{S}\left(\mathscr{O}_{3}^{\ddagger}\right)=\left\{\mathbb{O}, \mathbb{O}^{\prime}, j \mathbb{O} j^{-1}, j \mathbb{O}^{\prime} j^{-1}\right\}$. Note that $3 \varepsilon \notin F^{\times 2}$ since $F / \mathbb{Q}$ is unramified at 3 . On the other hand, it is possible that $2 \varepsilon \in F^{\times 2}$ (e.g. $d=p$ with $p \equiv 7(\bmod 12)$ or $d=2 p$ with $\left.p \equiv 11(\bmod 12)\right)$. Suppose that this is the case. Then $\left\{\mathbb{O}^{\star}, \mathbb{O}^{\prime \star}\right\}=\left\{S_{4}, D_{3}^{\ddagger}\right\}$. Indeed, we cannot have $\mathbb{O}^{\star} \simeq \mathbb{O}^{\prime \star} \simeq S_{4}$ since $\mathbb{O}$ and $\mathbb{O}^{\prime}$ are not $H^{\times}$-conjugate. Write $\varepsilon=2 \vartheta^{2}$ and $2 \vartheta=x+y \sqrt{d} \in O_{F}$ with $x, y \in \frac{1}{2} \mathbb{Z}$ and $x \equiv y(\bmod \mathbb{Z})$. Note that either $x$ or $y$ lies in $\frac{3}{2} \mathbb{Z}$, and

$$
\left(\frac{j}{3} \pm \frac{2 \vartheta k}{3 \varepsilon}\right)^{2}=-1, \quad i\left(\frac{j}{3} \pm \frac{2 \vartheta k}{3 \varepsilon}\right)=-\left(\frac{j}{3} \pm \frac{2 \vartheta k}{3 \varepsilon}\right) i .
$$

If $y \in \frac{3}{2} \mathbb{Z}$, then $\frac{j}{3} \pm \frac{2 \vartheta k}{3 \varepsilon} \in \mathbb{O}$ for a suitable choice of sign depending on $\left(x \bmod \frac{3}{2} \mathbb{Z}\right)$.

\begin{tabular}{|c|c|c|c|c|c|}
\hline & & $\mathbb{O}^{\star}$ & $\mathcal{N}(\mathbb{O})$ & $\mathbb{O}^{\prime *}$ & $\mathcal{N}\left(\mathbb{O}^{\prime}\right)$ \\
\hline $2 \varepsilon \notin F^{\times 2}$ & & \multirow{2}{*}{$D_{3}^{\ddagger}$} & \multirow{3}{*}{$F^{\times} \mathbb{O}^{\times}$} & $D_{3}^{\ddagger}$ & \multirow{3}{*}{$F^{\times} \mathbb{O}^{\prime \times}$} \\
\hline $2 \varepsilon \in F^{\times 2}$ & $x \in \frac{3}{2} \mathbb{Z}$ & & & $S_{4}$ & \\
\hline $2 \varepsilon \in F^{\times 2}$ & $y \in \frac{3}{2} \mathbb{Z}$ & $S_{4}$ & & $D_{3}^{\ddagger}$ & \\
\hline
\end{tabular}
Hence $\mathbb{O}^{\star} \simeq S_{4}$ in this case. Similarly, if $x \in \frac{3}{2} \mathbb{Z}$, then $\frac{j}{3} \pm \frac{2 \vartheta k}{3 \varepsilon} \in \mathbb{O}^{\prime}$ for a suitable choice of sign depending on $\left(y \bmod \frac{3}{2} \mathbb{Z}\right)$. Hence $\mathbb{O}^{\prime \star} \simeq S_{4}$ in this case. The reduced unit groups and normalizers are summarized in the following table

It follows that when $d \equiv 1(\bmod 3)$ and $\varepsilon \equiv-1\left(\bmod 3 O_{F}\right)$

$$
t\left(D_{3}^{\ddagger}\right)=\left\{\begin{array}{ll}
2 & \text { if } 2 \varepsilon \notin F^{\times 2}, \\
1 & \text { if } 2 \varepsilon \in F^{\times 2}
\end{array} \quad \text { and } \quad h\left(D_{3}^{\ddagger}\right)=h(F) t\left(D_{3}^{\ddagger}\right) .\right.
$$

Corollary 6.2.6. Suppose that $d=p$ is a prime congruent to 7 modulo 12 so that $p \equiv 1(\bmod 3)$ and $2 \varepsilon \in F^{\times 2}$. Let $\mathbb{O}$ and $\mathbb{O}^{\prime}$ be the two maximal order defined in (6.38) and (6.39). If $p \equiv 7(\bmod 24)$, then $\mathbb{O}^{\star}=D_{3}^{\ddagger}$, otherwise $\mathbb{O}^{\prime \star}=D_{3}^{\ddagger}$.

Proof. Write $\varepsilon=2 \vartheta^{2}$ and $2 \vartheta=x+y \sqrt{p}$ as in Section 6.2.5. Since $p \equiv 3(\bmod 4)$ and $2 \vartheta \in O_{F}$, we have $x, y \in \mathbb{Z}$. Clearly, $\mathrm{N}_{F / \mathbb{Q}}(2 \vartheta)^{2}=\mathrm{N}_{F / \mathbb{Q}}(2 \varepsilon)=4$. On the other hand, $\mathrm{N}_{F / \mathbb{Q}}(2 \vartheta)=x^{2}-y^{2} p$ is a quadratic residue modulo $p$. It follows that $\mathrm{N}_{F / \mathbb{Q}}(2 \vartheta)=2\left(\frac{2}{p}\right)$. Reducing modulo 3 on both sides of $x^{2}-y^{2} p=2\left(\frac{2}{p}\right)$, we see that $x \equiv 0(\bmod 3)$ if and only if $\left(\frac{2}{p}\right)=1$, i.e. $p \equiv 7(\bmod 8)$. The corollary follows from the classification in Section 6.2.5.

6.2.7. Let $d \equiv 2(\bmod 3)$, and $\varepsilon=a+b \sqrt{d}$ be as in (6.32). Then either $a$ or $b$ lies in $\frac{3}{2} \mathbb{Z}$. We define

$$
\mathbb{O}=\mathscr{O}_{3}^{\ddagger}+O_{F} \delta=O_{F}+O_{F} i+O_{F} \frac{1+j}{2}+O_{F} \delta, \quad \text { where }
$$




$$
\delta=\left\{\begin{array}{llll}
\frac{-3 i+2 \sqrt{d} j+k}{6} & \text { if } a \equiv 1 & \left(\bmod \frac{3}{2} \mathbb{Z}\right) \text { and } b \equiv 0 & \left(\bmod \frac{3}{2} \mathbb{Z}\right) ; \\
\frac{-3 i+2 j+k}{6} & \text { if } a \equiv 2 & \left(\bmod \frac{3}{2} \mathbb{Z}\right) \text { and } b \equiv 0 & \left(\bmod \frac{3}{2} \mathbb{Z}\right) ; \\
\frac{-3 i+2(1+\sqrt{d}) j+k}{6} & \text { if } a \equiv 0 & \left(\bmod \frac{3}{2} \mathbb{Z}\right) \text { and } b \equiv 1 & \left(\bmod \frac{3}{2} \mathbb{Z}\right) ; \\
\frac{-3 i+2(-1+\sqrt{d}) j+k}{6} & \text { if } a \equiv 0 & \left(\bmod \frac{3}{2} \mathbb{Z}\right) \text { and } b \equiv 2 & \left(\bmod \frac{3}{2} \mathbb{Z}\right) .
\end{array}\right.
$$

Then $\left(\mathbb{O}\right.$ is the unique maximal order containing $\mathscr{O}_{3}^{\ddagger}$ up to conjugation by $j$. It is possible that $2 \varepsilon \in F^{\times 2}$ (e.g. when $d=2 p$ with $\left.p \equiv 7(\bmod 12)\right)$. On the other hand, $3 \varepsilon \notin F^{\times 2}$ since $F / \mathbb{Q}$ is unramified at 3 . We have

$$
\begin{aligned}
& \mathbb{O}^{\star} \simeq\left\{\begin{array}{ll}
D_{3}^{\ddagger} & \text { if } 2 \varepsilon \notin F^{\times 2} ; \\
S_{4} & \text { if } 2 \varepsilon \in F^{\times 2},
\end{array} \quad \text { and } \quad \mathcal{N}(\mathbb{O})=F^{\times} \mathbb{O}^{\times} ;\right. \\
& t\left(D_{3}^{\ddagger}\right)=\left\{\begin{array}{ll}
1 & \text { if } 2 \varepsilon \notin F^{\times 2} ; \\
0 & \text { if } 2 \varepsilon \in F^{\times 2},
\end{array} \quad \text { and } \quad h\left(D_{3}^{\ddagger}\right)=h(F) t\left(D_{3}^{\ddagger}\right)\right. \text {. }
\end{aligned}
$$

\section{Quadratic $O_{F}$-ORDERS in CM-FIELDS}

Let $F=\mathbb{Q}(\sqrt{d})$ be a real quadratic field with square-free $d \geq 6$, and $K / F$ be a CM-extension of $F$. Given an $O_{F}$-order $B$ in $K$, we denote $\left[B^{\times}: O_{F}^{\times}\right]$by $w(B)$, and the conductor $\chi\left(O_{K}, B\right)$ by $\mathfrak{f}_{B}$. The class number $h(B)$ can be calculated using the following formula in [38, p. 75]:

$$
h(B)=\frac{h(K) \mathrm{N}_{F / \mathbb{Q}}\left(\mathfrak{f}_{B}\right)}{\left[O_{K}^{\times}: B^{\times}\right]} \prod_{\mathfrak{p} \mid \mathfrak{f}_{B}}\left(1-\frac{1}{\mathrm{~N}_{F / \mathbb{Q}}(\mathfrak{p})}\left(\frac{K}{\mathfrak{p}}\right)\right),
$$

where $\left(\frac{K}{\mathfrak{p}}\right)$ is the Artin symbol (cf. (5.14) or see [40, p. 94]). For simplicity, we set $h(m)=h(\mathbb{Q}(\sqrt{m}))$ for any non-square $m \in \mathbb{Z}$.

The CM-extensions $K / F$ with $w\left(O_{K}\right)>1$ have been listed in (4.4). As explained in Section 3 to compute $h\left(H, C_{n}\right)$ it is necessarily to classify all $B$ in $K$ with $w(B)>1$. When $K=F(\sqrt{-1})$ or $F(\sqrt{-3})$, this is carried out in [38, Chapter 3], whose results are recalled in Section 7.1 . When $\mathrm{N}_{F / \mathbb{Q}}(\varepsilon)=1$, the orders $B$ in $F(\sqrt{-\varepsilon})$ with $B \supseteq O_{F}[\sqrt{-\varepsilon}]$ are studied in Section 7.2

7.1. Orders in $F(\sqrt{-1})$ and $F(\sqrt{-3})$. First, assume that $K=F(\sqrt{-1})$. Then by [38, Proposition 3.3] (see also [19), we have

$$
h(K)=\frac{1}{2} Q_{K / F} h(d) h(-d),
$$

where the Hasse unit index $Q_{K / F}=2$ if $2 \varepsilon \in F^{\times 2}$, and $Q_{K / F}=1$ otherwise.

Write $\mathfrak{p}$ for the unique dyadic prime of $F$ if 2 is ramified in $F$ (i.e. $d \not \equiv 1$ $(\bmod 4))$. If further $d \equiv 3(\bmod 4)$, then we give the order $B$ in (5.7) a more specialized notation:

$$
B_{1,2}:=O_{F}+\mathfrak{p} O_{K}=\mathbb{Z}\left[\sqrt{-1}, \alpha_{d}\right], \quad \text { where } \quad \alpha_{d}=(1+\sqrt{-1})(1+\sqrt{d}) / 2 .
$$

The $O_{F}$-orders $B \subseteq O_{K}$ with $w(B)>1$ are summarized in Table 7.1

Lemma 7.1.1. Suppose that there exists $\vartheta \in F^{\times}$such that $\varepsilon=2 \vartheta^{2}$. Set $\eta:=$ $\vartheta(1+\sqrt{-1}) \in K$ so that $\eta^{2}=\varepsilon \sqrt{-1}$. If $d \equiv 2(\bmod 4)$, then $O_{F}[\eta]=O_{K}$; if $d \equiv 3$ $(\bmod 4)$, then $O_{F}[\eta]=B_{1,2}$. 
Proof. Suppose first that $d \equiv 2(\bmod 4)$. Then $2 \vartheta \equiv \sqrt{d}\left(\bmod 2 O_{F}\right)$ since both sides represent the unique nontrivial nilpotent element in $O_{F} / 2 O_{F}$. Hence $\vartheta(1+$ $\sqrt{-1}) \equiv(\sqrt{d}+\sqrt{-d}) / 2\left(\bmod O_{F}[\sqrt{-1}]\right)$. By [26, Exercise II.42(b), p. 51]), we have $O_{F}[\eta]=O_{K}$. When $d \equiv 3(\bmod 4)$, the same proof as that of [45, Proposition 3.3] shows that $O_{F}[\eta]=B_{1,2}$.

TABLE 7.1. $O_{F}$-orders $B$ with $w(B)>1$ in $K=F(\sqrt{-1})$.

\begin{tabular}{|c|c|c|c|c|}
\hline$d$ & $B$ & $\mathfrak{f}_{B}$ & $w(B)$ & $h(B) / h(d)$ \\
\hline$d \equiv 1(\bmod 4)$ & $O_{K}$ & $O_{F}$ & 2 & $\frac{1}{2} h(-d)$ \\
\hline \multirow{2}{*}{$d \equiv 2(\bmod 4)$} & $O_{K}$ & $O_{F}$ & $2 Q_{K / F}$ & $\frac{1}{2} Q_{K / F} h(-d)$ \\
\cline { 2 - 5 } & $O_{F}[\sqrt{-1}]$ & $\mathfrak{p}$ & 2 & $h(-d)$ \\
\hline \multirow{3}{*}{$d \equiv 3(\bmod 4)$} & $O_{K}$ & $O_{F}$ & $2 Q_{K / F}$ & $\frac{1}{2} Q_{K / F} h(-d)$ \\
\cline { 2 - 5 } & $B_{1,2}$ & $\mathfrak{p}$ & $2 Q_{K / F}$ & $\frac{1}{2} Q_{K / F} h(-d)\left(2-\left(\frac{\mathbb{Q}(\sqrt{-d})}{2}\right)\right)$ \\
\cline { 2 - 5 } & $O_{F}[\sqrt{-1}]$ & $2 O_{F}$ & 2 & $h(-d)\left(2-\left(\frac{\mathbb{Q}(\sqrt{-d})}{2}\right)\right)$ \\
\hline
\end{tabular}

Next, assume that $K=F(\sqrt{-3})$. Then by [38, Proposition 3.4], we have

$$
h(K)=\frac{1}{2} Q_{K / F} h(d) h(-3 d),
$$

where the Hasse index $Q_{K / F}=2$ if $3 \varepsilon \in F^{\times 2}$, and $Q_{K / F}=1$ otherwise. In particular, if $3 \nmid d$, then $3 \varepsilon \notin F^{\times 2}$ and $Q_{K / F}=1$, in which case $O_{K}$ is the only $O_{F^{-}}$ order in $K$ with nontrivial reduced unit group. Suppose next that $3 \mid d$. Clearly, $w(B) \mid\left(3 Q_{K / F}\right)$ for every $O_{F}$-order $B$ in $K$. The $O_{F}$-orders $B$ with $3 \mid w(B)$ are summarized in Table 7.2 If $3 \varepsilon \notin F^{\times 2}$, this exhausts all orders $B$ in $K$ with $w(B)>1$. If $3 \varepsilon \in F^{\times 2}$ and $B \subset K$ is an $O_{F}$-order with $w(B)>1$ and $3 \nmid w(B)$, then $K=F(\sqrt{-\varepsilon}), w(B)=2$ and $B \supseteq O_{F}[\sqrt{-\varepsilon}]$. Such orders are classified in Section 7.2 ,

TABLe 7.2. $O_{F}$-orders $B$ in $K=F(\sqrt{-3})$ with $3 \mid w(B)$.

\begin{tabular}{|c|c|c|c|c|}
\hline$d$ & $B$ & $\mathfrak{f}_{B}$ & $w(B)$ & $h(B) / h(d)$ \\
\hline $3 \nmid d$ & $O_{K}$ & $O_{F}$ & 3 & $\frac{1}{2} h(-3 d)$ \\
\hline \multirow{2}{*}{$3 \mid d$} & $O_{K}$ & $O_{F}$ & $3 Q_{K / F}$ & $\frac{1}{2} Q_{K / F} h(-d / 3)$ \\
\cline { 2 - 5 } & $O_{F}\left[\zeta_{6}\right]$ & $\mathfrak{q}=(3, \sqrt{d})$ & 3 & $\frac{1}{2}\left(3-\left(\frac{\mathbb{Q}(\sqrt{-d / 3}}{3}\right)\right) h(-d / 3)$ \\
\hline
\end{tabular}

7.2. Orders in $F(\sqrt{-\varepsilon})$. Throughout this subsection, we assume that $\mathrm{N}_{F / \mathbb{Q}}(\varepsilon)=1$ and denote $F(\sqrt{-\varepsilon})$ by $L$. By [9, Lemma 3], there exists a pair of square-free positive integers $\{r, s\}$ such that $r s \in\{d, 4 d\}$ and $\{r \varepsilon, s \varepsilon\} \subset F^{\times 2}$. Hence

$$
L=\mathbb{Q}(\sqrt{d}, \sqrt{-r}, \sqrt{-s})=\mathbb{Q}(\sqrt{d}, \sqrt{-r})=\mathbb{Q}(\sqrt{d}, \sqrt{-s}) .
$$


In particular, $L / F$ is either ramified at every dyadic prime of $F$ or none. According to [38, Proposition 3.1], $\{r, s\}$ can be obtained from $\left\{\operatorname{Tr}_{F / \mathbb{Q}}(\varepsilon) \pm 2\right\}$ by stripping away all the perfect square factors, and

$$
h(L)=h(d) h(-r) h(-s) .
$$

If $3 \varepsilon \in F^{\times 2}$, then $3 \mid d$, and $L=F(\sqrt{-3})$. In this case, $O_{L}^{\times} / O_{F}^{\times}=\langle\tilde{\eta}\rangle \simeq \mathbb{Z} / 6 \mathbb{Z}$, where $\eta=\varsigma(3+\sqrt{-3}) / 2 \in O_{L}^{\times}$with $3 \varsigma^{2}=\varepsilon$ (see Table 4.1). We will show that $O_{F}[\eta]=O_{L}$ in Lemma 7.2.1. Note that $F(\sqrt{-3}) / F$ is unramified at every dyadic prime of $F$. The $O_{F}$-orders $B$ with $3 \mid w(B)$ have been covered in Table 7.2, so we focus on those with $2 \mid w(B)$, or equivalently $B \supseteq O_{F}[\sqrt{-\varepsilon}]$. By Lemma 7.2.1 below, these two classes of orders overlap only at $O_{L}$.

If $3 \varepsilon \notin F^{\times 2}$, then $\boldsymbol{\mu}(L)=\{ \pm 1\}$, and $O_{L}^{\times} / O_{F}^{\times}$is a cyclic group of order 2 generated by the image of $\sqrt{-\varepsilon}$, so any $O_{F}$-order $B \subset O_{L}$ with $w(B)>1$ contains $O_{F}[\sqrt{-\varepsilon}]$.

Let $\mathfrak{f}$ be the conductor of $O_{F}[\sqrt{-\varepsilon}] \subseteq O_{L}$. Then by [34, Proposition III.5],

$$
\begin{aligned}
\mathfrak{f}^{2} \mathfrak{d}_{O_{L} / O_{F}} & =\mathfrak{d}_{O_{F}[\sqrt{-\varepsilon}] / O_{F}}=4 O_{F}, \\
\mathfrak{d}_{O_{F}[\sqrt{-\varepsilon}] / \mathbb{Z}} & =\left(\mathfrak{d}_{O_{F} / \mathbb{Z}}\right)^{2} \mathrm{~N}_{F / \mathbb{Q}}\left(4 O_{F}\right)= \begin{cases}2^{4} d^{2} & \text { if } d \equiv 1 \quad(\bmod 4), \\
2^{8} d^{2} & \text { otherwise. }\end{cases}
\end{aligned}
$$

In particular, $L / F$ is unramified at all finite nondyadic primes of $F$. By [28, 63:3], $L / F$ is unramified at a dyadic prime $\mathfrak{p}$ if and only if $-\varepsilon$ is a square in $\left(O_{F} / 4 O_{F}\right)_{\mathfrak{p}}$. If this is the case for one (or equivalently, all) $\mathfrak{p}$, then $\mathfrak{d}_{O_{L} / O_{F}}=O_{F}$, and hence

$$
\begin{aligned}
& \mathfrak{f}=2 O_{F}, \quad O_{F}[\sqrt{-\varepsilon}]=O_{F}+2 O_{L}, \quad \text { and } \\
& h\left(O_{F}[\sqrt{-\varepsilon}]\right)= \begin{cases}4 h(L) \prod_{\mathfrak{p} \mid\left(2 O_{F}\right)}\left(1-\frac{1}{\mathrm{~N}(\mathfrak{p})}\left(\frac{L}{\mathfrak{p}}\right)\right) & \text { if } 3 \varepsilon \notin F^{\times 2}, \\
\left(2+\left(\frac{F}{2}\right)\right) h(d) h(-d / 3) & \text { if } 3 \varepsilon \in F^{\times 2} .\end{cases}
\end{aligned}
$$

When $L / F$ is ramified, it will be shown (in Lemmas 7.2.3, 7.2.5 and 7.2.7) that

$$
\mathfrak{f}= \begin{cases}(2, \sqrt{d}) & \text { if } d \equiv 2 \quad(\bmod 4) \\ O_{F} & \text { otherwise }\end{cases}
$$

If $O_{F}[\sqrt{-\varepsilon}]$ is non-maximal, then $O_{F}[\sqrt{-\varepsilon}] \subseteq O_{F}+\mathfrak{p} O_{L}$ for every dyadic prime of $F$. When $\left(\frac{F}{2}\right) \neq-1$, we have $O_{F} / \mathfrak{p} \simeq \mathbb{F}_{2}$, and hence

$$
h\left(O_{F}+\mathfrak{p} O_{L}\right)= \begin{cases}\left(2-\left(\frac{L}{\mathfrak{p}}\right)\right) h(L) & \text { if } 3 \varepsilon \notin F^{\times 2}, \\ h(d) h(-d / 3) & \text { if } 3 \varepsilon \in F^{\times 2} .\end{cases}
$$

There is a unique $O_{F}$-order of the form $O_{F}+\mathfrak{p} O_{L}$ if 2 is ramified in $F$, and two such orders if 2 splits in $F$.

Lemma 7.2.1. Suppose that there exists $\varsigma \in F^{\times}$such that $\varepsilon=3 \varsigma^{2}$. Then $O_{F}[\eta]=$ $O_{L}$, where $\eta=\varsigma(3+\sqrt{-3}) / 2 \in O_{L}^{\times}$.

Proof. Necessarily $3 \mid d$ since $F$ is ramified at 3. By Table 7.2, the conductor of $O_{F}\left[\zeta_{6}\right]$ coincides with the unique prime ideal $\mathfrak{q}$ of $O_{F}$ above 3 . As $O_{F}[\eta]$ contains both $O_{F}[\sqrt{-\varepsilon}]$ and $O_{F}\left[\zeta_{6}\right]$, the conductor of $O_{F}[\eta]$ divides both $\mathfrak{f}=2 O_{F}$ and $\mathfrak{q}$, so it must be $O_{F}$. 
The rest of this subsection is devoted to a case-by-case proof of (7.11) and working out the explicit criteria on $\varepsilon$ for $L / F$ to be ramified.

Lemma 7.2.2. Suppose that $d \equiv 1(\bmod 8)$. Then $\varepsilon$ is of the form $a+b \sqrt{d} \in \mathbb{Z}[\sqrt{d}]$ with $a$ odd and $b$ divisible by 4 . Moreover, if $a \equiv 3(\bmod 4)$, then $L / F$ is unramified at every dyadic prime of $F$; otherwise, $L / F$ is ramified at both the dyadic primes of $F$.

Proof. When $d \equiv 1(\bmod 8), O_{F}^{\times}=\mathbb{Z}[\sqrt{d}]^{\times}$by [45, Lemma 4.1]. In particular, $\varepsilon \in$ $\mathbb{Z}[\sqrt{d}]$, so we may write $\varepsilon=a+b \sqrt{d}$ with $a, b \in \mathbb{N}$ and $a^{2}-b^{2} d=1$. The first part of the lemma is obtained by taking modulo 8 on both sides of $a^{2}-b^{2} d=1$. The second part follows directly from $[28,63: 3]$ by noting that $O_{F} / 4 O_{F} \simeq \mathbb{Z} / 4 \mathbb{Z} \times \mathbb{Z} / 4 \mathbb{Z}$.

Lemma 7.2.3. Suppose that $d \equiv 1(\bmod 4)$, and $L / F$ is ramified at the dyadic primes of $F$. Then $O_{L}=O_{F}[\sqrt{-\varepsilon}]$.

Proof. Let $\{r, s\}$ be the pair of square-free positive integers in (7.5). By [9, Lemma 3], $r s=d$ since $d \equiv 1(\bmod 4)$. Hence $L / F$ is ramified at the dyadic primes of $F$ if and only if $r \equiv 1(\bmod 4)$, in which case

$$
\mathfrak{d}\left(O_{L} / \mathbb{Z}\right)=d \cdot(-4 r) \cdot(-4 s)=2^{4} d^{2}=\mathfrak{d}\left(O_{F}[\sqrt{-\varepsilon}] / \mathbb{Z}\right)
$$

by [26, Exercise II.42(f), p.52] and (7.8). Therefore, $O_{L}=O_{F}[\sqrt{-\varepsilon}]$ if $L / F$ is ramified at every dyadic prime of $F$.

Next, suppose that $d \equiv 5(\bmod 8)$. It is a classical problem of Eisenstein to characterize those $d$ such that $\varepsilon \in \mathbb{Z}[\sqrt{d}]$. If $\varepsilon \in \mathbb{Z}[\sqrt{d}]$, then we write $\varepsilon=a+b \sqrt{d}$ as before, otherwise write $\varepsilon=(a+b \sqrt{d}) / 2$ with both $a$ and $b$ odd. It has been shown [2,36] that the number of $d$ is infinite in each case.

Lemma 7.2.4. Suppose that $d \equiv 5(\bmod 8)$. Then $L=F(\sqrt{-\varepsilon})$ is ramified at $\mathfrak{p}=2 O_{F}$ if and only if one of the following conditions holds:

(i) $a \equiv 1(\bmod 4)$ and $4 \mid b$ if $\varepsilon=a+b \sqrt{d} \in \mathbb{Z}[\sqrt{d}]$;

(ii) $a \equiv 3(\bmod 4)$ if $\varepsilon=(a+b \sqrt{d}) / 2$ with both $a$ and $b$ odd. In this case, $d, a, b$ fall into one of the subcases listed below:

\begin{tabular}{|c|c|c|}
\hline$d(\bmod 16)$ & $a(\bmod 8)$ & $b(\bmod 8)$ \\
\hline \multirow{2}{*}{5} & 3 & \pm 1 \\
\cline { 2 - 3 } & 7 & \pm 3 \\
\hline \multirow{2}{*}{13} & 7 & \pm 1 \\
\cline { 2 - 3 } & 3 & \pm 3 \\
\hline
\end{tabular}

Proof. First suppose that $\varepsilon=a+b \sqrt{d} \in \mathbb{Z}[\sqrt{d}]$. Reducing both sides of $a^{2}-$ $b^{2} d=1$ modulo 8 , we find that $4 \mid b$ and $a$ is odd. If $a \equiv 3(\bmod 4)$, then $-\varepsilon \equiv 1\left(\bmod 4 O_{F}\right)$, and hence $L / F$ is unramified at $\mathfrak{p}=2 O_{F}$. On the other hand, $\left(O_{F} / 4 O_{F}\right)^{\times} \simeq \mathbb{Z} / 3 \mathbb{Z} \times(\mathbb{Z} / 2 \mathbb{Z})^{2}$. So the nontrivial element $-1 \in\left(O_{F} / 4 O_{F}\right)^{\times}$of order 2 cannot be a perfect square. Thus if $a \equiv 1(\bmod 4)$, then $L / F$ is ramified at $\mathfrak{p}$.

Next, suppose that $\varepsilon=(a+b \sqrt{d}) / 2$ with both $a$ and $b$ odd. Let $\{r, s\}$ be as in (7.5) so that $\{r \varepsilon, s \varepsilon\} \subset F^{\times 2}$. Recall that $r s=d$ as mentioned in Lemma 7.2.3. Write $r \varepsilon=\left(\frac{x+y \sqrt{d}}{2}\right)^{2}$ with both $x, y$ odd. Then $r a=\frac{x^{2}+y^{2} d}{2} \equiv 3(\bmod 4)$. Therefore $r \equiv 1(\bmod 4)$ if and only if $a \equiv 3(\bmod 4)$. Now suppose further that $d \equiv 5$ 
$(\bmod 16)$ and $b \equiv \pm 1(\bmod 8)$. Taking modulo 16 on both sides of $a^{2}-b^{2} d=4$, we get $a \equiv \pm 3(\bmod 8)$. It follows that $L / F$ is ramified at $\mathfrak{p}$ if and only if $a \equiv 3$ $(\bmod 8)$ in this case. The remaining cases are treated similarly and hence omitted.

We now study the cases where $\left(\frac{F}{2}\right)=0$, that is, $d \not \equiv 1(\bmod 4)$. Let $\mathfrak{p}$ be the unique dyadic prime of $F$.

Lemma 7.2.5. Suppose that $d \equiv 3(\bmod 4)$. Write $\varepsilon=a+b \sqrt{d}$ with $a, b \in \mathbb{N}$. If $a$ is even, then $L=F(\sqrt{-\varepsilon})$ is ramified at $\mathfrak{p}$, and $O_{L}=O_{F}[\sqrt{-\varepsilon}]$; otherwise, $L / F$ is unramified at all finite places of $F$.

Proof. Let $\{r, s\}$ be the pair of square-free positive integers as in (7.5). Clearly, $a$ and $b$ have opposite parity.

First, suppose that $a$ is even. By [9, Lemma 3(b)], $r s=4 d$, and hence $L / F$ is ramified above $\mathfrak{p}$ (In fact, $L / \mathbb{Q}$ is totally ramified above 2 ). It follows from [26, Exercise II.42(f), p. 52] that $\mathfrak{d}_{O_{L} / \mathbb{Z}}=(4 d) \cdot 4(-2 r) \cdot 4(-2 s)=2^{8} d^{2}$. Comparing with (7.8), we get $O_{L}=O_{F}[\sqrt{-\varepsilon}]$.

Next, suppose that $a$ is odd. Then $r s=d$ by [9, Lemma 3(a)]. Without lose of generality, we may assume that $r \equiv 3(\bmod 4)$ so that $\mathbb{Q}(\sqrt{-r})$ is unramified at 2. Therefore, $L=F(\sqrt{-r})$ is unramified at $\mathfrak{p}$, and thus unramified at all finite primes.

Remark 7.2.6. By [50, Theorem 1.1], we have $2 \mid a$ when $d=p$ is a prime congruent to 3 modulo 4. Thus $O_{F}[\sqrt{-\varepsilon}]=O_{L}$ in this case (see [45, Proposition 2.6]). Suppose that $d=p p^{\prime}$ is a product of primes with $p \equiv 3(\bmod 4)$ and $p^{\prime} \equiv 1$ $(\bmod 4)$. By [11, Corollary 18.6], the 2-primary subgroup of $\mathrm{Cl}\left(O_{F}\right)$ is a nontrivial cyclic group in this case, and it is a cyclic group of order 2 if either $\left(\frac{p}{p^{\prime}}\right)=-1$ or $\left(\frac{2}{p^{\prime}}\right)=-1$ (see [20, Table 1]). If $\left(\frac{p}{p^{\prime}}\right)=-1$, then $2 \mid a$. If $\left(\frac{p}{p^{\prime}}\right)=1$ and $\left(\frac{2}{p^{\prime}}\right)=-1$, then $2 \nmid a$. For the remaining case where $d=p p^{\prime}$ with $\left(\frac{p}{p^{\prime}}\right)=\left(\frac{2}{p^{\prime}}\right)=1$, we merely provide a few examples to show its complexity:

\begin{tabular}{|c|c|c|c|c||c|c|c|c|c|}
\hline$d$ & $p$ & $p^{\prime}$ & $\varepsilon$ & $h(F)$ & $d$ & $p$ & $p^{\prime}$ & $\varepsilon$ & $h(F)$ \\
\hline 323 & 19 & 17 & $18+\sqrt{323}$ & 4 & 799 & 47 & 17 & $424+15 \sqrt{799}$ & 8 \\
\hline 2419 & 59 & 41 & $2951+60 \sqrt{2419}$ & 12 & 943 & 23 & 41 & $737+24 \sqrt{943}$ & 4 \\
\hline
\end{tabular}

Lemma 7.2.7. Suppose that $d \equiv 2(\bmod 4)$. Then $\varepsilon=a+b \sqrt{d}$ with $a$ odd and $b$ even, and $L / F$ is ramified at $\mathfrak{p}$ if and only if the following is true:

$$
(a \bmod 4) \equiv\left\{\begin{array}{lll}
1 & \text { if } b \equiv 0 & (\bmod 4) \\
3 & \text { if } b \equiv 2 & (\bmod 4)
\end{array}\right.
$$

If $L / F$ is ramified at $\mathfrak{p}$, then $O_{F}[\sqrt{-\varepsilon}]=O_{F}+\mathfrak{p} O_{L}$.

Proof. The parity of $a$ and $b$ follows directly from $\mathrm{N}_{F / \mathbb{Q}}(\varepsilon)=a^{2}-b^{2} d=1$. Let $\{r, s\}$ be as in (7.5). By [9, Lemma $3(\mathrm{a})], r s=d$. Since $d \equiv 2(\bmod 4)$, we assume that $r$ is odd and $s$ is even. Then $L / F$ is ramified at $\mathfrak{p}$ if and only if $r \equiv 1(\bmod 4)$.

Write $r \varepsilon=(x+y \sqrt{d})^{2}$ with $x, y \in \mathbb{N}$. Then

$$
r a=x^{2}+d y^{2} \quad \text { and } \quad r b=2 x y .
$$


Necessarily, $x$ is odd. If $b \equiv 2(\bmod 4)$, then $y$ is odd, and hence $r a \equiv 3(\bmod 4)$. Thus $r \equiv 1(\bmod 4)$ if and only if $a \equiv 3(\bmod 4)$. If $b \equiv 0(\bmod 4)$, then $y$ is even, and hence $r a \equiv 1(\bmod 4)$. Thus $r \equiv 1(\bmod 4)$ if and only if $a \equiv 1(\bmod 4)$.

If $L / F$ is unramified at $\mathfrak{p}$, then $O_{F}[\sqrt{-\varepsilon}]=O_{F}+2 O_{L}$ by (7.9). Suppose that $r \equiv 1(\bmod 4)$ so that $L / F$ is ramified at $\mathfrak{p}$. Then

$$
\sqrt{-\varepsilon}=\frac{1}{r} \sqrt{-r} \sqrt{r \varepsilon} \in(\mathbb{Q} \sqrt{-r}+\mathbb{Q} \sqrt{-s}) \cap O_{L}=\mathbb{Z} \sqrt{-r}+\mathbb{Z} \sqrt{-s} .
$$

Hence $O_{F}[\sqrt{-\varepsilon}] \subseteq \mathbb{Z}[\sqrt{-r}, \sqrt{-s}]$. One calculates that $\mathfrak{d}_{\mathbb{Z}[\sqrt{-r}, \sqrt{-s}] / \mathbb{Z}}=2^{8} d^{2}=$ $\mathfrak{d}_{O_{F}[\sqrt{-\varepsilon}] / \mathbb{Z}}$ by (7.8). Therefore, $O_{F}[\sqrt{-\varepsilon}]=\mathbb{Z}[\sqrt{-r}, \sqrt{-s}]$, which has index 2 in $O_{L}$ by [26, Exercise II.42(b), p. 51]. We conclude that $O_{F}[\sqrt{-\varepsilon}]=O_{F}+\mathfrak{p} O_{L}$.

Remark 7.2.8. Suppose that $d=2 p$ with $p$ prime. If $p \equiv 3(\bmod 4)$, then $\mathrm{N}_{F / \mathbb{Q}}(\varepsilon)=1$ by [16, (V.1.7)]. Necessarily, $r=p$ in this case, so $L / F$ is unramified above $\mathfrak{p}$ by Lemma 7.2.7 If $p \equiv 5(\bmod 8)$, then $\mathrm{N}_{F / \mathbb{Q}}(\varepsilon)=-1$ by [1], Proposition 19.9]. The sign of $\mathrm{N}_{F / \mathbb{Q}}(\varepsilon)$ for $p \equiv 1(\bmod 8)$ is more complicated and is discussed in [11, Section 24]. The following table lists the first few examples of square-free $d \in 2 \mathbb{N}$ with more than 2 distinct odd prime factors

\begin{tabular}{|c|c|c||c|c|c|}
\hline$d$ & $\varepsilon$ & $r \equiv 1 \bmod 4$ & $d$ & $\varepsilon$ & $r \equiv 3 \bmod 4$ \\
\hline 30 & $11+2 \sqrt{30}$ & 5 & 42 & $13+2 \sqrt{42}$ & 7 \\
\hline 66 & $65+8 \sqrt{66}$ & 33 & 78 & $53+6 \sqrt{78}$ & 3 \\
\hline 70 & $251+30 \sqrt{70}$ & 5 & 102 & $101+10 \sqrt{102}$ & 51 \\
\hline
\end{tabular}

\section{Calculations for $F=\mathbb{Q}(\sqrt{p})$ and $H=H_{\infty_{1}, \infty_{2}}$}

Let $p$ be a prime number, $F=\mathbb{Q}(\sqrt{p})$, and $H=H_{\infty_{1}, \infty_{2}}$ be the totally definite quaternion $F$-algebra that splits at all finite places of $F$. We calculate $h(G)=$ $h(H, G)$ for every finite group $G$. By [11, Corollary 18.4], $h(\mathbb{Q}(\sqrt{p}))$ is odd for every prime $p$. According to Proposition 1.1 (see also Remark 3.6 and [48, Section 3]),

$$
h(G)=h(F) t(G) \text { for all } G \text {. }
$$

Hence it is simpler to list all $t(G)$ instead. The case that $p \equiv 1(\bmod 4)$ with $p>5$ has already been treated by Hashimoto [18] using another method. We focus on the cases that $p \in\{2,3,5\}$ or $p \equiv 3(\bmod 4)$.

8.1. Case $p \in\{2,3,5\}$. Note that $N_{F / \mathbb{Q}}(\varepsilon)=-1$ if $p=2$ or 5 . If $p=3$, then $N_{F / \mathbb{Q}}(\varepsilon)=1,2 \varepsilon \in F^{\times 2}$, and $F(\sqrt{-1})=F(\sqrt{-3})$. The CM-extensions $K / F$ with $\left[O_{K}^{\times}: O_{F}^{\times}\right]>1$ are classified in [45, Section 2.8]. For a nontrivial element $\tilde{u} \in O_{K}^{\times} / O_{F}^{\times}$, we have

- $\operatorname{ord}(\tilde{u}) \in\{2,3,4\}$ if $p=2$;

- $\operatorname{ord}(\tilde{u}) \in\{2,3,4,6,12\}$ if $p=3$;

- $\operatorname{ord}(\tilde{u}) \in\{2,3,5\}$ if $p=5$.

The first four rows of Table 4.1 hold for $p=2,3,5$ as well. Thus our knowledge on minimal $D_{2}$ or $D_{3}$-orders applies here. If ord $(\tilde{u})=5$, then $p=5$ and $F(\tilde{u}) \simeq \mathbb{Q}\left(\zeta_{5}\right)$.

Let $H^{\prime}$ be an arbitrary totally definite quaternion $F$-algebra. We claim that $H^{\prime}=H_{\infty_{1}, \infty_{2}}$ if it contains an $O_{F}$-order $\mathcal{O}$ with noncyclic reduced unit group $\mathcal{O}^{\star}$. 
Indeed, we have

$$
\mathcal{O}^{\star} \in \begin{cases}\left\{D_{2}, D_{3}, D_{4}, A_{4}, S_{4}\right\} & \text { if } p=2 \\ \left\{D_{2}, D_{3}, D_{4}, A_{4}, S_{4}, D_{6}, D_{12}\right\} & \text { if } p=3 ; \\ \left\{D_{2}, D_{3}, A_{4}, D_{5}, A_{5}\right\} & \text { if } p=5\end{cases}
$$

First, suppose that $\mathcal{O}^{\star} \supseteq D_{2}$. Recall that a minimal $D_{2}$-order has discriminant $4 O_{F}$. Thus $H^{\prime}$ splits at all nondyadic primes of $F$. Since 2 is either inert or ramified in $F$ and the number of ramified places of $H^{\prime}$ is even, $H^{\prime}$ necessarily splits at the dyadic prime of $F$ as well. Similarly, $H^{\prime}=H_{\infty_{1}, \infty_{2}}$ if $\mathcal{O}^{\star} \supseteq D_{3}$. This verifies our claim in the cases $p=2,3$. Lastly, if $\mathcal{O}^{\star} \simeq D_{5}$ or $A_{5}$, then $\mathcal{O}$ contains a minimal $D_{5}$-order, which implies (by the proof of Proposition 4.3.5) that $H^{\prime}=\left\{\mathbb{Q}\left(\zeta_{5}\right),-1\right\}$. A direct calculation shows that $\left\{\mathbb{Q}\left(\zeta_{5}\right),-1\right\} \simeq H_{\infty_{1}, \infty_{2}}$.

Thanks to 38] (see also [44, Theorem 1.3]), we have

$$
t(H)=h(H)= \begin{cases}1 & \text { if } p=2,5 \\ 2 & \text { if } p=3 .\end{cases}
$$

Using the Magma Computational Algebra System [5], one easily checks that

\begin{tabular}{|c|c|c|c|}
\hline$p$ & 2 & 3 & 5 \\
\hline$t(G)$ & $t\left(S_{4}\right)=1$ & $t\left(S_{4}\right)=t\left(D_{12}\right)=1$ & $t\left(A_{5}\right)=1$ \\
\hline
\end{tabular}

This can also be obtained by hand using the mass formula (3.22), which we leave to the interested readers.

8.2. Case $p \equiv 3(\bmod 4)$ and $p>3$. First, we write down $t(G)$ for $G$ noncyclic. Since 3 is unramified in $F$, we have $3 \varepsilon \notin F^{\times 2}$. In particular, $t\left(D_{6}\right)=0$. On the other hand, $2 \varepsilon \in F^{\times 2}$ by [27, Lemma 3, p. 91] or [50, Lemma 3.2(1)]. Note that $H_{\infty_{1}, \infty_{2}} \simeq\left(\frac{-1,-1}{F}\right)$ since 2 is ramified in $F$. It follows from the results of Section 5 that

$$
t\left(S_{4}\right)=t\left(D_{4}\right)=1, \quad t\left(A_{4}\right)=t\left(D_{2}^{\dagger}\right)=0 .
$$

The unique conjugacy class of maximal orders with reduced unit group $S_{4}$ (resp. $\left.D_{4}\right)$ is represented by $\mathbb{O}_{24}$ in (5.8) (resp. $\mathbb{O}_{8}=\mathbb{O}_{4}^{\dagger}$ in (5.11)). Write $\varepsilon=a+b \sqrt{p}$ with $a, b \in \mathbb{N}$. Then $a$ is even and $b$ is odd by [50, Theorem 1.1(1)], and hence $\beth\left(\mathscr{O}_{2}^{\ddagger}\right)=2$ by Proposition 6.1.2. Therefore, $t\left(D_{2}^{\ddagger}\right)=0$ by Corollary 6.1.3. For $p>3, H_{\infty_{1}, \infty_{2}} \simeq\left(\frac{-1,-3}{F}\right)$ if and only if $p \equiv 2(\bmod 3)$. Thus

$$
t\left(D_{3}^{\dagger}\right)=\frac{1}{2}\left(1-\left(\frac{p}{3}\right)\right) \text {. }
$$

If $p \equiv 1(\bmod 3)$, then the fact that $2 \varepsilon \in F^{\times 2}$ implies that $\varepsilon \equiv-1\left(\bmod 3 O_{F}\right)$. Therefore, $\left(\frac{-\varepsilon,-3}{F}\right) \simeq H_{\infty_{1}, \infty_{2}}$ for all $p>3$ by Lemma 6.2.1. It follows from Corollary 6.2.3 that

$$
t\left(D_{3}^{\ddagger}\right)=\beth\left(\mathscr{O}_{3}^{\ddagger}\right)-t\left(S_{4}\right)-t\left(D_{6}\right)=\frac{1}{2}\left(1+\left(\frac{p}{3}\right)\right) .
$$

Combining (8.4) and (8.5), we obtain

$$
t\left(D_{3}\right)=t\left(D_{3}^{\dagger}\right)+t\left(D_{3}^{\dagger}\right)=1
$$

We pick $\mathbb{O}_{6}$ to be one of the maximal orders in the following table according to the conditions on $p$ so that $\llbracket \mathbb{O}_{6} \rrbracket$ is the unique member of $\operatorname{Tp}(H)$ with $\mathbb{O}_{6}^{\star} \simeq D_{3}$ : 


\begin{tabular}{|c|cc|c|}
\hline$p$ & \multicolumn{2}{|c|}{$\mathbb{O}_{6}$} & $\mathbb{O}_{6}^{\star}$ \\
\hline$p \equiv 11(\bmod 12)$ & $\mathbb{O}_{6}^{\dagger}$ & in $(5.22)$ & $D_{3}^{\dagger}$ \\
\hline$p \equiv 7(\bmod 24)$ & $\mathbb{O}$ & in $(6.38)$ & $D_{3}^{\ddagger}$ \\
\hline$p \equiv 19(\bmod 24)$ & $\mathbb{O}^{\prime}$ & in $(\underline{6.39})$ & $D_{3}^{\ddagger}$ \\
\hline
\end{tabular}

In summary, we have

$$
\operatorname{Tp}^{\natural}(H)=\left\{\llbracket \mathbb{O}_{24} \rrbracket, \llbracket \mathbb{O}_{8} \rrbracket, \llbracket \mathbb{O}_{6} \rrbracket\right\} .
$$

Next, we calculate $t\left(C_{n}\right)$ for $n \in\{2,3,4\}$. Recall that $\mathscr{B}_{n}$ denotes the finite set of $\mathrm{CM} O_{F}$-orders $B$ such that $B^{\times} / O_{F}^{\times} \simeq C_{n}$, and $\mathscr{B}:=\cup_{n>1} \mathscr{B}_{n}$. By the classification in Section $\left[7\right.$ the orders in $\mathscr{B}_{n}$ for $n \in\{2,3,4\}$ is listed in the following table:

\begin{tabular}{|c|c|c|c|}
\hline$n$ & 2 & 3 & 4 \\
\hline $\mathscr{B}_{n}$ & $O_{F}[\sqrt{-1}], \mathbb{O}_{F}[\sqrt{-\varepsilon}]$ & $O_{F(\sqrt{-3})}$ & $O_{F(\sqrt{-1})}, B_{1,2}$ \\
\hline
\end{tabular}

Here $B_{1,2} \subset F(\sqrt{-1})$ is the order defined in $(7.3)$, and $O_{F}[\sqrt{-\varepsilon}]$ coincides with the ring of integers of $F(\sqrt{-\varepsilon})=F(\sqrt{-2})$ by Lemma 7.2.5. For each $B \in \mathscr{B}_{n}$, we set

$$
t\left(C_{n}, B\right):=\#\left\{\llbracket \mathbb{O}^{\prime} \rrbracket \in \operatorname{Tp}(H) \mid \mathbb{O}^{\prime \star} \simeq C_{n} \text {, and } \operatorname{Emb}\left(B, \mathbb{O}^{\prime}\right) \neq \emptyset\right\} .
$$

It is shown in [48, Corollary 3.5] (see also Remark 3.6) that

$$
h\left(C_{n}, B\right)=h(F) t\left(C_{n}, B\right) \quad \forall B \in \mathscr{B}_{n} .
$$

The main tool to compute $h\left(C_{n}, B\right)$ (and in turn $t\left(C_{n}, B\right)$ ) is equation (3.19). In the current setting, we have $\mathfrak{d}(H)=O_{F}$ and hence $\omega(H)=0$. Moreover, $\mathcal{N}\left(\mathbb{O}^{\prime}\right)=$ $F^{\times} \mathbb{O}^{\prime \times}$ for every member $\llbracket \mathbb{O}^{\prime} \rrbracket \in \mathrm{Tp}^{\natural}(H)$. This can be checked individually for each $\llbracket \mathbb{O}^{\prime} \rrbracket \in \mathrm{Tp}^{\natural}(H)$ (see (4.26), (5.26), Proposition [5.6 and Section 6.2.5), or for all of $\mathrm{Tp}^{\natural}(H)$ at once by applying [48, Proposition 2.8]. The equation (3.19) simplifies as

$$
\sum_{\llbracket \mathbb{O}^{\prime} \rrbracket \in \mathrm{Tp}^{\natural}(H)} m\left(B, \mathbb{O}^{\prime}, \mathbb{O}^{\prime \times}\right)+2 t\left(C_{n}, B\right)=\frac{h(B)}{h(F)} .
$$

It remains to compute $m\left(B, \mathbb{O}^{\prime}, \mathbb{O}^{\prime \times}\right)$ for every $B \in \mathscr{B}$ and $\llbracket \mathbb{O}^{\prime} \rrbracket \in \mathrm{Tp}^{\natural}(H)$. We apply Proposition 3.4 to obtain the following table

TABLE 8.1. Values of $m\left(B, \mathbb{O}^{\prime}, \mathbb{O}^{\prime \times}\right)$ for $B \in \mathscr{B}$ and $\llbracket \mathbb{O}^{\prime} \rrbracket \in \mathrm{Tp}^{\natural}(H)$

\begin{tabular}{|c|c|c|c|c|c|}
\hline & $O_{F}[-1]$ & $O_{F}[\sqrt{-\varepsilon}]$ & $O_{F(\sqrt{-3})}$ & $B_{1,2}$ & $O_{F(\sqrt{-1})}$ \\
\hline $\mathbb{O}_{24}$ & 0 & 1 & 1 & 1 & 0 \\
\hline $\mathbb{O}_{8}$ & 1 & 1 & 0 & 0 & 1 \\
\hline $\mathbb{O}_{6}$ & $1-\left(\frac{p}{3}\right)$ & $1+\left(\frac{p}{3}\right)$ & 1 & 0 & 0 \\
\hline
\end{tabular}

Thanks to Proposition 3.4, it is enough to work out $B_{C}$ for each conjugacy class of maximal cyclic subgroups $C$ of $\mathbb{O}^{\prime \star}$. If $|C|=4$, then $B_{C} \simeq B_{1,2}$ for $\mathbb{O}^{\prime}=\mathbb{O}_{24}$, and $B_{C} \simeq O_{F(\sqrt{-1})}$ for $\mathbb{O}^{\prime}=\mathbb{O}_{8}$. If $|C|=2$ and $\mathbb{O}^{\prime}=\mathbb{O}_{24}$, then $B_{C} \simeq O_{F}[\sqrt{-\varepsilon}]$. We present $\mathbb{O}_{8}^{\star} \simeq D_{4}$ as in (4.7) with generators $\tilde{u}, \tilde{\eta} \in \mathbb{O}_{8}^{\star}$ satisfying the conditions of (4.8). Then $B_{\langle\tilde{u}\rangle} \simeq O_{F}[\sqrt{-1}]$ and $B_{\langle\tilde{u} \tilde{\eta}\rangle} \simeq O_{F}[\sqrt{-\varepsilon}]$. Lastly, if $|C|=2$ and $\mathbb{O}^{\prime}=\mathbb{O}_{6}$, then $B_{C} \simeq O_{F}[\sqrt{-1}]$ if $p \equiv 2(\bmod 3)$, and $B_{C} \simeq O_{F}[\sqrt{-\varepsilon}]$ if $p \equiv 1$ $(\bmod 3)$. 
Now we are ready to write down $t\left(C_{n}\right)$ for each $n \in\{2,3,4\}$. For simplicity, let $h(m):=h(\mathbb{Q}(\sqrt{m}))$ for any non-square $m \in \mathbb{Z}$. Combining (8.9) with Table 8.1, we obtain

$$
\begin{aligned}
t\left(C_{4}, O_{F(\sqrt{-1})}\right) & =\frac{h(F(\sqrt{-1}))}{2 h(F)}-\frac{1}{2}=\frac{1}{2}(h(-p)-1) ; \\
t\left(C_{4}, B_{1,2}\right) & =\frac{h\left(B_{1,2}\right)}{2 h(F)}-\frac{1}{2}=\frac{1}{2}\left(\left(2-\left(\frac{2}{p}\right)\right) h(-p)-1\right) .
\end{aligned}
$$

Here, we use (7.2) to rewrite $h(F(\sqrt{-1})) / h(F)$ (see also [45, (2.16)]). Then

$$
t\left(C_{4}\right)=t\left(C_{4}, O_{F(\sqrt{-1})}\right)+t\left(C_{4}, B_{1,2}\right)=\left(3-\left(\frac{2}{p}\right)\right) \frac{h(-p)}{2}-1 .
$$

Similarly, we have

$$
\begin{aligned}
t\left(C_{3}\right)= & t\left(C_{3}, O_{F(\sqrt{-3})}\right)=\frac{h(-3 p)}{4}-1 ; \\
& t\left(C_{2}, O_{F}[\sqrt{-1}]\right)=\left(2-\left(\frac{2}{p}\right)\right) \frac{h(-p)}{2}+\frac{1}{2}\left(\frac{p}{3}\right)-1 ; \\
& t\left(C_{2}, O_{F}[\sqrt{-\varepsilon}]\right)=\frac{h(-2 p)}{2}-\frac{1}{2}\left(\frac{p}{3}\right)-\frac{3}{2} ; \\
t\left(C_{2}\right)= & t\left(C_{2}, O_{F}[\sqrt{-1}]\right)+t\left(C_{2}, O_{F}[\sqrt{-\varepsilon}]\right) \\
= & \left(2-\left(\frac{2}{p}\right)\right) \frac{h(-p)}{2}+\frac{h(-2 p)}{2}-\frac{5}{2} .
\end{aligned}
$$

By the mass formula (3.22), we have for every maximal order $\mathbb{O}$ in $H$,

$$
\operatorname{Mass}(\mathbb{O})=\frac{1}{2} \zeta_{F}(-1) h(F) .
$$

Note that $\zeta_{F}(-1)>0$ by the Siegel's formula [49, Table 2, p. 70]. Combining (3.23) and (8.1), we obtain

$$
\begin{aligned}
t\left(C_{1}\right) & =\frac{\operatorname{Mass}(\mathbb{O})}{h(F)}-\frac{t\left(S_{4}\right)}{24}-\frac{t\left(D_{4}\right)}{8}-\frac{t\left(D_{3}\right)}{6}-\frac{t\left(C_{4}\right)}{4}-\frac{t\left(C_{3}\right)}{3}-\frac{t\left(C_{2}\right)}{2} \\
& =\frac{\zeta_{F}(-1)}{2}+\left(-7+3\left(\frac{2}{p}\right)\right) \frac{h(-p)}{8}-\frac{h(-2 p)}{4}-\frac{h(-3 p)}{12}+\frac{3}{2} .
\end{aligned}
$$

As $t(G)=0$ for all $G \notin\left\{S_{4}, D_{4}, D_{3}, C_{4}, C_{3}, C_{2}, C_{1}\right\}$, this concludes the computation of $t(G)$ for all $G$.

\section{Superspecial ABELIAN SURFACES}

We keep the notation of the previous section. In particular, $F=\mathbb{Q}(\sqrt{p})$, where $p$ is a prime number, and $H=H_{\infty_{1}, \infty_{2}}$. In this section, we give two applications of Theorems 1.5 and 1.6 to superspecial abelian surfaces. The first one gives for each finite group $G$ an explicit formula for the number of certain superspecial abelian surfaces with reduced automorphism group $G$; this one is straightforward. For the second application we construct superspecial abelian surfaces $X$ over some field $K$ of characteristic $p$ with endomorphism algebra $2 \operatorname{End}^{0}(X):=\operatorname{End}_{K}(X) \otimes \mathbb{Q} \simeq$

\footnotetext{
${ }^{2}$ For an abelian variety $X$ over a field $k$, we write $\operatorname{End}_{k}(X)$, or simply $\operatorname{End}(X)$ if the ground field $k$ is clear, for the endomorphism ring of $X$ over $k$. For any field extension $K / k$, we write $\operatorname{End}_{K}\left(X \otimes_{k} K\right)$ or simply $\operatorname{End}\left(X \otimes_{k} K\right)$ for the endomorphism ring of $X \otimes_{k} K$ (over $K$ ). Some
} 
$F$, provided that $p \not \equiv 1(\bmod 24)$. Recall that an abelian variety over a field $k$ of characteristic $p$ is said to be superspecial if it is isomorphic to a product of supersingular elliptic curves over an algebraic closure $\bar{k}$ of $k$.

9.1. The first application. Fix a Weil $p$-number $\pi=\sqrt{p}$ and a maximal order $\mathbb{O}$ in $H$. Let $\operatorname{Isog}_{F} O_{F}(\pi)$ denote the set of $\mathbb{F}_{p}$-isomorphism classes of simple abelian varieties $X$ over $\mathbb{F}_{p}$ with Frobenius endomorphism $\pi_{X}$ satisfying $\pi_{X}^{2}=p$ and with endomorphism ring $\operatorname{End}(X) \supset O_{F}$. Any member $X$ in $\operatorname{Isog}^{O_{F}}(\pi)$ is necessarily a superspecial abelian surface. Let

$$
\operatorname{Tp}(\pi):=\left\{\operatorname{End}(X) \mid X \in \operatorname{Isog}^{O_{F}}(\pi)\right\} / \simeq .
$$

By [44, Theorem 6.1.2], we have a natural bijection $\mathrm{Cl}(\mathbb{O}) \simeq \operatorname{Isog}^{O_{F}}(\pi)$. If the superspecial class $[X]$ corresponds to the ideal class $[I]$, then $\operatorname{End}(X) \simeq \mathcal{O}_{l}(I)$. Thus, one obtains a natural bijection $\operatorname{Tp}(\pi) \simeq \operatorname{Tp}(H)$. In particular,

$$
h(\pi):=\# \operatorname{Isog}^{O_{F}}(\pi)=h(H), \quad t(\pi):=\# \mathrm{Tp}^{O_{F}}(\pi)=t(H) .
$$

For any finite group $G$, define

$$
h(\pi, G):=\#\left\{X \in \operatorname{Isog}^{O_{F}}(\pi) \mid \operatorname{RAut}(X) \simeq G\right\},
$$

where $\operatorname{RAut}(X):=\operatorname{Aut}(X) / O_{F}^{\times}$is the reduced automorphism group of $X$. As the above correspondence preserves the automorphism groups, one has $h(\pi, G)=h(G)$, which is also equal to $h(F) t(G)$ by Proposition 1.1. By Theorems 1.5 and 1.6, we obtain explicit formulas for $h(\pi, G)$.

Proposition 9.1. For any finite group $G$, we have $h(\pi, G)=h(F) t(G)$, where an explicit formula for each $t(G)$ is given by Theorems 1.5 and 1.6.

9.2. Pop's result on embedding problems. We state a main result of F. Pop on embedding problems for large fields in [32. Let $k$ be any field. Let $\Gamma_{k}:=\operatorname{Gal}\left(k_{s} / k\right)$ denote the absolute Galois group of $k$, where $k_{s}$ is a separable closure of $k$. An embedding problem (EP) for $k$ is a diagram of surjective morphisms of profinite groups $\left(\gamma: \Gamma_{k} \rightarrow A, \alpha: B \rightarrow A\right)$. An EP is said to be finite if the profinite group $B$ is finite (hence $A$ is also finite); it is called split if the homomorphism $\alpha$ has a section. We write $k_{\mathrm{EP}}$ for the fixed subfield of $\operatorname{ker} \gamma$. A solution of an EP is a homomorphism of profinite groups $\beta: \Gamma_{k} \rightarrow B$ such that $\alpha \beta=\gamma$; it is called a proper solution if $\beta$ is surjective.

Let $K=k(t)$, where $t$ is a variable. We fix a separable closure $K_{s}$ of $K$ which contains $k_{s}$, and let $\pi: \Gamma_{K} \rightarrow \Gamma_{k}$ be the canonical projection. To each $\mathrm{EP}=(\gamma, \alpha)$ for $k$ one associates an $\mathrm{EP}_{K}:=(\gamma \pi, \alpha)$ for $K$. If $\beta$ is a solution of $\mathrm{EP}_{K}$, define

$$
K_{\beta}:=K_{s}^{\mathrm{ker} \beta}, \quad k_{\beta}:=K_{\beta} \cap k_{s} .
$$

A (proper) regular solution of an $\mathrm{EP}$ is a (proper) solution $\beta$ of $\mathrm{EP}_{K}$ such that $k_{\beta}=k_{\mathrm{EP}}$.

The regular inverse Galois problem for $k$ asks whether for a given finite group $G$, there exists a regular finite Galois extension $L / k(t)$ (regularity means that $L \cap k_{s}=k$ in a separable closure $k(t)_{s}$ of $\left.k(t)\right)$ with Galois group $\operatorname{Gal}(L / k(t)) \simeq G$. If $A=\{1\}$ and $B=G$ is finite, then a proper solution for an EP is precisely a solution for

authors denote the latter by $\operatorname{End}_{K}(X)$. We caution the reader that the notation $\operatorname{End}(X)$ is also used for the endomorphism ring of $X \otimes_{k} \bar{k}$ over $\bar{k}$ in the literature, where $\bar{k}$ is an algebraic closure of $k$. 
an inverse Galois problem, and a proper regular solution for an EP is precisely a solution for a regular inverse Galois problem.

Definition 9.1. A field $k$ is said to be large if for any smooth curve $C$ over $k$, we have implication

$$
C(k) \neq \emptyset \Longrightarrow \# C(k)=\infty .
$$

Theorem 9.2 ( [32, Main Theorem A]). Assume that $k$ is large. Then every finite split EP for $k$ has proper regular solutions. In particular, every finite group $G$ is regularly realizable as a Galois group over $k(t)$.

The proof in 32 also shows that that there are infinitely many solutions in Theorem 9.2 .

9.3. The second application. Let $X_{0}$ be an abelian variety over any field $K$. It is well-known that the Galois cohomology $H^{1}\left(\Gamma_{K}\right.$, Aut $\left.\left(X_{0} \otimes_{K} K_{s}\right)\right)$ classifies all $K$-forms of $X_{0} / K$ up to $K$-isomorphism. Any class in $H^{1}\left(\Gamma_{K}\right.$, Aut $\left.\left(X_{0} \otimes_{K} K_{s}\right)\right)$ is represented by a 1 -cocycle $\xi=\left(\xi_{\sigma}\right) \in Z^{1}\left(\operatorname{Gal}(L / K), \operatorname{Aut}\left(X_{0} \otimes_{K} L\right)\right)$ for some finite Galois extension $L / K$.

Lemma 9.1. Suppose $X_{\xi} / K$ is the abelian variety corresponding to a 1-cocycle $\xi=\left(\xi_{\sigma}\right) \in Z^{1}\left(\operatorname{Gal}(L / K)\right.$, Aut $\left.\left(X_{0} \otimes_{K} L\right)\right)$. Then

$$
\operatorname{End}\left(X_{\xi}\right) \simeq\left\{y \in \operatorname{End}\left(X_{0} \otimes_{K} L\right) \mid \xi_{\sigma} \sigma(y) \xi_{\sigma}^{-1}=y, \forall \sigma \in \operatorname{Gal}(L / K)\right\} .
$$

Lemma 9.2. For any fixed power $q$ of $p$ and any positive integer $\ell$, the field

$$
k:=\mathbb{F}_{q^{\ell \infty}}:=\bigcup_{m \geq 1} \mathbb{F}_{q^{\ell^{m}}}
$$

is large.

Proof. This follows immediately from the Hasse-Weil bound for the size $\# C\left(\mathbb{F}_{q^{\ell m}}\right)$ of $\mathbb{F}_{q^{e^{m}}}$-rational points of a curve $C$.

Proposition 9.3. There exists a maximal $O_{F}$-order $\mathbb{O}$ in $H=H_{\infty_{1}, \infty_{2}}$ for which the unit group $\mathbb{O}^{\times}$contains a finite non-abelian group if and only if $p \not \equiv 1(\bmod 24)$.

Proof. The reduced norm map Nr : $\mathbb{O}^{\times} \rightarrow O_{F}^{\times}$induces a map $\mathrm{Nr}: \mathbb{O}^{\star} \rightarrow O_{F,+}^{\times} /\left(O_{F}^{\times}\right)^{2}$. The kernel of this map is $\mathbb{O}^{1} /\{ \pm 1\}$, where $\mathbb{O}^{1} \subset \mathbb{O}^{\times}$is the reduced norm one subgroup. Thus the index $\left[\mathbb{O}^{\star}: \mathbb{O}^{1} /\{ \pm 1\}\right] \in\{1,2\}$, and $\left[\mathbb{O}^{\star}: \mathbb{O}^{1} /\{ \pm 1\}\right]=1$ if $N(\varepsilon)=-1$. If $p \leq 5$ or $p \equiv 3(\bmod 4)$. then there is a maximal order $\mathbb{O}$ such that $\mathbb{O}^{\star} \simeq S_{4}$ or $A_{5}$ by Theorem [1.6. Then the finite group $\mathbb{O}^{1} /\{ \pm 1\}$ must be non-abelian and hence $\mathbb{O}^{1}$ is a non-abelian finite subgroup of $\mathbb{O}^{\times}$. Note that $p \leq 5$ or $p \equiv 3(\bmod 4)$ implies that $p \not \equiv 1(\bmod 24)$. Now assume that $p \equiv 1(\bmod 4)$ and $p \geq 7$. In this case $N(\varepsilon)=-1$ by [11, Corollary 18.4bis] and $\mathbb{O}^{\star}=\mathbb{O}^{1} /\{ \pm 1\}$. One has $\mathbb{O}^{1} /\{ \pm 1\} \simeq C_{n}$ for $n=1,2,3$ if and only if $\mathbb{O}^{1}=\mu_{2 n} \simeq C_{2 n}$. It then follows from Theorem 1.5 that there exists a maximal order $\mathbb{O}$ with $\mathbb{O}^{1}$ non-abelian if and only if $\left(\frac{2}{p}\right)=-1$ or $\left(\frac{p}{3}\right)=-1$. The latter is equivalent to $p \not \equiv 1(\bmod 24)$ under the condition $p \equiv 1(\bmod 4)$. This proves the proposition.

Proposition 9.4. Suppose $X_{0} / \mathbb{F}_{p} \in \operatorname{Isog}^{O_{F}}(\pi)$ is a member such that $\operatorname{Aut}\left(X_{0}\right)$ contains a finite non-abelian group $G$. There exist a positivie integer $\ell$ and infinitely many abelian varieties $X$ over $K:=\mathbb{F}_{p^{e \infty}}(t)$, which are $K$-forms of $X_{0} \otimes_{\mathbb{F}_{p}} K$, such that the endomorphism algebra $\operatorname{End}^{0}(X)$ of $X$ is isomorphic to $F=\mathbb{Q}(\sqrt{p})$. 
Proof. Let $\mathbb{F}_{p^{n}} / \mathbb{F}_{p}$ be a finite extension such that $\operatorname{End}\left(X_{0} \otimes \overline{\mathbb{F}}_{p}\right)=\operatorname{End}\left(X_{0} \otimes \mathbb{F}_{p^{n}}\right)$, where we omit the subscript $\mathbb{F}_{p}$ from the scalar extensions of $X_{0}$. Let $\ell$ be any positive integer with $(\ell, n)=1$ and put $k=\mathbb{F}_{p^{\ell \infty}}$ and $K=k(t)$. Then $k \cap \mathbb{F}_{p^{n}}=\mathbb{F}_{p}$ and one has $\operatorname{End}\left(X_{0} \otimes k\right)=\operatorname{End}\left(X_{0}\right)$, which is a maximal order in $H$. Since $K / k$ is primary, $\operatorname{End}\left(X_{0} \otimes K\right)=\operatorname{End}\left(X_{0} \otimes k\right)=\operatorname{End}\left(X_{0}\right)$ by Chow's Theorem [10; see also [12, Theorem 3.19]. Since $k$ is large (Lemma 9.2), there exist infinitely many regular finite Galois extensions $L / K$ with Galois group $\operatorname{Gal}(L / K) \simeq G$ by Theorem 9.2, Consider the homomorphism $\xi: \operatorname{Gal}(L / K) \rightarrow \operatorname{Aut}\left(X_{0} \otimes L\right)$ defined by the composition

$$
\operatorname{Gal}(L / K) \stackrel{\sim}{\longrightarrow} G \subset \operatorname{Aut}\left(X_{0}\right) \subset \operatorname{Aut}\left(X_{0} \otimes L\right) .
$$

Since $L / K$ is regular, we have $\operatorname{End}\left(X_{0} \otimes L\right)=\operatorname{End}\left(X_{0} \otimes K\right)=\operatorname{End}\left(X_{0}\right)$ by Chow's Theorem again. Thus, $\operatorname{Gal}(L / K)$ acts trivially on $\operatorname{Aut}\left(X_{0} \otimes L\right)$ and hence $\xi$ is a 1-cocyle. Let $X / K$ be the abelian variety corresponding to $\xi$. Then by Lemma 9.1 $\operatorname{End}^{0}(X)$ is isomorphic to the centralizer of $G$ in $\operatorname{End}^{0}\left(X_{0}\right)=H$ which is the center $F=\mathbb{Q}(\sqrt{p})$.

Corollary 9.5. Assume that $p \not \equiv 1(\bmod 24)$. Then there is a superspecial abelian surface $X$ over some field $K$ of characteristic $p$ such that $\operatorname{End}_{K}^{0}(X) \simeq \mathbb{Q}(\sqrt{p})$.

Proof. This follows from Propositions 9.3 and 9.4

\section{ACKNOWLEDGEMENTS}

J. Xue is partially supported by the Natural Science Foundation of China grant \#11601395. He thanks the Morningside Center of Mathematics (CAS), Academia Sinica and NCTS for their hospitality and great working conditions during his visits in 2017, hosted by Xu Shen and Chia-Fu Yu, respectively. C.-F. Yu is partially supported by the grants MoST 104-2115-M-001-001-MY3 and 107-2115-M-001-001MY2. The authors thank the referees for careful readings and helpful suggestions that have improved the exposition of the paper significantly.

\section{REFERENCES}

[1] Şaban Alaca and Kenneth S. Williams. Introductory algebraic number theory. Cambridge University Press, Cambridge, 2004.

[2] Roger C. Alperin. Remarks on a problem of Eisenstein. JP J. Algebra Number Theory Appl., $7(1): 97-102,2007$.

[3] Luis Arenas-Carmona. Eichler orders, trees and representation fields. Int. J. Number Theory, 9(7):1725-1741, 2013.

[4] Luis Arenas-Carmona and Ignacio Saavedra. On some branches of the Bruhat-Tits tree. Int. J. Number Theory, 12(3):813-831, 2016.

[5] Wieb Bosma, John Cannon, and Catherine Playoust. The Magma algebra system. I. The user language. J. Symbolic Comput., 24(3-4):235-265, 1997. Computational algebra and number theory (London, 1993).

[6] Juliusz Brzezinski. On orders in quaternion algebras. Comm. Algebra, 11(5):501-522, 1983.

[7] Juliusz Brzezinski. On automorphisms of quaternion orders. J. Reine Angew. Math., 403:166$186,1990$.

[8] Juliusz Brzezinski. Definite quaternion orders of class number one. J. Théor. Nombres Bordeaux, 7(1):93-96, 1995. Les Dix-huitièmes Journées Arithmétiques (Bordeaux, 1993).

[9] D. A. Buell, H. C. Williams, and K. S. Williams. On the imaginary bicyclic biquadratic fields with class-number 2. Math. Comp., 31(140):1034-1042, 1977.

[10] Wei-Liang Chow. Abelian varieties over function fields. Trans. Amer. Math. Soc., 78:253-275, 1955. 
[11] P. E. Conner and J. Hurrelbrink. Class number parity, volume 8 of Series in Pure Mathematics. World Scientific Publishing Co., Singapore, 1988.

[12] Brian Conrad. Chow's $K / k$-image and $K / k$-trace, and the Lang-Néron theorem. Enseign. Math. (2), 52(1-2):37-108, 2006.

[13] Charles W. Curtis and Irving Reiner. Methods of representation theory. Vol. II. Pure and Applied Mathematics (New York). John Wiley \& Sons, Inc., New York, 1987. With applications to finite groups and orders, A Wiley-Interscience Publication.

[14] Yurij A. Drozd and Vladimir V. Kirichenko. Finite-dimensional algebras. Springer-Verlag, Berlin, 1994. Translated from the 1980 Russian original and with an appendix by Vlastimil Dlab.

[15] Martin Eichler. Zur Zahlentheorie der Quaternionen-Algebren. J. Reine Angew. Math., 195:127-151 (1956), 1955.

[16] A. Fröhlich and M. J. Taylor. Algebraic number theory, volume 27 of Cambridge Studies in Advanced Mathematics. Cambridge University Press, Cambridge, 1993.

[17] Emmanuel Hallouin and Christian Maire. Cancellation in totally definite quaternion algebras. J. Reine Angew. Math., 595:189-213, 2006.

[18] Ki-ichiro Hashimoto. Twisted trace formula of the Brandt matrix. Proc. Japan Acad. Ser. A Math. Sci., 53(3):98-102, 1977.

[19] G. Herglotz. Über einen Dirichletschen Satz. Math. Z., 12(1):255-261, 1922.

[20] Jae Moon Kim and Jado Ryu. On the class number and the fundamental unit of the real quadratic field $k=\mathbb{Q}(\sqrt{p q})$. Bull. Aust. Math. Soc., 85(3):359-370, 2012.

[21] Markus Kirschmer and David Lorch. Ternary quadratic forms over number fields with small class number. J. Number Theory, 161:343-361, 2016.

[22] Markus Kirschmer and John Voight. Algorithmic enumeration of ideal classes for quaternion orders. SIAM J. Comput., 39(5):1714-1747, 2010.

[23] Yoshiyuki Kitaoka. Quaternary even positive definite quadratic forms of prime discriminant. Nagoya Math. J., 52:147-161, 1973.

[24] Ke-Zheng Li and Frans Oort. Moduli of supersingular abelian varieties, volume 1680 of Lecture Notes in Mathematics. Springer-Verlag, Berlin, 1998.

[25] Colin Maclachlan and Alan W. Reid. The arithmetic of hyperbolic 3-manifolds, volume 219 of Graduate Texts in Mathematics. Springer-Verlag, New York, 2003.

[26] Daniel A. Marcus. Number fields. Springer-Verlag, New York, 1977. Universitext.

[27] Thomas M. McCall, Charles J. Parry, and Ramona Ranalli. Imaginary bicyclic biquadratic fields with cyclic 2-class group. J. Number Theory, 53(1):88-99, 1995.

[28] O. Timothy O'Meara. Introduction to quadratic forms. Classics in Mathematics. SpringerVerlag, Berlin, 2000. Reprint of the 1973 edition.

[29] Arnold Pizer. On the arithmetic of quaternion algebras. Acta Arith., 31(1):61-89, 1976.

[30] Arnold K. Pizer. Type numbers of Eichler orders. J. Reine Angew. Math., 264:76-102, 1973.

[31] Paul Ponomarev. Class number formulas for quaternary quadratic forms. Acta Arith., 39(1):95-104, 1981.

[32] Florian Pop. Embedding problems over large fields. Ann. of Math. (2), 144(1):1-34, 1996.

[33] I. Reiner. Maximal orders, volume 28 of London Mathematical Society Monographs. New Series. The Clarendon Press Oxford University Press, Oxford, 2003. Corrected reprint of the 1975 original, With a foreword by M. J. Taylor.

[34] Jean-Pierre Serre. Local fields, volume 67 of Graduate Texts in Mathematics. Springer-Verlag, New York, 1979. Translated from the French by Marvin Jay Greenberg.

[35] Daniel Smertnig. A note on cancellation in totally definite quaternion algebras. J. Reine Angew. Math., 707:209-216, 2015.

[36] Peter Stevenhagen. On a problem of Eisenstein. Acta Arith., 74(3):259-268, 1996.

[37] Fang-Ting Tu. On orders of $M(2, K)$ over a non-Archimedean local field. Int. J. Number Theory, 7(5):1137-1149, 2011.

[38] Marie-France Vignéras. Nombre de classes d'un ordre d'Eichler et valeur au point -1 de la fonction zêta d'un corps quadratique réel. Enseignement Math. (2), 21(1):69-105, 1975.

[39] Marie-France Vignéras. Simplification pour les ordres des corps de quaternions totalement définis. J. Reine Angew. Math., 286/287:257-277, 1976.

[40] Marie-France Vignéras. Arithmétique des algèbres de quaternions, volume 800 of Lecture Notes in Mathematics. Springer, Berlin, 1980. 
[41] Lawrence C. Washington. Introduction to cyclotomic fields, volume 83 of Graduate Texts in Mathematics. Springer-Verlag, New York, second edition, 1997.

[42] Fu-Tsun Wei and Chia-Fu Yu. Class numbers of central simple algebras over global function fields. Int. Math. Res. Not. IMRN, (11):3525-3575, 2015.

[43] Robert A. Wilson. The finite simple groups, volume 251 of Graduate Texts in Mathematics. Springer-Verlag London, Ltd., London, 2009.

[44] Jiangwei Xue, Tse-Chung Yang, and Chia-Fu Yu. Supersingular abelian surfaces and Eichler's class number formula. ArXiv e-prints, April 2014, arXiv:1404.2978 to appear in The Asian Journal of Mathematics.

[45] Jiangwei Xue, Tse-Chung Yang, and Chia-Fu Yu. Numerical invariants of totally imaginary quadratic $\mathbb{Z}[\sqrt{p}]$-orders. Taiwanese J. Math., 20(4):723-741, 2016.

[46] Jiangwei Xue, Tse-Chung Yang, and Chia-Fu Yu. On superspecial abelian surfaces over finite fields. Doc. Math., 21:1607-1643, 2016.

[47] Jiangwei Xue, Tse-Chung Yang, and Chia-Fu Yu. On superspecial abelian surfaces over finite fields II. ArXiv e-prints, January 2018, arXiv:1801.09374

[48] Jiangwei Xue and Chia-Fu Yu. On superspecial abelian surfaces and type numbers of totally definite quaternion algebras. arXiv e-prints, September 2018, arXiv:1809.04316

[49] Don Zagier. On the values at negative integers of the zeta-function of a real quadratic field. Enseignement Math. (2), 22(1-2):55-95, 1976.

[50] Zhe Zhang and Qin Yue. Fundamental units of real quadratic fields of odd class number. $J$. Number Theory, 137:122-129, 2014.

(Li) School of Mathematics and Statistics, Wuhan University, Luojiashan, Wuhan, Hubei, 430072, P.R. China.

E-mail address: qun_1@whu.edu.cn

(Xue) Collaborative Innovation Centre of Mathematics, School of Mathematics and Statistics, Wuhan University, Luojiashan, Wuhan, Hubei, 430072, P.R. China.

(Xue) Hubei Key Laboratory of Computational Science (Wuhan University), Wuhan, Hubei, 430072, P.R. China.

E-mail address: xue_j@whu.edu.cn

(Yu) Institute of Mathematics, Academia Sinica, Astronomy-Mathematics Building, No. 1, Sec. 4, Roosevelt Road, TAipei 10617, TAIWAN.

(Yu) National Center for Theoretical Sciences, Astronomy-Mathematics Building, No. 1, Sec. 4, Roosevelt Road, Taipei 10617, TAIWAN.

E-mail address: chiafu@math.sinica.edu.tw 